DEPARTMENT OF THE INTERIOR

U.S. GEOLOGICAL SURVEY

Gravity observations by the U.S. Geological Survey in northwest Nevada, southeast Oregon, and northeast California, 1984-1986

by

Donald Plouff ${ }^{1}$

Open-File Report 87-639

This report is preliminary and has not been reviewed for conformity with U.S. Geological Survey editorial standards. Any use of trade names is for descriptive purposes only and does not imply endorsement by the U.S. Geological survey.

${ }^{1}$ Menlo Park, California 
A total of 1,020 gravity stations were established in parts of northwest Nevada, southeast oregon, and northeast California by the U.S. Geological Survey during 1984 to 1986 ( $\mathrm{fig}$. 1). The purpose of the gravity survey was to provide a regional geophysical background for evaluation of mineral resources in public lands (for example, Keith and others, 1986; Noble and others, 1987a,b; Sorensen and others, 1987; and Vander Meulen and others, 1987). This survey also extends previous surveys near Adel, Oreg. (Plouff and Conradi, 1975), McDermitt, Nev. (Plouff, 1976; 1977a), and the Sheldon Antelope Range, Nev. (Plouff, 1984).

\section{VALUES OF OBSERVED GRAVITY}

The datum of observed gravity for stations listed in this report is the International Gravity Standardization Net of 1971 (IGSN-71) described by Morelli (1974). Ties were made to five base stations merged with the IGSN-71 datum by the Defense Mapping Agency (Jablonski, 1974). These stations are located in or near Denio, Gerlach, McDermitt, and Winnemucca, Nev. and Jordan Valley, Oreg. Ties were made to two base stations described by Chapman (1966) in Cedarville and Susanville, Calif. Chapman's values of observed gravity, which were based on the datum of Woollard and Rose (1963), were converted to the IGSN-71 datum by subtracting constants of 14.51 and 14.49 milligals (mGal), respectively, as derived from oliver and others (1980). The accuracy of this conversion is about $0.05 \mathrm{mGal}$. All base stations used in this report are described in Table 1.

Tide-corrected drift curves were prepared for all the observed gravity data (fig. 2). The curves include the effect of uncertainty in evaluating the observed gravity for base and repeated stations that form the drift curves as well as instrumental drift. The range of daily drift seldom exceeded 0.1 mGal. Drift was markedly higher during two days in which helicopter transportation was used. Helicopter transportation was used on June 24 (0.11 mGal) and October 18, 1984 and August $17(0.23 \mathrm{mGal})$, August 18, and August $21,1985$.

\section{CALCULATION OF GRAVITY ANOMALIES}

Free-air gravity anomalies were determined by using the Geodetic Reference System 1967 formula (GRS-67) for the normal gravity on the ellipsoid (International Association of Geodesy, 1971, p. 60) and Swick's (1942, p.65) formula for the free-air correction. Bouguer, Earth's curvature, and terrain corrections were added to the free-air gravity anomaly at each station to determine complete Bouguer anomalies at a standard reduction density of 2.67 $\mathrm{g} / \mathrm{cm}^{3}$. Terrain corrections were determined to a distance of $0.89 \mathrm{~km}$ from each station by using Hammer's (1939) method of esimating elevations in cylindrical sectors around the stations. Terrain corrections in the remaining distance interval to $166.7 \mathrm{~km}$ were determined with a computer program that uses topography digitized at intervals of 15 seconds, 1 minute, and 3 minutes (Plouff, 1977b). Isostatic corrections were estimated by using a computer program by Jachens and Roberts (1981). That program calculates the effect of Airy-Heiskanen isostatic compensation by using 3-minute topographic digitization. The parameters assumed for the isostatic model were $25 \mathrm{~km}$ for the normal crustal thickness, $2.67 \mathrm{~g} / \mathrm{cm}^{3}$ for the density of the crust, and a density contrast of $0.4 \mathrm{~g} / \mathrm{cm}^{3}$ between the lower crust and the upper mantle. Isostatic corrections were subtracted from values of the Bouguer gravity anomaly to obtain values of isostatic residual gravity. 

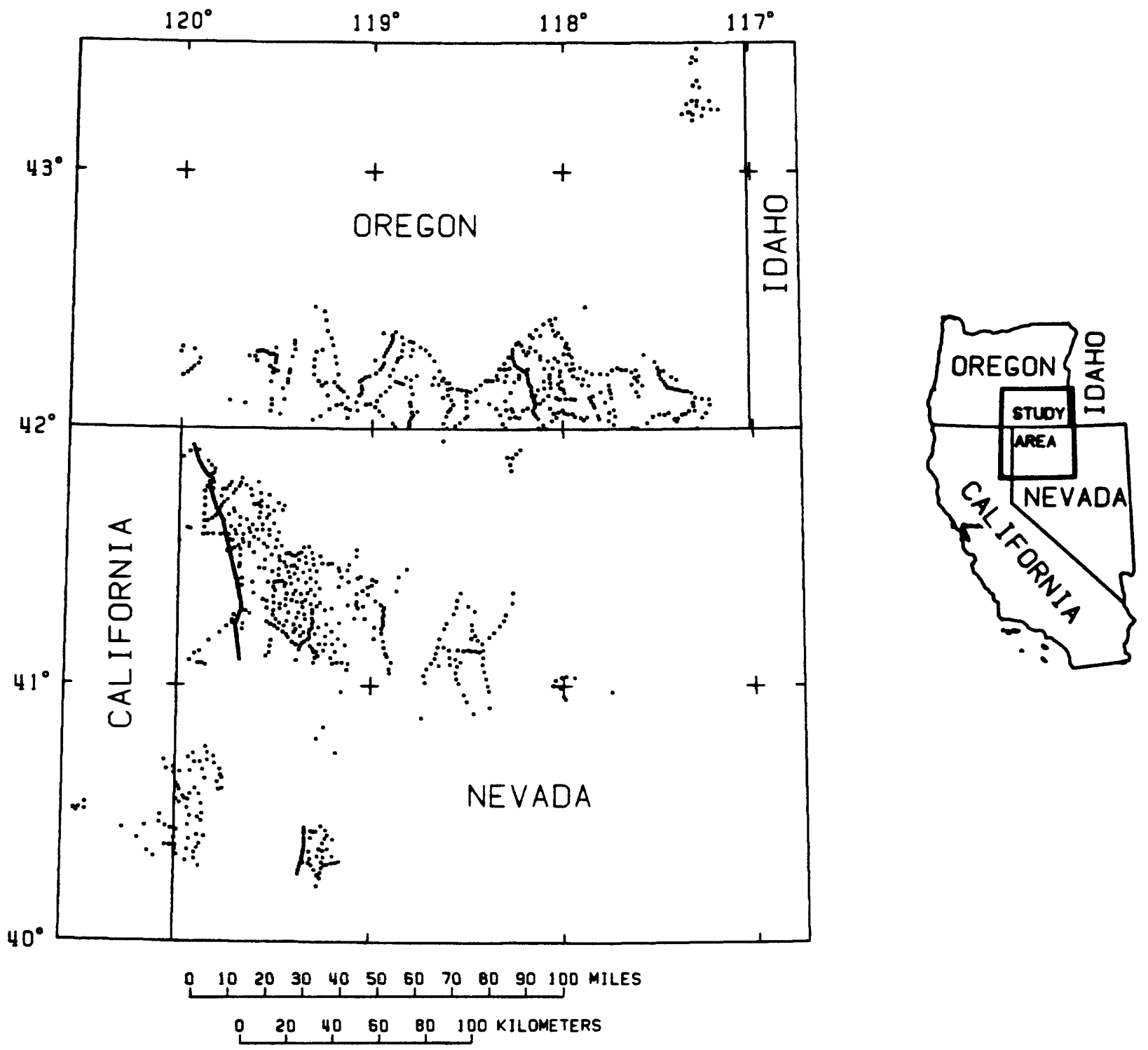

Figure 1. Locations of gravity stations. 

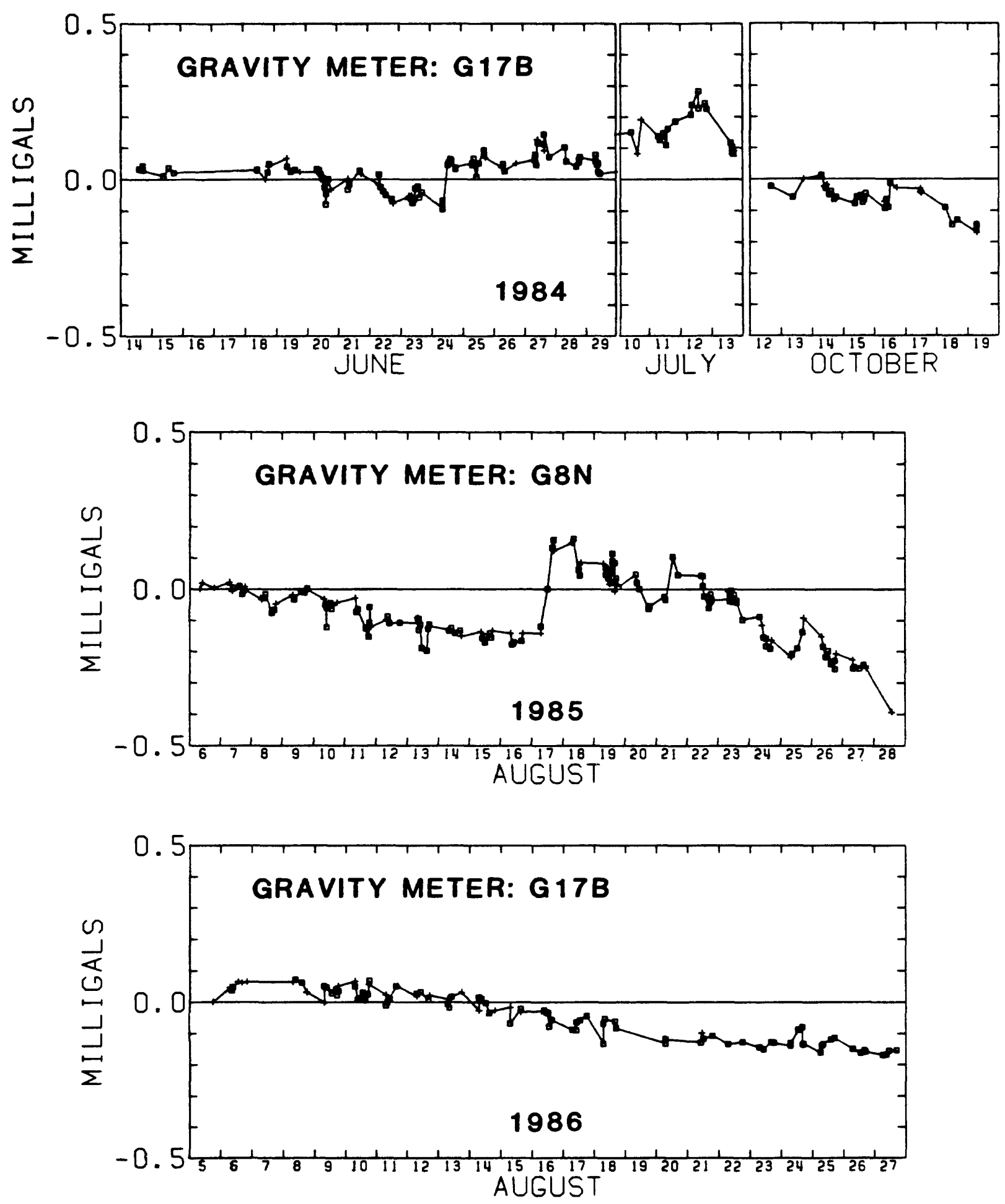

Figure 2. Drift curves for gravity readings. Points that delineate drift curves are determined from observations at base stations or at stations with repeated readings. Lines are loci of linearly interpolated values of drift. Square, repeat station. Plus sign, base station. 


\section{LISTS OF PRINCIPAL FACTS}

Tables 6, 7, and 8 list principal facts for the gravity data. The information listed for each station fits in 80 columns on a line in a computer file and is reformatted for readability in the tables. Station names consist of 8 or less characters. Locations are described in geographic coordinates expressed in degrees and minutes. Inasmuch as most topographic maps and benchmark lists in the area indicate elevations in feet, elevations are expressed in feet.

Values of observed gravity are referenced to the IGSN-71 datum and are expressed in milligals. The free-air gravity anomaly, expressed in units of milligals, uses the GRS-67 formula for normal gravity on the spheroid and includes higher order terms of Swick's (1942, p. 65) formula for the free-air correction. Terrain corrections are subdivided into two columns of values expressed in milligals. The first column is the hand terrain correction determined through Hammer's (1939) F-ring. The second column is the part calculated from digital topography between Hammer's (1939) F-ring (0.89 km) and $166.7 \mathrm{~km}$. Bouguer gravity anomalies ("BOUG ANOM") and values of the isostatic residual gravity ("ISOS ANOM") are expressed in milligals.

"ACC CODE" refers to a 4-digit accuracy code. The first digit describes the location and the type of elevation at the station (table 2). The second digit provides an estimate of the elevation accuracy (table 3 ), which relates to a large source of error $(0.06 \mathrm{mGal} / \mathrm{ft})$ when calculating the Bouguer gravity anomaly. The third digit indicates the accuracy of horizontal location (table 4). The fourth digit indicates the accuracy of observed gravity (table 5). This code depends on the type of gravity meter used, the magnitude of drift, and the number and quality of repeat readings. The "DATE" is the most recent date, expressed in month, day, and year (without the leading 19), when gravity was observed at the station. "BASE NAME" is the 5-digit name of the base station to which the station was tied.

The following statistical information summarizes the principal facts for the 1,020 gravity stations. Elevations, which range from 3,598 to $8,041 \mathrm{ft}$ above sea level, have an average elevation of $5375 \pm 802 \mathrm{ft}$, where the last number indicates the standard deviation. Values of observed gravity range from 979,623 to $980,125 \mathrm{mGal}$ with an average of $979,822 \pm 73 \mathrm{mGal}$. Free-air gravity anomalies range from -47 to $93 \mathrm{mGal}$ with an average of $13 \pm 24 \mathrm{mGal}$. Hand terrain corrections to a distance of $0.89 \mathrm{~km}$ from each station range from 0.0 to $6.7 \mathrm{mGal}$ with an average of $0.3+0.6 \mathrm{mGal}$. Total terrain corrections range from 0.1 to $19.4 \mathrm{mGal}$ with an average of $1.7 \pm 2.0 \mathrm{mGal}$. Bouguer gravity anomalies range from -200 to $-121 \mathrm{mGal}$ with an average of $-170 \pm 15 \mathrm{mGal}$. Values of isostatic residual gravity range from -31 to 25 mGal with an average of $-7 \pm 10 \mathrm{mGal}$. The standard deviation, which reflects scatter of data, is $5 \mathrm{mGal}$ less for values of isostatic residual gravity compared to values of the Bouguer gravity anomaly.

\section{ACKNOWLEDGMENTS}

C.F. Erdman, John Mariano, J.H. Laudon, V.E. Langenheim, D.R. Kietzman, and J.J. Rytuba of the U.S. Geological Survey assisted the fieldwork. J.J. Rytuba suggested an extension of the work to nearby areas of mapped calderas in Oregon. The Los Angeles Department of Water and Power supplied index maps and elevations at power poles along the Sylmar-oregon high-voltage DC line (A.A. 
Galindo, written commun., 1985). Personnel of the Bureau of Land Management at Cedarville, Calif., supplied helpful information on the status of roads and coordinated the use of a helicopter assigned to their office. M.M. Hamilton of the U.S. Bureau of Mines shared trailer facilities and helicopter usage.

\section{REFERENCES}

Chapman, R.H., 1966, The California Division of Mines and Geology gravity base station network: California Division of Mines and Geology Special Report 90 and Supplement, $53 \mathrm{p}$.

Hammer, Sigmund, 1939, Terrain corrections for gravimeter stations: Geophysics, v. 4, no. 3, p. 184-194.

International Association of Geodesy, 1971, Geodetic reference system 1967: International Association of Geodesy Special Publication no. 3, $116 \mathrm{p}$.

Jablonski, H.M., 1974, World relative gravity network North America, Parts 1 and 2: Defense Mapping Agency Aerospace Center Reference Publication no. 25, originally published 1970, revised 1974 with supplement updating gravity values to the IGSN-71 datum, $1635 \mathrm{p}$.

Jachens, R.C., and Roberts, C.W., 1981, Documentation of a FORTRAN program, "isocomp", for computing isostatic residual gravity: U.S. Geological Survey Open-File Report 81-574, 26 p.

Keith, W.J., Turner, R.L., Plouff, Donald, and Rumsey, C.M., 1986, Mineral resources of the Mount Limbo Wilderness Study Area, Pershing County Nevada: U.S. Geological Survey Bulletin 1726-A, 11 p.

Morelli, C., (Ed.), 1974, The International gravity standardization net 1971: International Association of Geodesy Special Publication no. 4, $194 \mathrm{p}$.

Noble, D.C., Plouff, Donald, Bergquist, J.R., Barton, H.N., and Olson, J.E., 1987a, Mineral resources of the Pahute Peak Wilderness Study Area, Humboldt County, Nevada: U.S. Geological Survey Bulletin 1726-C, 12 p.

Noble, D.C., Plouff, Donald, Bergquist, J.R., Neumann, T.R., and Close, T.J., 1987b, Mineral resources of the High Rock Lake Wilderness Study Area, Humboldt County, Nevada: U.S. Geological Survey Bulletin 1707-A, 9 p.

Oliver, H.W., Robbins, S.L., and Chapman, R.H., 1980, Gravity measurements, reductions, and conversion formulas to IGSN 71 and GRS 67, appendix 1 in Oliver, H.W., ed., Interpretation of the gravity maps of California and its continental margin: California Division of Mines and Geology Bulletin 205, 47-52 p.

Plouff, Donald, 1976, Principal facts for gravity observations near McDermitt, Nevada: U.S. Geological Survey Open-File Report 76-599, 21 p.

----1977a, Gravity observations near McDermitt, Nevada during 1976: U.S. Geological Survey Open-File Report 77-536, $13 \mathrm{p}$.

---1977b, Preliminary documentation for a FORTRAN program to compute gravity terrain corrections based on topography digitized on a geographic grid: U.S. Geological Survey Open-File Report 77-535, 45 p.

---1984, Interpretation of aeromagnetic and gravity data, Charles Sheldon Wilderness study area, Nevada and Oregon, Chapter B, in Mineral resources of the Charles Sheldon wilderness study area, Humboldt and Washoe Counties, Nevada and Lake and Harney Counties, Oregon: U.S. Geological Survey Bulletin 1538, p. 35-49, 1 pl.

Plouff, Donald, and Conradi, Arthur, Jr., 1975, Gravity and magnetic profiles and maps, Crump Geyser area, Oregon: U.S. Geological Survey Open-File Report 75-346, 2 p., 12 sheets. 
Sorensen, M.L., Plouff, Donald, Turner, R.L., and Hamilton, M.M., 1987, Mineral resources of the South Jackson Mountains wilderness Study Area, Humboldt County, Nefada: U.S. Geological Survey Bulletin 1726-B, $14 \mathrm{p}$. Swick, C.H., 1942, Pendulum gravity measurements and isostatic reductions: U.S. Coast and Geodetic Survey Special Publication no. 232, 82 p. Vander Meulen, D.B., Rytuba, J.J., King, H.D., Plouff, D., and Scott, D.F., 1987, Mineral resources of the Honeycombs Wilderness Study area, Malheur County, Oregon: U.S. Geological Survey bulletin 1741-A, $15 \mathrm{p}$.

Woollard, G.P., and Rose, J.C., 1963, International gravity measurements: Society of Exploration Geophysicists, Tulsa, Okla., 518 p. 
Table 1.--Base station descriptions

The following list provides the latitude, longitude, observed gravity in milligals, elevation in feet, and descriptions for each base station. The number in parentheses after the base name indicates the number of stations tied to that base. Base station names that begin with the letters $\mathrm{CH}$ retain the name and description from the California Base Network (Chapman, 1966). The following abbreviations are used: BM, level line bench mark; CGS, bench mark originally established by the U.S. Coast and Geodetic Survey (now the National Geodetic Survey); and E, N, S, and $W$ are compass directions east, north, south, and west, respectively.

\section{$\mathrm{CH} 15(294) \quad 41^{\circ} 31.78^{\prime} \quad 120^{\prime} 10.05^{\prime} \quad 4629.2 \mathrm{ft} \quad 979,859.11 \mathrm{mGal}$}

$0.2 \mathrm{mi} E$ of Main Street in Cedarville on highway to Vya, Nev. About $75 \mathrm{ft} \mathrm{NE}$ of deserted house. About $30 \mathrm{ft} S$ of centerline of highway. On ground $1 \mathrm{ft} S$ of and $0.7 \mathrm{ft}$ below CGS BM X158. Inasmuch as this station is located at the periphery of the network coverage, the accuracy of this value is no better than $0.1 \mathrm{mGal}$.

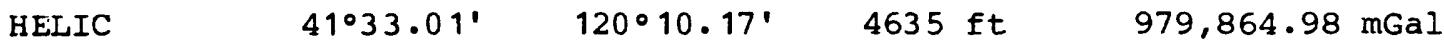

At Cedarville Airport about $1.3 \mathrm{mi} \mathrm{N}$ of town. Between hangar and Pilots' Lounge. $1 \mathrm{ft} \mathrm{W}$ of phone booth. On flat rock. Elevation interpolated from topographic map. Tied to base station $\mathrm{CH} 15$ with an accuracy of $0.02 \mathrm{mGal}$.

DENIO(223) $\quad 41^{\circ} 59.70^{\prime} \quad 118^{\circ} 34.20^{\prime} \quad 4215 \mathrm{ft} \quad 979,932.18 \mathrm{mGal}$

$E$ side of main N-S road. About $160 \mathrm{ft} S$ of Oregon State Line. $5 \mathrm{ft} S$ of SE door to Post uffice. On concrete porch. $1 \mathrm{ft}$ NW of indentation where marker for ACIC-2352-1 (Jablonski, 1974) was located. Elevation interpolated from topographic map.
P229
$41 \circ 57.10^{\prime}$
$118^{\circ} 37.49^{\prime}$
$4218 \mathrm{ft}$
$979,925.52 \mathrm{mGal}$

At Denio Junction, $2.9 \mathrm{mi} S$ of Post office at Denio. In line with the centerline of the highway to the $\mathrm{W}$ at Tee intersection. $5 \mathrm{ft} \mathrm{W}$ of $\mathrm{W}$ edge of N-S highway. Spot elevation. Tied to base station DENIO with an accuracy of $0.02 \mathrm{mGal}$.

Gerlach, Nev.

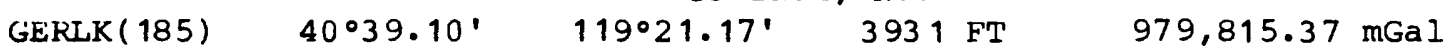
About $150 \mathrm{ft}$ SE of highway. $0.5 \mathrm{ft} \mathrm{SE}$ of $\mathrm{S}$ corner of railroad depot. $0.5 \mathrm{ft}$ $\mathrm{NE}$ of $\mathrm{SW}$ edge of concrete apron. On epoxy remnants of removed marker for ACIC-2350-1 (Jablonski, 1974). Ground vibration causing about 0.02-mGal deflection was observed during all readings.

\section{JORVL(21) $\quad 42^{\circ} 58.64^{\prime} \quad 117^{\circ} 03.14^{\prime} \quad 4390 \mathrm{ft} \quad 980,036.90 \mathrm{mGal}$}

W side of U.S. Highway 95 near NW edge of town. At NE corner of concrete stoop at $S$ entrance of Jordan Valley school. On ACIC-4608-1 (Jablonski, 1974), but marker is gone. 


\begin{abstract}
McDermitt, Nev.
$\operatorname{MCDE}(188) \quad 41^{\circ} 59.91^{\prime} \quad 117^{\circ} 43.04^{\prime} \quad 4429.1 \mathrm{ft} \quad 979,905.72 \mathrm{mGal}$

$E$ of and about $2 \mathrm{ft}$ above US Highway 95 . About $50 \mathrm{ft} S$ of road to $E$ at oregon stateline. About centerline of small house to the $E$, which has served as a Post office. E of small wall. On CGS BM Z129. Tied to base station MCDRA with an accuracy of $0.02 \mathrm{mGal}$.
\end{abstract}

MCDRA $\quad 41^{\circ} 59.91^{\prime} \quad 117 \circ 42.91^{\prime} \quad 4430 \mathrm{ft} \quad 979,905.90 \mathrm{mGal}$

$0.1 \mathrm{mi} E$ along stateline road from U.S. Highway 95. At SW corner of the E porch at NE corner of the gymnasium of the McDermitt School. S of double doors. On ACIC-2354-1 (Jablonski, 1974). On slightly shaky concrete slab. Elevation interpolated from topographic map.

Susanville, Calif.
$\mathrm{CH} 23(42)$
$40^{\circ} 24.91^{\prime}$
$120^{\circ} 38.74^{\prime}$
$4165 \mathrm{ft}$
$979,795.46 \mathrm{mGal}$

$\mathbf{N}$ side of Main Street between North Weatherlow and Grand Streets. Opposite the High School. On sidewalk $1 \mathrm{ft} S$ of $W$ end of steps in front of veterans Menorial Building.

HELIS $\quad 40^{\circ} 22.58^{\prime} \quad 120^{\circ} 34.66^{\prime} \quad 4135 \mathrm{ft} \quad 979,784.21 \mathrm{mGal}$

About $5 \mathrm{mi} S E$ of Susanville. $1 \mathrm{ft} \mathrm{SW}$ of $\mathrm{NW}$ corner of main airport building. Left end of 8-in wall in front of building. Elevation interpolated from topographic map. Tied to base station $\mathrm{CH} 23$ with an accuracy of $0.02 \mathrm{mGal}$.

WINNA(67) $\quad 40^{\circ} 54.22^{\prime} \quad 117^{\circ} 48.22^{\prime} \quad 4298 \mathrm{ft} \quad 979,810.48 \mathrm{mGal}$

About $4 \mathrm{mi} W$ of Winnemucca. $3 \mathrm{ft} W$ of door against wall of the Air Service building at the Winnemucca wunicipal Airport. $3 \mathrm{ft} \mathrm{E}$ of phone booth. Station ACIC 0474-1 (Jablonski, 1974).

$$
\text { P164 40.58.63' 117044.74' } 4344^{\prime} \mathrm{ft} \quad 979,829.20 \mathrm{mGal}
$$

Take $\mathrm{N}$ fork of first road to the $\mathrm{N}$ of Humboldt River for $0.25 \mathrm{mi}$ from U.S. Highway 95 . Gravel road junction $0.1 \mathrm{mi} \mathrm{S}$ of Welch Drive and Maple Circle intersection. $30 \mathrm{ft}$ in SE corner of intersection. $2.5 \mathrm{ft} \mathrm{N}$ of $1 / 8-$ in by $3-$ in by 4-ft post marking underground phone cable. Spot elevation $(1323 \mathrm{~m})$. Tied to base station WINNA with an accuracy of $0.03 \mathrm{mGal}$. 
Table 2.--Location description code (digit one)

[The number indicates the total number of gravity stations for which the code was used.]

\begin{tabular}{|c|c|c|}
\hline Code & Number & sxplanation \\
\hline B & 92 & $\begin{array}{l}\text { Un level-line bench mark or other permanent mark incorporated into } \\
\text { U.S. Geological Survey vertical control system, including National } \\
\text { Geodetic Survey bench marks. }\end{array}$ \\
\hline in & 193 & Near level-line bench mark described in the B-category \\
\hline $\mathrm{v}$ & 9 & $\begin{array}{l}\text { On vertical angle bench mark in U.S. Geological survey control } \\
\text { system. }\end{array}$ \\
\hline $\mathrm{H}$ & 3 & Near vertical angle bench mark. \\
\hline $\mathrm{D}$ & 15 & $\begin{array}{l}\text { Near assumed location of any of the above marks that was destroyed } \\
\text { or not found. }\end{array}$ \\
\hline $\mathbf{P}$ & 89 & Near surveyed elevation with or without a permanent mark. \\
\hline F & 1 & $\begin{array}{l}\text { Near a location with or without a mark, at which a surveyed } \\
\text { elevation is shown on a published topographic map. }\end{array}$ \\
\hline G & 585 & $\begin{array}{l}\text { Near a location (on a manuscript or published map) at which a spot } \\
\text { elevation is determined by photogrammetry; near a doubtful F- } \\
\text { location. }\end{array}$ \\
\hline W & 5 & $\begin{array}{l}\text { Edge of lake, canal, or reservoir; interpolated elevation or } \\
\text { elevation provided for water of dam at unknown height relative } \\
\text { to present level. }\end{array}$ \\
\hline Q & 16 & $\begin{array}{l}\text { Elevation determined by interpolation of topographic contours that } \\
\text { intersect intermittent drainage, stream, or river. }\end{array}$ \\
\hline c & 12 & $\begin{array}{l}\text { Elevation determined by topographic contour interpolation at less } \\
\text { precise location than defined by " } 2 \text { ". }\end{array}$ \\
\hline
\end{tabular}


Table 3.--Accuracy of elevation (digit two)

[The number after the numerical code indicates the total number of stations for which the code was used in tables of this report. The error of the Bouguer gravity anomaly is $0.06 \mathrm{mGal} / \mathrm{ft}$. The uncertainty of horizontal location tends to decrease the elevation accuracy.]

\begin{tabular}{|c|c|c|c|c|}
\hline Code & Number & $\begin{array}{l}\text { Accuracy } \\
\quad(f t)\end{array}$ & $\begin{array}{l}\text { Bouguer } \\
\text { anomaly } \\
(m G a l)\end{array}$ & Examples \\
\hline 1 & 194 & 0.2 & 0.01 & $\begin{array}{l}\text { On or tied to level-line bench mark by } \\
\text { surveying. }\end{array}$ \\
\hline 2 & 78 & 0.5 & 0.03 & $\begin{array}{l}\text { Elevation difference hand-leveled to nearby } \\
\text { bench mark. }\end{array}$ \\
\hline 3 & 119 & 1.0 & 0.06 & Near bench mark. \\
\hline 4 & 12 & 2.0 & 0.12 & $\begin{array}{l}\text { On or near vertical angle bench mark; flat area } \\
\text { near level-line bench mark that was not found. }\end{array}$ \\
\hline 5 & 39 & 5.0 & 0.3 & $\begin{array}{l}\text { Near surveyed spot elevation on topographic map; } \\
\text { elevation from map with } 10-\mathrm{ft} \text { contour interval. }\end{array}$ \\
\hline 6 & 460 & $10 \cdot 0$ & 0.6 & $\begin{array}{l}\text { Photogrammetric elevation or contour } \\
\text { interpolation on map with } 20-\mathrm{ft} \text { contour } \\
\text { interval. }\end{array}$ \\
\hline 7 & 115 & 20.0 & 1.2 & $\begin{array}{l}\text { Uncertain location of photogrammetric spot } \\
\text { elevation. }\end{array}$ \\
\hline 8 & 3 & 50.0 & 3.0 & $\begin{array}{l}\text { Contour interpolation along road or stream on } \\
\text { map with } 80-f t \text { contour interval; doubtful } \\
\text { combination of elevation and horizontal } \\
\text { location. }\end{array}$ \\
\hline
\end{tabular}


Table 4.--Accuracy of horizontal location (digit 3)

[The number after the numerical code indicates the total number of stations for which the code was used in tables of this report. The error of the Bouguer gravity anomaly is based on the assumption that all of the location error is along a north-south component of direction.]

\begin{tabular}{|c|c|c|c|c|}
\hline Code & Number & $\begin{array}{l}\text { Accuracy } \\
\quad(f t)\end{array}$ & $\begin{array}{l}\text { Bouguer } \\
\text { anomaly } \\
\text { (mGal) }\end{array}$ & Examples \\
\hline 1 & 10 & 42 & 0.01 & Near vertical angle bench mark. \\
\hline 2 & 290 & 84 & 0.02 & $\begin{array}{l}\text { Near permanent mark on map such as bench mark, } \\
\text { section corner, or well. }\end{array}$ \\
\hline 3 & 361 & 210 & 0.05 & Road intersection or stream fork. \\
\hline 4 & 296 & 420 & 0.1 & Broad road curve or gentle hillcrest. \\
\hline 5 & 62 & 840 & 0.2 & $\begin{array}{l}\text { Location depends on odometer measurement over an } \\
\text { interval greater than } 1 \mathrm{mi} \text { or other estimate. }\end{array}$ \\
\hline 7 & 1 & 4,200 & 1.0 & Uncertain location. \\
\hline
\end{tabular}

Table 5.--Hccuracy of observed gravity (digit four)

[The number after the numerical code indicates the total number of stations for which the code was used in tables of this report. Accuracies are relative to the base station, to which the station was tied.]

\begin{tabular}{cc}
\hline Code Number Accuracy \\
$(\mathrm{mGal})$
\end{tabular}

\begin{tabular}{|c|c|c|c|}
\hline 1 & 0 & 0.01 & Base station relative to itself. \\
\hline 2 & 6 & 0.02 & Station established with multiple ties of high precision. \\
\hline 3 & 65 & 0.05 & Repeated readings with LaCoste and komberg gravity meter. \\
\hline 4 & 949 & 0.1 & $\begin{array}{l}\text { Non-repeated reading with LaCoste and Romberg } \\
\text { gravity meter }\end{array}$ \\
\hline
\end{tabular}


Tabie 6--Gravity data collected in Mevada.

\begin{tabular}{|c|c|c|c|c|c|c|c|c|c|c|c|c|c|c|c|}
\hline $\begin{array}{l}\text { TATIO } \\
\text { MANE }\end{array}$ & & & $\begin{array}{l}\text { ITUDE } \\
\text { MIN }\end{array}$ & $\begin{array}{l}\text { LONG } \\
\text { DEC }\end{array}$ & $\begin{array}{l}\text { GITUDE } \\
\text { MIN }\end{array}$ & $\begin{array}{l}\text { ELEV } \\
\text { FEET }\end{array}$ & $\begin{array}{l}\text { OBS GRAV } \\
\text { MGAL }\end{array}$ & $\begin{array}{l}\text { FREE } \\
\text { AIR }\end{array}$ & $\begin{array}{l}\text { TERP } \\
\text { HWND }\end{array}$ & $\begin{array}{l}\text { IN } \\
\text { ORP }\end{array}$ & $\begin{array}{l}\text { BOUG } \\
\text { ANOM }\end{array}$ & $\begin{array}{l}\text { ISOS } \\
\text { ANOM }\end{array}$ & $\begin{array}{l}\text { ACC } \\
\text { CODE }\end{array}$ & $\begin{array}{r}\text { DATE } \\
M-D-Y\end{array}$ & $\begin{array}{l}\text { BASE } \\
\text { WAME }\end{array}$ \\
\hline 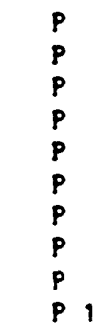 & $\begin{array}{c}1 \\
2 \\
3 \\
4 \\
5 \\
6 \\
7 \\
8 \\
9 \\
10\end{array}$ & $\begin{array}{l}40 \\
40 \\
40 \\
40 \\
40 \\
40 \\
40 \\
40 \\
40 \\
40\end{array}$ & $\begin{array}{l}16.02 \\
16.77 \\
17.59 \\
18.39 \\
19.32 \\
20.14 \\
20.86 \\
26.02 \\
25.87 \\
25.66\end{array}$ & $\begin{array}{l}119 \\
119 \\
119 \\
119 \\
119 \\
119 \\
119 \\
119 \\
119 \\
119\end{array}$ & $\begin{array}{l}21.89 \\
21.69 \\
21.39 \\
21.05 \\
20.68 \\
20.40 \\
20.29 \\
19.96 \\
17.98 \\
17.29\end{array}$ & $\begin{array}{l}3810.0 \\
3837.6 \\
3871.5 \\
3897.6 \\
4062.7 \\
4171.3 \\
4230.1 \\
4638.1 \\
5055.0 \\
5295.0\end{array}$ & $\begin{array}{l}979800.56 \\
979802.45 \\
979809.21 \\
979809.97 \\
979800.16 \\
979794.14 \\
979792.92 \\
979775.84 \\
979753.38 \\
979742.96\end{array}$ & $\begin{array}{r}-33.9 \\
-30.6 \\
-21.9 \\
-19.8 \\
-15.5 \\
-12.5 \\
-9.3 \\
4.3 \\
21.3 \\
33.7\end{array}$ & $\begin{array}{l}0.08 \\
0.19 \\
0.18 \\
0.19 \\
0.48 \\
0.08 \\
0.08 \\
0.08 \\
0.39 \\
0.39\end{array}$ & $\begin{array}{l}2.0 \\
1.8 \\
1.4 \\
1.3 \\
1.4 \\
0.9 \\
0.9 \\
1.1 \\
2.1 \\
3.2\end{array}$ & $\begin{array}{l}163.1 \\
160.9 \\
153.7 \\
152.7 \\
154.0 \\
155.2 \\
153.9 \\
154.2 \\
150.1 \\
145.2\end{array}$ & $\begin{array}{l}-11.9 \\
-9.8 \\
-2.4 \\
-1.4 \\
-2.7 \\
-3.9 \\
-2.6 \\
-2.7 \\
1.3 \\
6.2\end{array}$ & $\begin{array}{l}D 334 \\
B 124 \\
\text { N224 } \\
B 124 \\
B 124 \\
B 124 \\
B 123 \\
B 122 \\
\text { C634 } \\
\text { C634 }\end{array}$ & $\begin{array}{l}61884 \\
61884 \\
61884 \\
61884 \\
61884 \\
61884 \\
62084 \\
62084 \\
61984 \\
61984\end{array}$ & $\begin{array}{l}\text { GERLK } \\
\text { GERLK } \\
\text { GERLK } \\
\text { CERLK } \\
\text { GERLK } \\
\text { GERLK } \\
\text { GERLK } \\
\text { CERLK } \\
\text { GERLK } \\
\text { GERLK }\end{array}$ \\
\hline $\begin{array}{ll}P & 1 \\
P & 1 \\
P & 1 \\
P & 1 \\
P & 1 \\
P & 1 \\
P & 1 \\
P & 1 \\
P & 1 \\
P & 2\end{array}$ & $\begin{array}{l}11 \\
12 \\
13 \\
14 \\
15 \\
16 \\
17 \\
18 \\
19 \\
20\end{array}$ & $\begin{array}{l}40 \\
40 \\
40 \\
40 \\
40 \\
40 \\
40 \\
40 \\
40 \\
40\end{array}$ & $\begin{array}{l}25.75 \\
27.04 \\
26.06 \\
25.03 \\
23.87 \\
24.15 \\
23.05 \\
21.67 \\
20.01 \\
18.71\end{array}$ & $\begin{array}{l}119 \\
119 \\
119 \\
119 \\
119 \\
119 \\
119 \\
119 \\
119 \\
119\end{array}$ & $\begin{array}{l}16.28 \\
14.98 \\
13.56 \\
12.91 \\
11.28 \\
13.59 \\
14.28 \\
12.93 \\
11.42 \\
9.28\end{array}$ & $\begin{array}{l}4.0 \\
2.1 \\
9.5 \\
3.4 \\
6.0 \\
4.4 \\
1.0 \\
4.6 \\
3.5 \\
9.4\end{array}$ & $\begin{array}{l}01.76 \\
20.55 \\
49.07 \\
59.86 \\
69.68 \\
50.73 \\
51.47 \\
64.07 \\
73.99 \\
56.08\end{array}$ & $\begin{array}{r}54.3 \\
47.5 \\
34.0 \\
23.2 \\
0.2 \\
29.6 \\
26.1 \\
6.2 \\
5.3 \\
-12.0\end{array}$ & $\begin{array}{l}1.69 \\
0.28 \\
0.48 \\
1.08 \\
0.08 \\
0.38 \\
0.18 \\
0.09 \\
0.09 \\
0.08\end{array}$ & $\begin{array}{l}5.2 \\
2.2 \\
2.3 \\
3.0 \\
0.5 \\
2.8 \\
2.5 \\
0.6 \\
0.3 \\
0.3\end{array}$ & & $\begin{array}{r}6.2 \\
5.3 \\
7.8 \\
6.1 \\
-6.7 \\
7.1 \\
5.3 \\
-3.8 \\
-0.3 \\
-17.9\end{array}$ & & $\begin{array}{l}1984 \\
1984 \\
1984 \\
1984 \\
1984 \\
1984 \\
1984 \\
1984 \\
1984 \\
1984\end{array}$ & $\begin{array}{l}\text { GERLK } \\
\text { GERLK } \\
\text { GERLK } \\
\text { GERLK } \\
\text { GERLK } \\
\text { GERLK } \\
\text { GERLK } \\
\text { GERLK } \\
\text { GERLK } \\
\text { GERLK }\end{array}$ \\
\hline $\begin{array}{ll}P & 2 \\
P & 2 \\
P & 2 \\
P & 2 \\
P & 2 \\
P & 2 \\
P & 2 \\
P & 2 \\
P & 2 \\
P & 3\end{array}$ & $\begin{array}{l}21 \\
22 \\
23 \\
24 \\
25 \\
26 \\
27 \\
28 \\
29 \\
30\end{array}$ & $\begin{array}{l}40 \\
40 \\
40 \\
40 \\
40 \\
40 \\
40 \\
40 \\
40 \\
40\end{array}$ & $\begin{array}{l}18.50 \\
18.33 \\
18.03 \\
17.91 \\
19.75 \\
21.22 \\
21.69 \\
19.97 \\
18.64 \\
17.21\end{array}$ & $\begin{array}{l}11 \\
11 \\
11 \\
11 \\
11 \\
11 \\
11 \\
11 \\
11 \\
11\end{array}$ & $\begin{array}{l}10.36 \\
11.53 \\
13.02 \\
13.72 \\
14.08 \\
14.36 \\
15.39 \\
15.90 \\
14.66 \\
14.75\end{array}$ & $\begin{array}{l}4565.1 \\
4595.0 \\
4586.3 \\
4545.8 \\
4747.4 \\
4934.4 \\
5270.0 \\
5292.0 \\
4681.8 \\
4527.6\end{array}$ & & $\begin{array}{r}-7.2 \\
-2.0 \\
-3.5 \\
-7.8 \\
2.9 \\
15.1 \\
32.0 \\
30.1 \\
0.2 \\
-8.7\end{array}$ & $\begin{array}{l}0.0 r \\
0.0 r \\
0.0 r \\
0.0 r \\
0.0 r \\
0.3 r \\
0.28 \\
0.89 \\
0.08 \\
0.18\end{array}$ & $\begin{array}{l}0.3 \\
0.3 \\
0.3 \\
0.3 \\
0.6 \\
1.3 \\
2.5 \\
3.4 \\
0.5 \\
0.5\end{array}$ & $\begin{array}{l}-16 \\
-15 \\
-16 \\
-16 \\
-15 \\
-15 \\
-1 \\
-12 \\
-16 \\
-16\end{array}$ & $\begin{array}{r}-12.5 \\
-8.4 \\
-9.5 \\
-12.4 \\
-8.5 \\
-1.9 \\
4.6 \\
2.7 \\
-9.0 \\
-12.6\end{array}$ & & & $\begin{array}{l}\text { GERIK } \\
\text { GERLK } \\
\text { GERLK } \\
\text { GERLK } \\
\text { GER:K } \\
\text { GERLK } \\
\text { GERLK } \\
\text { GERLK } \\
\text { GERLK } \\
\text { GERLK }\end{array}$ \\
\hline 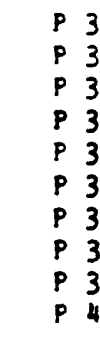 & $\begin{array}{l}31 \\
32 \\
33\end{array}$ & $\begin{array}{l}40 \\
40 \\
40 \\
40 \\
40 \\
40 \\
40 \\
40 \\
40 \\
40\end{array}$ & $\begin{array}{l}16.18 \\
14.82 \\
13.03 \\
15.63 \\
16.82 \\
17.85 \\
18.50 \\
18.65 \\
19.38 \\
21.10\end{array}$ & $\begin{array}{l}119 \\
119 \\
119 \\
119 \\
119 \\
119 \\
119 \\
119 \\
119 \\
119\end{array}$ & $\begin{array}{l}15.03 \\
15.19 \\
16.00 \\
16.00 \\
16.83 \\
17.78 \\
18.71 \\
20.21 \\
18.14 \\
18.57\end{array}$ & $\begin{array}{l}4459.0 \\
4377.0 \\
4256.0 \\
4183.0 \\
3972.0 \\
3917.0 \\
3948.0 \\
3927.0 \\
4107.0 \\
4425.0\end{array}$ & & $\begin{array}{l}-5.6 \\
-6.6 \\
-8.1 \\
-9.3 \\
-14.6 \\
-19.4 \\
-23.4 \\
-22.5 \\
-12.2 \\
-1.5\end{array}$ & $\begin{array}{l}0.19 \\
0.49 \\
1.08 \\
0.19 \\
0.29 \\
0.19 \\
0.09 \\
0.09 \\
0.49 \\
0.39\end{array}$ & $\begin{array}{l}0.8 \\
1.5 \\
3.3 \\
1.2 \\
1.5 \\
1.3 \\
1.1 \\
1.0 \\
2.6 \\
2.8\end{array}$ & $\begin{array}{l}-15 \\
-15 \\
-15 \\
-15 \\
-14 \\
-15 \\
-15 \\
-15 \\
-15 \\
-15\end{array}$ & $\begin{array}{r}-7.0 \\
-4.3 \\
0.1 \\
-0.7 \\
1.6 \\
-1.5 \\
-6.9 \\
-5.4 \\
0.4 \\
0.4\end{array}$ & $\begin{array}{l}C 634 \\
0634 \\
C 624 \\
C 624 \\
C 634 \\
C 644 \\
0634 \\
C 624 \\
c 754 \\
0754\end{array}$ & $\begin{array}{l}1984 \\
1984 \\
1984 \\
1984 \\
1984 \\
1984 \\
1984 \\
1984 \\
2084 \\
2084\end{array}$ & $\begin{array}{l}\text { GERIK } \\
\text { GERLK } \\
\text { GERLK } \\
\text { GERLK } \\
\text { GERLK } \\
\text { GERLK } \\
\text { GERLK } \\
\text { GERLK } \\
\text { GERLK } \\
\text { GERLK }\end{array}$ \\
\hline $\begin{array}{ll}P & 4 \\
P & 4 \\
P & 4 \\
P & 4 \\
P & 4 \\
P & 4 \\
P & 4 \\
P & 4 \\
P & 4 \\
P & 5\end{array}$ & $\begin{array}{l}2 \\
43 \\
44 \\
45 \\
46 \\
47\end{array}$ & $\begin{array}{l}40 \\
40 \\
40 \\
40 \\
40 \\
40 \\
40 \\
40 \\
40\end{array}$ & $\begin{array}{l}21.63 \\
22.45 \\
23.47 \\
24.22 \\
24.26 \\
25.28 \\
26.74 \\
50.21 \\
58.45 \\
5.17\end{array}$ & $\begin{array}{l}119 \\
119 \\
119 \\
119 \\
119 \\
119 \\
119 \\
119 \\
119 \\
119\end{array}$ & $\begin{array}{l}20.15 \\
20.02 \\
19.94 \\
17.93 \\
19.96 \\
19.96 \\
19.99 \\
14.31 \\
9.05 \\
7.03\end{array}$ & $\begin{array}{l}4324.5 \\
405.8 \\
4530.5 \\
5042.0 \\
4597.5 \\
4673.2 \\
4601.4 \\
4081.0 \\
3972.0 \\
3969.0\end{array}$ & $\begin{array}{l}38.52 \\
33.45 \\
7.30 \\
32.06 \\
1.25 \\
2.59 \\
31.46 \\
65.99 \\
52.41\end{array}$ & $\begin{array}{r}-6.0 \\
-4.6 \\
-0.6 \\
21.2 \\
1.5 \\
5.4 \\
5.4 \\
6.0 \\
-20.1 \\
-17.9\end{array}$ & $\begin{array}{l}0.08 \\
0.18 \\
0.18 \\
0.39 \\
0.18 \\
0.08 \\
0.18 \\
0.18 \\
0.09 \\
0.08\end{array}$ & $\begin{array}{l}1.0 \\
1.1 \\
1.3 \\
3.7 \\
1.4 \\
1.1 \\
1.0 \\
0.2 \\
1.0 \\
0.9\end{array}$ & $\begin{array}{l}-153.8 \\
-155.1 \\
-155.1 \\
-148.5 \\
-155.3 \\
-154.2 \\
-151.9 \\
-134.2 \\
-155.8 \\
-153.6\end{array}$ & $\begin{array}{r}-2.5 \\
-3.7 \\
-3.7 \\
2.8 \\
-3.8 \\
-2.8 \\
-0.3 \\
20.1 \\
-0.5 \\
3.7\end{array}$ & $\begin{array}{l}\text { B124 } \\
\text { B124 } \\
\text { B124 } \\
\text { O754 } \\
\text { B124 } \\
\text { B124 } \\
\text { B124 } \\
\text { O534 } \\
\text { C534 } \\
\text { O533 }\end{array}$ & $\begin{array}{l}62084 \\
62084 \\
62084 \\
62084 \\
62084 \\
62084 \\
62084 \\
62084 \\
62084 \\
62584\end{array}$ & $\begin{array}{l}\text { GERLK } \\
\text { CERLK } \\
\text { GERLK } \\
\text { GERLK } \\
\text { GERLK } \\
\text { GERLK } \\
\text { GERLK } \\
\text { GERLK } \\
\text { CERLK } \\
\text { CERLK }\end{array}$ \\
\hline
\end{tabular}


Table 6--(continued)

STATIOI LATITUDE LONGITUDE HAME DKG MIN DEG MIN

MEV OBS GRAV FEET MGAL

P $51 \quad 4114.53 \quad 119 \quad 9.83$ $P 52 \quad 1 \quad 22.94 \quad 119 \quad 10.07$

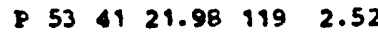
\begin{tabular}{lllll}
\hline & $44 \quad 47.52 \quad 119 \quad 16.64$
\end{tabular}

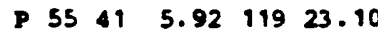
$\begin{array}{llllll}P & 56 & 41 & 8.43 & 119 & 22.20\end{array}$ \begin{tabular}{l}
$P 544 \quad 9.27 \quad 119 \quad 18.83$ \\
\hline 584
\end{tabular} $\begin{array}{llllll}P & 58 \quad 41 & 8.48 \quad 119 & 17.96\end{array}$ $\begin{array}{llllll}P & 59 & 41 & 7.80 & 119 & 17.58\end{array}$ $\begin{array}{llllll}P & 60 & 41 & 6.51 & 119 & 17.30\end{array}$

$\begin{array}{lllll}P & 61 & 5.27 & 119 & 17.58\end{array}$ $\begin{array}{llllll}P & 62 & 41 & 6.91 & 119 & 16.41\end{array}$ \begin{tabular}{llllll}
\hline & 41 & 7.79 & 119 & 16.69
\end{tabular} $\begin{array}{llllll}\text { P } 64 \quad 11 & 9.66 & 119 & 15.94\end{array}$ \begin{tabular}{llllll}
\hline & 651 & $9.60 \quad 119$ & 14.25
\end{tabular} \begin{tabular}{llllll}
\hline P & 41 & $8.10 \quad 119$ & 14.68
\end{tabular}

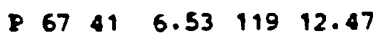
$\begin{array}{lllllll}P & 68 & 41 & 5.16 & 119 & 11.94\end{array}$ $\begin{array}{llllll}P & 69 & 41 & 5.39 & 119 & 10.57\end{array}$ $P$
$P 41 \quad 5.36 \quad 119 \quad 8.93$

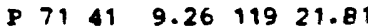
$7241 \quad 9.86 \quad 11921.38$ $\begin{array}{lllll}P & 73 & 41 & 10.20 \quad 119 & 20.50\end{array}$

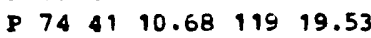
\begin{tabular}{llllll}
\hline & 75 & 41 & $11.13 \quad 119$ & 18.88
\end{tabular} $\begin{array}{llllll}76 & 41 & 12.06 \quad 119 & 19.07\end{array}$ $\begin{array}{lllll}77 & 41 & 12.87 & 119 & 18.94\end{array}$ r $7641 \quad 11.95119 \quad 17.40$ $\begin{array}{lllll}P & 79 & 41 & 13.37 \quad 119 & 18.26\end{array}$ P $8041 \quad 14.32 \quad 119 \quad 18.32$

$P 141 \quad 14.92 \quad 119 \quad 17.93$ $\begin{array}{llllll}P & 41 & 15.50 & 119 & 18.22\end{array}$

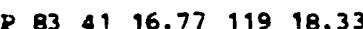
$P 4418.19 \quad 119 \quad 21.18$ $\begin{array}{llllll}P & 85 & 41 & 17.56 & 119 & 18.26\end{array}$ ץ $86 \quad 41 \quad 18.81 \quad 119 \quad 16.72$ $\begin{array}{llllll}P & 87 & 41 & 18.36 & 119 & 16.76\end{array}$

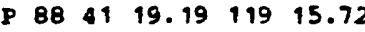
\begin{tabular}{l}
$P$ \\
$P 41$ \\
\hline
\end{tabular} P $9041 \quad 17.46 \quad 1186.08$

$P 91 \quad 41 \quad 18.88 \quad 118 \quad 56.83$ \begin{tabular}{l}
$P$ \\
\hline
\end{tabular} $119.66 \quad 118 \quad 58.62$ $P$
$P$ $121.10 \quad 119 \quad 0.85$ $\begin{array}{lllll}P & 41 & 15.55 & 119 & 1.85\end{array}$ $P$
$P \quad 1 \quad 15.65 \quad 119 \quad 5.18$ \begin{tabular}{llllll}
\hline & 96 & 41 & 17.90 & 119 & 12.23
\end{tabular} P97Q $41 \quad 17.50 \quad 119 \quad 14.20$

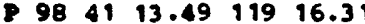
$P 9941 \quad 14.20 \quad 119 \quad 13.78$ $\begin{array}{llll}P & \$ 1 & 12.50 \quad 119 \quad 12.28\end{array}$

\begin{abstract}
4203.7979858 .58 4474.0979856 .44 $5857.0 \quad 979776.66$ 4087.4979854 .13 $5843.0 \quad 979746.50$ 5728.1979752 .99 $5659.0 \quad 979758.83$ $5664.0 \quad 979755.45$ $5829.0 \quad 979742.91$ $6121.0 \quad 979726.58$
\end{abstract} $6330.0 \quad 979720.62$ $6079.0 \quad 979732.06$ $6079.0 \quad 979734.85$ $7104.0 \quad 979670.46$ $7043.0 \quad 979674.56$ $5941.0 \quad 979752.95$ $5755.0 \quad 979766.61$ $5059.0 \quad 979808.93$ $4172.0 \quad 979864.65$

5675.4979755 .24 5654.6979756 .45 5750.2979754 .59 $5413.5 \quad 979774.57$ 5168.5979791 .16 $5070.2 \quad 979799.70$ 5022.4979804 .09 $5279.0 \quad 979786.37$ 5000.1979809 .10 $4985.0 \quad 979815.98$

4952.2979818 .90 $4941.6 \quad 979819.63$ $4912.5 \quad 979817.95$ $5921.0 \quad 979753.73$ 4902.8979819 .04 4926.0979819 .75 4912.3979818 .90 4939.0979822 .14 4443.3979857 .20 4412.6979859 .18

$4536.0 \quad 979853.23$ 4876.0979833 .68 $5585.0 \quad 979796.24$ $7707.0 \quad 979634.5$ $6257.0 \quad 979735.99$ $4951.0 \quad 979816.53$ 5050.0979811 .85 $5547.0 \quad 979772.26$ 6215.0979724 .52 $5635.0 \quad 979766.70$
$6010.0 \quad 979735.48$
FREE TERRAIN BOUG AIR HAND COAP ANOM

$\begin{array}{rlll}-26.2 & 0.0 f & 1.6 & -169.3 \\ -15.5 & 0.0 f & 1.5 & -167.9 \\ 36.2 & 0.4 f & 2.3 & -162.8 \\ -1.2 & 0.1 f & 0.5 & -141.4 \\ 28.7 & 0.3 f & 2.0 & -170.1 \\ 20.6 & 0.1 f & 1.2 & -175.0 \\ 18.7 & 0.3 f & 1.5 & -174.3 \\ 17.0 & 0.0 f & 1.5 & -176.2 \\ 21.0 & 0.1 f & 1.8 & -177.5 \\ 34.0 & 0.2 f & 2.7 & -173.5\end{array}$

$34.30 .2 f$

4.10 .8

$33.70 .2 f$

$33.6 \quad 0.65$

$65.70 .3 f$

$\begin{array}{lll}66.3 & 0.4 f\end{array}$

$43.52 .2 f$

$41.70 .7 f$

$18.21 .7 f$

$-9.4 \quad 0.15$

$16.7 \quad 0.2 f$

$15.10 .2 f$

$21.70 .2 f$

$9.31 .5 f$

$2.2 \cdot 1.2 f$

0.10 .16

$\begin{array}{ll}-1.2 & 0.1 f\end{array}$

6.60 .11

$0.90 .1 f$

$2.7-169.4$

$4.3-166.0$

$2.7-172.5$

$3.2-172.0$

$6.2-171.9$

$5.4-170.0$

$6.1-154.6$

$4.4-151.7$

$4.3-151.5$

$1.8-151.1$

$1.1-177.3$

$1.1-178.1$

$1.1-174.8$

$2.8-174.0$

$2.8-172.7$

$1.5-172.6$

$1.4-172.6$

$1.3-173.6$

$1.2-169.8$

$5.0 \quad 0.1 f \quad 1.1-165.3$

$3.9 \quad 0.1 f \quad 1.2-165.1$

$2.8 \quad 0.4 f \quad 1.4-165.7$

$\begin{array}{llll}-3.5 & 0.0 f & 0.9 & -171.5\end{array}$

$24.9 \quad 0.1 f \quad 1.3-177.3$

$\begin{array}{llll}-4.5 & 0.4 f & 1.3 & -171.9\end{array}$

$\begin{array}{llll}-3.5 & 0.9 f \quad 2.1 & -170.8\end{array}$

$\begin{array}{lllll}-5.0 & 0.1 f & 0.8 & -173.1\end{array}$

$\begin{array}{llll}-0.4 & 0.5 f & 1.3 & -169.0\end{array}$

$\begin{array}{lll}-10.4 & 0.11 \quad 1.5-161.8\end{array}$

$\begin{array}{llll}-10.3 & 0.9 f & 2.5 & -159.6\end{array}$

$\begin{array}{llll}-6.8 & 1.9 f \quad 4.4 & -158.4\end{array}$

$\begin{array}{lllll}4.5 & 1.36 & -8 & -158.4\end{array}$

$31.5 \quad 3.3 f \quad 6.3-154.1$

$77.53 .8 f \quad 19.4-167.5$

$42.6 \quad 6.7 f \quad 14.5-157.9$

$\begin{array}{llll}-3.0 & 1.8 f \quad 3.0 & -170.3\end{array}$

$2.20 .3 f \quad 1.0-170.4$

$\begin{array}{lllll}15.3 & 0.2 f & 1.6 & -173.7\end{array}$

$29.3 \quad 3.8 f \quad 0.7-175.5$

$19.51 .4 f \quad 4.4-169.8$
IsOS ACC NOA CODE M-D-Y MAME

\section{$-7.18123$}

$-2.16523$

0.26533

12.9 N1 23

$-7.86534$

-12.0 N122

$-11.96634$

$-14.46624$

$-16.2 G 634$

-12.9 C634

$-9.2 \quad 0634$

$-5.56634$

$-11.56634$

-10.5 G634

$-11.36644$

$-9.96634$

$4.6 \mathrm{E644}$

6.76644

6.86644

6.80734

$-14.0 N 124$

$-14.8 N 124$

-11.7 N 124

-10.8 N224

-9.4 D444

$-9.1 N 124$

$-8.6 \times 123$

$-10.5 c 634$

$-5.8 \times 124$

-1.0 N224

$-0.7 \times 224$

$-1.0 \times 124$

$-6.3 \quad B 123$

-11.2 G644

$-6.4 \times 124$

$-4.8 \times 123$

-7.7 B 124

$-3.6 \times 544$

$-2.2 N 123$

$-0.2 \times 124$

1.96534

2.56654

8.0 C644

$-8.3 \quad 6634$

$2.8 \quad 6634$

$-6.20634$

$-6.0 \mathrm{cB7} 4$

$-10.5 \cos 34$

-12.9 es44

$-8.1 \mathrm{c} 64$
62084 GERLK

81786 DENIO

62584 GERLK

62684 GERLK

62184 GERLX

62684 GIRIX

62184 GERLK

62184 GEFLX

62184 GERLX

62184 GERIX

62184 GERIK

62184 GERIK

62184 GERIK

62184 GERLK

62184 GERLK

62184 GLRLK

62184 GLRLK

62184 GERIK

62184 GLELK

62184 GLRLK

62284 GIRLK

62284 GERLK 62284 GERIX

62284 GEKLK 62284 GLRLX

62284 GEKLK 62284 GLRLK 62284 GIFiK 62284 GERLK 62284 GERIL

62284 GERLK 62284 GLRLK 81185 GERLK 62284 GERLX 62284 GERIK 62284 GERLK 62284 GERLK 62284 GERLK 62584 GERLK 62384 GERLK

62384 GERIK 62384 GERIK 62384 GERLK 62484 GERLK 62484 GERLK 62484 GERLK 62484 GEFLK 62484 GERLK 62484 GERIKK 62484 GERLK 
Table 6--(continued)

\begin{tabular}{|c|c|c|c|c|c|c|c|c|c|c|c|c|c|c|}
\hline \multirow{2}{*}{$\begin{array}{l}\text { STATION } \\
\text { WWML }\end{array}$} & \multicolumn{2}{|c|}{ LATITUDE } & \multicolumn{2}{|c|}{ LONGITUDE } & \multirow{2}{*}{$\begin{array}{l}\text { DEVV } \\
\text { FEET }\end{array}$} & \multirow{2}{*}{$\begin{array}{l}\text { OBS GRAV } \\
\text { MGAL }\end{array}$} & \multirow{2}{*}{$\begin{array}{l}\text { FREE } \\
\text { AIK }\end{array}$} & \multicolumn{2}{|c|}{ TERRAIN } & \multirow{2}{*}{$\begin{array}{l}\text { DOVG } \\
\text { ANOS }\end{array}$} & \multirow{2}{*}{$\begin{array}{l}\text { IsOS } \\
\text { Mar }\end{array}$} & \multirow{2}{*}{$\begin{array}{l}\mathrm{ACC} \\
\text { CODL }\end{array}$} & \multirow{2}{*}{$\begin{array}{r}\text { DATE } \\
M-D-Y\end{array}$} & \multirow{2}{*}{$\begin{array}{l}\text { BASE } \\
\text { MAME }\end{array}$} \\
\hline & & MIN & DEG & MIN & & & & MAND & coup & & & & & \\
\hline 8101 & 41 & 11.49 & 119 & 14.14 & 1094.0 & 979669.64 & 61.1 & $.5 f$ & 9.0 & -173.4 & -12.0 & 6634 & 62484 & GERIY. \\
\hline$P 102$ & 41 & 9.62 & 119 & 11.98 & 6030.0 & 979732.82 & 27.1 & $2.3 f$ & 6.5 & -173.6 & -13.4 & 6634 & 62484 & GERLK \\
\hline$P 103$ & 41 & 7.93 & 119 & 11.11 & 5705.0 & 979764.67 & 30.9 & $3.2 f$ & 7.3 & -157.9 & 1.4 & 6534 & 62484 & GLRLK \\
\hline P104 & 41 & 10.85 & 119 & 10.12 & 5085.0 & 979798.06 & 1.7 & $0.7 \mathrm{f}$ & 3.5 & -169.7 & -9.4 & 6644 & 62484 & GERLX \\
\hline P 105 & 41 & 10.97 & 118 & 59.01 & 5568.0 & 979786.69 & 35.5 & $3.9 f$ & 8.6 & -147.2 & 9.7 & 6634 & 62484 & GERLK \\
\hline$P 106$ & 41 & 13.51 & 118 & 58.92 & 4834.0 & 979832.42 & 8.4 & $0.4 f$ & 1.8 & -156.0 & 2.2 & 6644 & 62484 & GERLK \\
\hline 2107 & 41 & 18.64 & 119 & 7.22 & 5962.0 & 979775. & 50.1 & 2.85 & 7.3 & -147.5 & 15.1 & 6634 & 62484 & GERLK \\
\hline P 10B & 41 & 16.52 & 118 & 55.89 & 4436.7 & 9798 & -5.6 & 0.71 & 2.0 & -156.3 & 2.6 & H124 & 2484 & GERIK \\
\hline P109 & 41 & 15.77 & 118 & 56. & 4488.6 & 979859 & -0.8 & $0.2 f$ & 1.5 & $-153 \cdot 8$ & 4.9 & $N 124$ & 2484 & GLRLK \\
\hline P110 & 41 & 14.92 & 118 & 56. & 4289.9 & & & 0.06 & 1.2 & & -0.5 & $N 124$ & 2484 & GERIK \\
\hline ₹111 & 41 & 14.06 & 198 & 56.19 & 4.9 & & -8.1 & $0.1 f$ & 1.0 & 5.3 & 2.6 & 124 & 484 & ERLK \\
\hline P112 & 41 & 13.26 & 118 & 56.66 & 4320.0 & 979868.27 & $-3 \cdot 6$ & $0.1 f$ & 0.8 & -151.5 & 6.2 & N124 & 2484 & GEFLK \\
\hline P113 & 41 & 12.16 & 118 & 56.66 & 4233.1 & 979876.12 & $-2 \cdot 3$ & 0.11 & 0.6 & -147.4 & 9.8 & $N 124$ & 2484 & GERIK \\
\hline P114 & 41 & 10.38 & 118 & 56.16 & 269.8 & 97987 & 6.3 & $0.1 f$ & 0.4 & -140.2 & 16.1 & N124 & 2484 & GLRLK \\
\hline 8115 & 41 & 8.86 & 118 & 55.87 & 4128.5 & & 1.2 & $0.1 f$ & 0.5 & -140.4 & 15.2 & B 123 & 2484 & GERLK \\
\hline P116 & 41 & 7.57 & 118 & 55.34 & 4001.4 & & 0.9 & $0.3 f$ & 0.8 & -136.0 & 19.1 & N124 & 2484 & GERLK \\
\hline P117 & 41 & 6.83 & 118 & 54.78 & 3995.9 & & -1.9 & 0.08 & 0.4 & 9.0 & 15.7 & N124 & 2484 & GERLK \\
\hline P118 & 41 & 5.38 & 118 & 53.63 & 3948.2 & 979 & -6.7 & $0.0 f$ & 0.3 & -1 & 11.8 & N124 & 2484 & GERL $Y$. \\
\hline P119 & 41 & 3.87 & 118 & 54.21 & 3990.8 & 15 & $-5 \cdot 7$ & $0.0 E$ & 0.2 & 2.8 & 11.0 & B124 & 2484 & GERLK \\
\hline P120 & 41 & 2.97 & 118 & 54.19 & 8.2 & 975 & -4.7 & 0.01 & 0.2 & 0.7 & 12.9 & B124 & 2484 & GERLK \\
\hline P121 & 41 & 21.27 & 119 & & & & 56.2 & $0.8 f$ & 3.9 & -163.6 & -0.7 & 6634 & & GERiK \\
\hline P122 & 41 & 19.53 & 119 & 4.41 & 6176.0 & 9797 & 57.5 & $0.0 f$ & 2.4 & -152.3 & 9.9 & 6634 & 2584 & GERLK \\
\hline 5123 & 41 & 18.00 & 119 & 4.01 & 6614.0 & 975 & 70.8 & $0.4 f$ & 4.6 & -151.6 & 9.6 & 6634 & 2584 & GERLK \\
\hline P124 & 41 & 18.42 & 119 & 2.41 & 6343.0 & & 60.8 & $0.2 f$ & 3.2 & -153.8 & 7.3 & $W 644$ & 684 & GERLK \\
\hline P125 & 41 & 18.78 & 119 & 4.56 & 6261.0 & & 60.6 & $0.1 f$ & 2.8 & 1.7 & 10.2 & W644 & 584 & EREK \\
\hline 8126 & 41 & 24.82 & 119 & 4.15 & 5234.0 & & 3.2 & $.6 f$ & 4.1 & 2.6 & -7.8 & C743 & 86 & DENIO \\
\hline$P 127$ & 41 & 24.07 & 119 & 6.98 & 3.0 & & 1.8 & $.6 f$ & 2.6 & 6.6 & -1.3 & 6634 & 84 & ERLK \\
\hline$P 128$ & 41 & 26.53 & 119 & & 1.0 & 979768 & .3 & $0.5 f$ & 2.8 & .6 & -13.0 & G534 & 84 & GERiג \\
\hline P129 & 41 & 27.22 & 119 & 10.20 & 6102.0 & 979748.44 & 3.1 & $0.2 f$ & 2.6 & -183.9 & -17.0 & 6634 & 2584 & GLRLK \\
\hline P130 & 41 & & 119 & & 5638.0 & 4.14 & & $0.2 f$ & 1.2 & 9.0 & -21.2 & 6634 & 584 & \\
\hline 139 & 41 & 28.12 & 119 & .43 & 4.0 & & 4.5 & $0.5 t$ & 1.4 & -15 & -25.5 & 6634 & 84 & $R L K$ \\
\hline P132 & 41 & 9.47 & 119 & 7.95 & 4035.5 & 979862.87 & -30.1 & 0.08 & 1.4 & -167.6 & -8.2 & 5124 & 2584 & FEFLK \\
\hline P133 & 41 & 3.71 & 119 & 7.44 & 3973.0 & 979873.64 & -16.6 & $0.0 f$ & 1.0 & -152.4 & 4.5 & G534 & 2584 & GERLK \\
\hline$P 134$ & 41 & 9.67 & 119 & 23.29 & 5844.2 & 979747.48 & 24.2 & 0.15 & 1.1 & -175.5 & -11.8 & B124 & 2684 & EFLK \\
\hline P135 & 41 & 10.16 & 119 & $24 \cdot 50$ & 6018.2 & 979737.56 & 29.9 & 0.15 & 1.1 & -175.7 & -11.7 & B124 & 62684 & GERLK \\
\hline$\nvdash 136$ & 41 & 11.29 & 119 & & 5607.0 & .89 & 16.9 & $0.4 f$ & 1.3 & -174.5 & -9.6 & N324 & 2684 & GERLK \\
\hline$P 137$ & 41 & 11.98 & 119 & 26.32 & 5596.6 & .62 & 14.6 & $0.1 f$ & 0.8 & -177.0 & -11.7 & N124 & 2684 & GERLK \\
\hline P138 & 41 & 12.51 & 119 & 27 & 5429.1 & 1.29 & 7.7 & $0.1 E$ & 0.7 & -178.3 & -12.5 & B 124 & 2684 & GERLK \\
\hline P 139 & 41 & 13.15 & 119 & 28.79 & 5301.6 & .70 & -2.8 & $0.0 f$ & 0.6 & 4.5 & -18.4 & N123 & 885 & GERLK \\
\hline & 41 & & 119 & & & 979768.80 & 0.8 & $0.1 E$ & 0.6 & & & B 124 & 62684 & GERIX \\
\hline & 41 & 9.12 & 119 & & 9.4 & & -0.3 & $1 f$ & & & & & & ERLK \\
\hline 142 & 41 & 7.59 & 119 & & 06.2 & & & 0.08 & 0.7 & & -20.7 & 124 & 84 & ERLK \\
\hline 143 & 41 & 6.91 & 119 & 32.32 & 546.4 & & 1.9 & $0.1 f$ & 0.8 & 7.9 & -23.1 & $N 124$ & 384 & GERLK \\
\hline 144 & 41 & 5.99 & 119 & 40.53 & 6084.8 & 979711.49 & 3 & $0.1 f$ & 0.9 & -191.8 & -26.5 & N123 & 85 & GERIX \\
\hline$P 149$ & 41 & 13.11 & 119 & 39.89 & 6476.0 & 979705.82 & 36.8 & $0.2 f$ & 1.9 & -183.8 & -16.9 & 6634 & 62684 & GERLK \\
\hline$P 150$ & 41 & 14.36 & 119 & 39.84 & 5863.0 & 979746.10 & 19.6 & $0.1 f$ & 0.9 & -181.0 & -13.6 & 6634 & 62684 & GLRLK \\
\hline P 151 & 41 & 14.51 & 119 & 03.11 & 5821.0 & 9797 & 19.7 & $0.0 f$ & 0.6 & -179.8 & -12.2 & 6634 & 62684 & GERLK \\
\hline P152 & 41 & 13.70 & 119 & 44.81 & 5901.0 & 979741.94 & 18.0 & $0.1 f$ & 0.7 & -184.1 & -16.7 & 0634 & 62684 & GERLX \\
\hline $\begin{array}{l}P 153 \\
P 154\end{array}$ & $\begin{array}{l}41 \\
41\end{array}$ & $\begin{array}{l}12.19 \\
10.38\end{array}$ & $\begin{array}{l}119 \\
119\end{array}$ & $\begin{array}{l}46.81 \\
50.16\end{array}$ & $\begin{array}{l}5243.0 \\
5121.0\end{array}$ & $\begin{array}{l}979778.49 \\
979784.75\end{array}$ & $\begin{array}{l}-5.1 \\
-7.6\end{array}$ & $\begin{array}{l}0.16 \\
0.11\end{array}$ & $\begin{array}{l}1.5 \\
1.3\end{array}$ & $\begin{array}{l}-183.8 \\
-182.4\end{array}$ & $\begin{array}{l}-16.5 \\
-15.4\end{array}$ & $\begin{array}{l}0634 \\
6644\end{array}$ & $\begin{array}{l}62684 \\
62684\end{array}$ & $\begin{array}{l}\text { GERLK } \\
\text { GERLK }\end{array}$ \\
\hline
\end{tabular}


rable 6--(continued)

ST TION IATITUD LONGITUDE NAIE DLG MIN DLG MIN

$\begin{array}{lllll}P 155 & 41 & 7.80 & 119 & 53.02\end{array}$

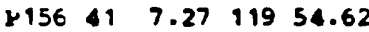
$P 157 \quad 41 \quad 5.70 \quad 11956.29$ $\begin{array}{llllll}P 158 & 41 & 5.05 & 119 & 53.47\end{array}$ $P 15941 \quad 5.16 \quad 119 \quad 52.16$ $\begin{array}{llllll}P 160 & 41 \quad 4.72 \quad 119 & 51.08\end{array}$ $P 16140 \quad 44.26 \quad 119 \quad 10.63$ $\begin{array}{llllll}P 162 \quad 40 & 52.43 \quad 118 & 44.16\end{array}$

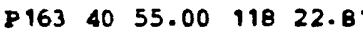

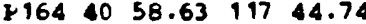

$P 1654056.89 \quad 11759.40$ $\begin{array}{llll}P 166 \quad 40 \quad 57.69 & 117 \quad 59.84\end{array}$ $\begin{array}{lllll}P 167 \quad 40 & 59.58 & 118 & 0.93\end{array}$ $\begin{array}{lllll}P 168 & 41 & 0.12 \quad 118 & 3.12\end{array}$ $\begin{array}{llllll}P 169 & 41 & 1.34 & 118 & 2.41\end{array}$ $\begin{array}{lllll}F 17041 \quad 2.01118 & 0.56\end{array}$ $\begin{array}{llllll}P 171 & 41 & 2.33 & 117 & 59.09\end{array}$ $\begin{array}{lllll}P 172 & 41 \quad 2.02 \quad 117 \quad 56.59\end{array}$ $\begin{array}{lllll}P 173 & 40 & 53.59 & 118 & 27.65\end{array}$ $\begin{array}{lll}P 174 & 40 \quad 56.39 & 118 \quad 29.89\end{array}$

$F 175 \quad 40 \quad 57.98 \quad 118 \quad 30.84$ $176 \quad 41 \quad 0.65 \quad 118 \quad 32.37$ $\begin{array}{llllll}P 177 & 41 & 1.74 & 118 & 33.16\end{array}$ $\begin{array}{lllll}178 & 41 \quad 3.06 & 118 & 33.35\end{array}$ $\begin{array}{lllll}\text { F174 } & 41 & 4.69 & 118 & 32.97\end{array}$ $\begin{array}{llllll}8180 & 41 & 5.55 & 118 & 31.84\end{array}$ $\begin{array}{llllll}P 181 & 41 & 6.31 & 118 & 33.58\end{array}$ $\begin{array}{llllll}P 182 & 41 & 5.03 & 118 & 35.10\end{array}$ $\begin{array}{lllll}P 183 \quad 41 & 6.65 \quad 118 & 35.29\end{array}$ $\begin{array}{lllll}P 184 & 41 & 8.29 & 118 \quad 35.59\end{array}$

$\begin{array}{lllll}P 185 & 41 & 9.04 & 118 & 37.17\end{array}$ $\begin{array}{lllll}P 186 & 41 & 9.04 & 118 & 39.07\end{array}$ $\begin{array}{llllll}P 137 & 41 & 7.64 & 118 & 39.45\end{array}$ $\begin{array}{lllll}P 188 & 41 & 6.43 & 118 & 39.98\end{array}$ $\begin{array}{llllll}P 189 & 41 & 5.38 \quad 118 & 41.05\end{array}$ $\begin{array}{lllll}P 190 & 41 & 4.76 \quad 118 & 38.57\end{array}$ $\begin{array}{lllllll}P 191 & 41 & 3.89 & 118 & 42.49\end{array}$ $\begin{array}{llllll}P 192 & 41 & 2.37 & 118 & 43.33\end{array}$ $\begin{array}{llllll}P 193 & 41 & 0.75 & 118 & 42.94\end{array}$ $P 194 \quad 41 \quad 10.99 \quad 118 \quad 38.38$

$\begin{array}{llll}P 195 \quad 0 \quad 58.82 & 118 \quad 23.00\end{array}$ \begin{tabular}{l}
$\$ 19641 \quad 0.02 \quad 118 \quad 23.34$ \\
\hline 1967
\end{tabular} $\begin{array}{lllll}P & 197 \quad 1.74 \quad 118 \quad 23.06\end{array}$ $\begin{array}{lllll}P & 4198 \quad 3.39 & 118 & 24.43\end{array}$ $P 199 \quad 1 \quad 5.04 \quad 118 \quad 24.91$ $\begin{array}{llllll}P 20 & 41 \quad 6.68 \quad 118 \quad 24.82\end{array}$ $\begin{array}{llllll}P 201 & 11 & B .30 & 118 & 24.79\end{array}$ $\begin{array}{lllll}P 202 & 41 & 10.64 & 118 & 26.54\end{array}$ $P 203 \quad 41 \quad 11.78 \quad 118 \quad 27.27$ $8204411.15 \quad 118 \quad 28.65$
ELEV OBS CRAV TREE TERRAIN DOUG TEET

AIR WUND COMP NWOM
4918.0979794 .41 $4867.0 \quad 979799.44$ 4785.0979807 .13 4699.0979805 .98 4812.0979798 .92 4866.0979792 .22 $3967.0 \quad 979848.56$ 4039.0979848 .50 4163.0979848 .51 4340.6979829 .20

4261.8979839 .61 4383.2979839 .31 4750.7979831 .78 5177.2979806 .44 4731.0979837 .00 4652.2979842 .48 4678.5979833 .86 4383.2979850 .66 4345.0979846 .01 4671.0979830 .74

4753.9979826 .62 $5299.0 \quad 979800.57$ $5550.0 \quad 979793.07$ 5256.0979812 .78 5169.0979824 .57 $5297.0 \quad 979814.99$ $5758.0 \quad 979789.98$ 4888.0979838 .55 4860.0979843 .05 5024.0979836 .92

4254.0979871 .91 $3962.0 \quad 979870.81$ $3988.0 \quad 979866.68$ $3983.0 \quad 979868.84$ 4011.2979868 .63 4259.0979858 .13 4033.7979869 .59 4042.3979869 .15 4053.8979872 .54 $\$ 002.6979872 .66$

4165.0979862 .11 4176.5979865 .16 4207.1979868 .30 4319.2979861 .53 4284.8 979857.06 4194.4979856 .91 4182.8979656 .10 4303.0979851 .84 440.0979847 .09 4611.0979837 .80
$-13.10 .18$

$\begin{array}{ll}-12.1 & 0.18\end{array}$

$-9.8 \quad 0.15$

$\begin{array}{lll}-18.0 & 0.0 f\end{array}$

$\begin{array}{lll}-14.6 & 0.08\end{array}$

$\begin{array}{ll}-15.6 & 0.0 f\end{array}$

$\begin{array}{lll}-13.2 & 0.08\end{array}$

$\begin{array}{ll}-18.7 & 0.0 f\end{array}$

$\begin{array}{ll}-10.9 & 0.08\end{array}$

$\begin{array}{lll}-18.9 & 0.18\end{array}$

$\begin{array}{ll}-13.30 .08 & 0.0\end{array}$

$-3.40 .08$

20.80 .45

$34.7 \quad 0.1 f$

$21.5 \quad 0.18$

18.60 .18

$12.0 \quad 0.18$

1.50 .18

$5.8 \quad 0.18$

$17.0 \quad 0.08$

18.30 .18

$\begin{array}{lll}39.5 & 0.2\end{array}$

$54.0 \quad 0.18$

$44.1 \quad 0.28$

$45.3 \quad 0.11$

46.40 .1

$63.6 \quad 0.41$

$\begin{array}{lll}32.3 & 0.18\end{array}$

$\begin{array}{lll}31.8 & 0.18\end{array}$

$38.6 \quad 0.5$

0.10 .15

$-28.40 .0 f$

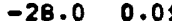

$\begin{array}{lll}-24.5 & 0.08\end{array}$

$-20.5$

$-6.8$

$-15.2$

$-12.6$

$-5.7$

$-25.7$

$-2$.

$0.080 .2-146.0$

$\begin{array}{lllll}-0.5 & 0.08 & 0.2 & -144.0\end{array}$

$\begin{array}{lll}3.0 & 0.08 & 0.2-141.6\end{array}$

$4.30 .180 .2-144.1$

$\begin{array}{llll}-5.9 & 0.06 & 0.2 & -153.1\end{array}$

$\begin{array}{llll}-17.0 & 0.08 & 0.2 & -161.1\end{array}$

$\begin{array}{llll}-21.3 & 0.08 & 0.3 & -165.0\end{array}$

$\begin{array}{llll}-17.8 & 0.08 & 0.8 & -165.0\end{array}$

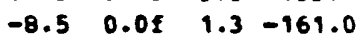

$\begin{array}{llll}-3.6 & 0.15 & 1.5 & -160.7\end{array}$
IBOS ACC DATE BASE NWOY CODE M-D-Y NAME

-14.3663462684 GLRLX -11.6 C644 62684 GERLX -6.2063462684 GERIX $-12.7 \quad 663362684$ GERLX -13.3 G534 62684 GLPIK -16.4653462684 GERLX $3.5 \times 12462784$ GERIX -4.9812362984 WINN 0.1653371284 WINNA -6.66533101484 WINlin

-2.4653462784 WINNA 3.26534102784 WIN:iA 14.9074462784 WINivn 14.2643462784 WIN!ih 15.5 G434 62784 WINN $15.5 \quad 673462784$ WINTIA 8.3643462784 WINMI B.5 G534 62784 WINTin 10.7 G534 62884 WINih 10.2663462884 WINTin

8.7653462884 WINNh $11.6 \quad 663462884 \mathrm{bzlilis}$ $17.7 \quad 663462884$ bistwin 17.9664462884 WIINLA 22.0664462884 wiNin 19.2664462884 WINNi $22.66644^{\circ} 62884$ WINiin $18.7 \quad 6634 \quad 62884$ wIilin 20.1663462884 iillivi 23.2663462884 WiNk

$9.76634 \quad 62884$ WINN $-9.8 \quad 663362984$ WIANin -10.9663462884 WINivo -7.5665462884 wiNivi $-4.9 \times 12462884$ WINii

0.7 GE34 62884 WINh h -0.6 N124 62884 WIINh

1.4 N124 62884 WINIS 7.7 N124 62884 WINNh -7.7 V123 71284 WINNA

B. 1 G534 71184 WINTih 10.0 N124 79184 WINTIA 12.4 N124 71184 WINNA 9.9 N124 71184 WINNA 1.0 N124 71184 WINNA -6.8 N124 71184 WINNA -10.5 N124 71284 WINNA -10.3663471184 WINNA $-6.0 \quad 6633 \quad 71284$ WIMNA -5.9063471184 WINBí 
Table 6--(continued)

GTATION LATITUDE LONGITUDE NHML DEG MIN DES MIN

Exv

OUS GRAV

TREE

TLRRAIN BOUG

ISOS ACC DATE BASE

\begin{tabular}{l}
$\$ 20549 \quad 9.84 \quad 118 \quad 25.05$ \\
\hline
\end{tabular} $\begin{array}{llllll}8206 & 41 & 11.30 & 118 & 22.94\end{array}$ $\begin{array}{lllll}8207 \quad 41 & 12.48 & 118 & 21.44\end{array}$ $\begin{array}{llllll}P 208 & 41 & 13.96 & 118 & 19.54\end{array}$ $\begin{array}{lllll}P 209 & 41 & 15.18 & 118 \quad 18.41\end{array}$ $\begin{array}{llllll}\text { P2 } 10 & 41 & 16.56 & 118 & 17.73\end{array}$ $\begin{array}{lllll}P 211 & 41 & 19.30 & 118 & 15.77\end{array}$ $\begin{array}{lll}P 212 & 429.64118 \quad 15.56\end{array}$ $\begin{array}{llllll}P 213 \quad 41 & 8.42 \quad 118 & 32.81\end{array}$ $\begin{array}{lllll}P 214 & 41 \quad 8.23 \quad 118 & 30.94\end{array}$

4206.2979859 .77 4166.6979870 .01 $\$ 196.3979870 .64$ 4198.7979878 .15 4208.0979879 .08 4182.0979889 .53 $\$ 188.0979891 .90$ 4195.0979890 .64 $6070.0 \quad 979773.77$ $5088.0 \quad 979829.65$

$\begin{array}{lllll}P 215 \quad 41 \quad 8.44 \quad 118 & 29.52\end{array}$ $\begin{array}{lllll}P 216 & 41 & 8.26 & 118 & 28.67\end{array}$ $\begin{array}{llllll}P 217 & 41 & 8.18 & 118 & 27.05\end{array}$ $\begin{array}{lllll}\text { P2 } 18 & 41 & 7.89 & 118 & 26.02\end{array}$ $\begin{array}{lllll}P 219 & 41 & 13.06 \quad 118 \quad 28.17\end{array}$ $\begin{array}{lllll}P 220 & 41 & 14.24 & 118 \quad 28.40\end{array}$ $\begin{array}{lllll}P 229 & 41 & 17.32 & 118 & 27.52\end{array}$ $\begin{array}{lllll}P 222 & 41 & 18.56 \quad 118 \quad 29.42\end{array}$ P223 $41 \quad 21.60 \quad 118 \quad 31.91$ $\begin{array}{lllll}P 224 & 41 & 19.98 & 118 \quad 33.02\end{array}$

$\begin{array}{lllll}P 225 & 41 & 18.30 & 118 & 33.17\end{array}$ $\begin{array}{lllll}\text { P226 } & 41 & 16.70 & 118 & 34.09\end{array}$ $\begin{array}{llllll}P 227 & 41 & 14.56 \quad 118 & 36.15\end{array}$ $\begin{array}{lllll}P 228 & 41 & 13.37 & 118 & 36.96\end{array}$ $\begin{array}{llllll}P 229 & 41 & 57.10 & 118 & 37.49\end{array}$ $\begin{array}{llllll}P 282 & 41 & 33.43 & 119 & 59.87\end{array}$ $P 2834136.44 \quad 11957.42$
$P 284$ $\begin{array}{lllll}P 284 & 41 & 36.28 & 119 & 56.38\end{array}$ $\begin{array}{lllll}P 285 & 41 & 34.87 & 119 & 52.39\end{array}$

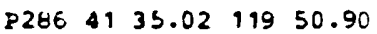

$P 287 \quad 4 \quad 35.42 \quad 119 \quad 37.77$ $\begin{array}{lllll}P 288 & 4137.25 \quad 119 \quad 33.81\end{array}$ \begin{tabular}{l}
$P 289 \quad 4 \quad 38.71 \quad 11932.61$ \\
\hline
\end{tabular} $\begin{array}{lllll}P 290 & 41 & 38.85 & 119 & 35.10\end{array}$ $P 291 \quad 4140.10 \quad 11934.59$ P292 $41 \quad 41.48 \quad 11934.55$ \begin{tabular}{l}
$P 29341 \quad 42.87 \quad 11934.38$ \\
\hline
\end{tabular} $P 2544142.1911935 .30$

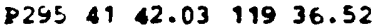
$\begin{array}{lllll}P 296 & 41 \quad 37.18 & 119 & 36.97\end{array}$

$P 297 \quad 4131.92 \quad 119 \quad 29.81$
$P 29841$ $\begin{array}{llllll}P 296 & 41 \quad 29.89 & 119 & 28.70\end{array}$ $\begin{array}{lllll}P 299 & 41 & 28.69 & 119 & 28.50\end{array}$ $8300 \quad 4128.4111927 .05$
8301 $\begin{array}{lllll}830141 & 27.56 \quad 119 & 26.37\end{array}$

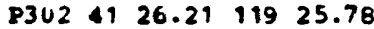
$P 303 \quad 1 \quad 24.97 \quad 11925.36$ $P 3044123.56 \quad 11924.82$
830541 $83054122.03 \quad 11923.46$

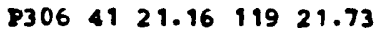

4607.0979846 .61 $4439.0 \quad 979848.50$ 4248.0979851 .84 4206.0979851 .52 4771.0979828 .64 $5052.0 \quad 979815.75$ 6038.0979771 .88 $5054.0 \quad 979835.68$ 4056.4979895 .25 4014.4979894 .50

4027.5979900 .93 4006.1979893 .61 3998.0979892 .84 4007.7979891 .33 4218.0979925 .52 4863.8979833 .71 5724.0979786 .40 $5759.0 \quad 979787.50$ $5960.8 \quad 979774.39$ $5541.0 \quad 979793.87$

5680.6979790 .33 $5716.0 \quad 979790.20$ $5773.0 \quad 979785.64$ $5628.0 \quad 979795.08$ $5624.0 \quad 979794.76$ 5624.0979801 .91 $5707.0 \quad 979796.26$ 5633.0979801 .03 5827.0979780 .54 $5622.0 \quad 979798.06$

$6029.0 \quad 979758.77$ $5899.0 \quad 979762.26$ 5595.2979776 .98 5507.5979781 .55 5453.0979781 .86 5326.8979783 .45 5202.5979790 .96 5157.2979796 .67 5075.7979797 .85 5008.9979805 .84

\section{AIK HND COMP NWOM}

$\begin{array}{llll}-17.7 & 0.0 f & 0.5 & -162.0 \\ -13.4 & 0.0 f & 0.7 & -156.1 \\ -11.7 & 0.0 f & 1.0 & -155.1 \\ -6.2 & 0.16 & 1.1 & -149.6 \\ -6.2 & 0.08 & 1.0 & -150.0 \\ -0.3 & 0.06 & 1.1 & -143.1 \\ -1.5 & 0.06 & 0.5 & -145.1 \\ -5.6 & 0.06 & 0.5 & -149.5 \\ 73.6 & 1.4 f & 6.2 & -128.7 \\ 37.5 & 0.8 f & 2.5 & -135.0\end{array}$

8.9 $0.1 f \quad 1.1-148.5$

$-4.8$ $\begin{array}{llll}-19.2 & 0.05 & 0.5 & -164.9\end{array}$ $\begin{array}{llll}-23.1 & 0.0 \text { f } & 0.3 & -167.5\end{array}$

$\begin{array}{lllll}-0.6 & 0.15 & 2.0 & -162.7\end{array}$

$\begin{array}{llll}11.2 & 0.16 \quad 2.5 & -160.0\end{array}$

$55.4 \quad 0.5 f \quad 4.3 \quad-147.8$

$24.8 \quad 1.2 f \quad 6.8 \quad-142.1$

$\begin{array}{llll}-13.9 & 0.16 & 2.9 & -150.6\end{array}$

$\begin{array}{llll}-16.2 & 0.1 f \quad 2.2 & -152.2\end{array}$

$\begin{array}{llll}-6.0 & 0.1 f \quad 3.0 & -141.6\end{array}$ $\begin{array}{lllll}-12.9 & 0.05 & 3.4 & -147.4\end{array}$

$\begin{array}{lllll}-11.3 & 0.16 & 3.2 & -145.7\end{array}$

$\begin{array}{lllll}-10.1 & 0.0 f & 2.6 & -145.4\end{array}$

$\begin{array}{llll}-21.6 & 0.0 f & 0.9 & -165.9\end{array}$

$\begin{array}{lllll}-17.3 & 0.1 f & 1.3 & -183.3\end{array}$

$\begin{array}{lllll}11.8 & 0.1 f \quad 1.1 & -183.8\end{array}$

$\begin{array}{lllll}16.4 & 0.36 & 1.7 & -179.8\end{array}$

$24.4 \quad 0.26 \quad 1.6 \quad-178.9$

$\begin{array}{lllll}4.2 & 0.06 & 0.8 & -185.5\end{array}$

$13.1 \quad 0.05 \quad 0.4-181.6$

$\begin{array}{lllll}13.6 & 0.05 & 0.5 & -182.3\end{array}$

$12.20 .0 f \quad 0.6-185.6$

$\begin{array}{lllll}7.8 & 0.1 f & 0.6 & -185.0\end{array}$

$\begin{array}{lllll}5.3 & 0.0 f & 0.5 & -187.5\end{array}$

$\begin{array}{llll}10.3 & 0.0 f & 0.6 & -182.4\end{array}$

$\begin{array}{llll}10.4 & 0.1 f & 0.5 & -185.2\end{array}$

$\begin{array}{lllll}9.2 & 0.2 f & 0.7 & -183.7\end{array}$

$\begin{array}{lllll}7.2 & 0.1 f & 0.5 & -192.5\end{array}$

$\begin{array}{llll}12.7 & 0.05 & 0.4 & -180.1\end{array}$

$19.6 \quad 0.16$

$13.9 \quad 0.38$

$0.8-186.8$

$\begin{array}{llll}1.5 & 0.15 & 0.7 & -190.0\end{array}$

$\begin{array}{llll}-1.4 & 0.1 f & 0.6 & -190.1\end{array}$

$\begin{array}{llll}-4.9 & 0.26 & 0.7 & -191.6\end{array}$

$\begin{array}{llll}-13.2 & 1.9 f \quad 2.8 & -193.5\end{array}$

$-15.5 \quad 1.96 \quad 3.3-191.1$

$\begin{array}{llll}-11.9 & 1.2 f & 2.7 & -186.6\end{array}$

$-16.12 .163 .6-187.0$

$\begin{array}{llll}-13.1 & 1.96 \quad 3.6 & -181.8\end{array}$
NOA CODE $M-D-Y$ NAME

$-7.3 \times 12471184$ WINNA -1.1 N124 71184 WINN 0.0 N124 71184 WINNA 5.8 N124 71.184 WINIS 5.6064471184 WINNA 12.7663471184 WINKA 11.1663471184 WINNA 7.1663471184 WIINNA 25.1664471284 WINNA 19.1663471284 WINNA

5.8675471284 WINNA $-2.3 G 75471284$ WINNA -10.5663471284 WINNA -13.1 G624 71284 WINNis -7.7663471284 WINNh -4.8675471284 WINiNh 7.8663471284 WINNA 13.9663471284 WINlvi 6.4 N124 71284 WINNis 4.5 D224 71284 WINTis

14.7 N 12471284 WINNA 8.5 N124 71284 WIN?JA 9.8664471284 WINLIA 9.8 N124 71284 WINKin $-1.3 C 632101684$ DENIO -16.9 V224 $8785 \mathrm{CH} 15$ -17.26634 B $785 \mathrm{CH} 15$ $-13.1 \mathrm{C} 644$ \& $785 \mathrm{CH} 15$ $-11.3 \times 224$ \& $785 \mathrm{CH} 15$ -17.4 U342 $82785 \mathrm{Ch} 15$

-11.6 N124 8 $785 \mathrm{CH} 15$ $-11.86644 \quad 8785 \mathrm{CH} 15$ $-14.9 \mathrm{C634} 8785 \mathrm{CH} 15$ $-14.5 \mathrm{C644} 8785 \mathrm{CH} 15$ $-17.0 \mathrm{C644} 8785 \mathrm{CH} 15$ $-11.76633 \quad 8785 \mathrm{CH} 15$ $-14.6 \mathrm{C} 634 \mathrm{~B} 785 \mathrm{CH} 15$

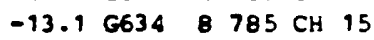
$-22.1 \mathrm{C6} 44 \quad 8785 \mathrm{CH} 15$

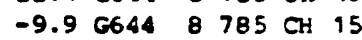

$-16.86633$ $-18.06534$

$-20.4 B 123$

$-20.6 \times 124$

$-22.3 \mathrm{~B} 123$

$-24.4 N 224$

$-22.4 \times 224$

$-18.1 N 224$

$-19.2 \times 124$

$-14.4 N 224$
$8885 \mathrm{CH} 15$ $8885 \mathrm{CH} 15$ $81085 \mathrm{CH} 15$ $8885 \mathrm{CH} 15$ $81085 \mathrm{CH} 15$ $8885 \mathrm{CH} 15$ $8 \mathrm{B85} \mathrm{CH} 15$ $8885 \mathrm{CH} 15$ (685 $\mathrm{CH} 15$ C $885 \mathrm{CH} 15$ 
Table 6-(continued)

STATION LATITUDE LONGITUDE NAME DLE MIN DEG MIN

$P 307 \quad 1 \quad 19.89 \quad 11920.36$ P308 $4136.07 \quad 11933.88$ P309 $4136.07 \quad 11931.93$ P3 $104134.72 \quad 11931.88$ $\begin{array}{llllll}P 311 & 41 & 33.67 & 119 & 29.36\end{array}$ $\begin{array}{llllll}P 312 & 41 & 32.30 & 119 & 27.88\end{array}$ $\begin{array}{llllll}P 313 \quad 4130.57 & 119 & 26.48\end{array}$ P314 $4130.66 \quad 11925.31$ $\begin{array}{lllll}P 315 & 4130.12 \quad 11924.56\end{array}$ $\begin{array}{lll}\text { P316 } & 1130.07 \quad 11923.44\end{array}$

P317 $4130.62 \quad 11922.67$ $\begin{array}{lllll}P 318 & 4130.80 \quad 119 & 21.71\end{array}$ $\begin{array}{llllll}P 319 & 41 & 31.98 & 119 & 20.90\end{array}$ P320 $4132.08 \quad 119 \quad 19.32$ $\begin{array}{llllll}\text { P321 } 4130.76 \quad 119 & 18.38\end{array}$ $\begin{array}{lllll}\text { P322 } 41 & 29.38 & 119 & 18.00\end{array}$ $\begin{array}{lllll}P 323 & 41 & 27.91 & 119 & 17.81\end{array}$ P324 $4126.41 \quad 119 \quad 16.89$ P325 $4137.56 \quad 11930.45$ $\begin{array}{lllll}\text { P326 } & 41 & 27.63 & 119 & 24.85\end{array}$

$\begin{array}{lllll}P 327 \quad 41 & 27.10 \quad 119 & 23.00\end{array}$ $\begin{array}{lllll}\text { P328 } & 41 & 27.82 & 119 & 23.79\end{array}$ $\begin{array}{lllll}\text { P329 } 4131.98 & 119 & 32.35\end{array}$ $\begin{array}{lllll}\text { P330 } 4130.18 & 119 & 30.98\end{array}$ $\begin{array}{lllll}P 331 & 41 & 28.91 & 11933.08\end{array}$ $\begin{array}{lllll}\text { P332 } 41 \quad 27.17 \quad 119 & 34.78\end{array}$ $\begin{array}{llllll}\text { P333 } 4127.11 & 11933.22\end{array}$

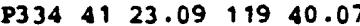
$\begin{array}{lllll}\text { P335 } 41 \quad 24.39 & 119 & 41.63\end{array}$ P336 $4123.87 \quad 11932.00$

$\begin{array}{lllll}P 337 \quad 4123.83 & 119 & 30.10\end{array}$ $\begin{array}{llllll}\text { P338 } 4124.01 & 119 & 29.00\end{array}$ $\begin{array}{lllll}P 339 & 41 & 24.16 \quad 119 & 27.66\end{array}$ $\begin{array}{llllll}P 340 & 41 & 23.58 & 119 & 26.61\end{array}$ $\begin{array}{lllll}\text { P341 } 41 & 22.98 & 119 & 26.95\end{array}$ $\begin{array}{lllll}P 342 & 11 & 21.32 & 119 & 26.11\end{array}$ P343 $4120.03 \quad 11926.48$ P344 $41 \quad 19.78 \quad 11924.65$ $\begin{array}{lllll}P 345 \quad 41 & 19.43 \quad 119 & 22.99\end{array}$ P346 $41 \quad 16.92 \quad 119 \quad 22.47$

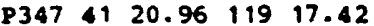
$\begin{array}{lllll}\text { P348 } 4122.85 \quad 119 & 30.47\end{array}$ $\begin{array}{lllll}\text { P349 } 4122.00 \quad 119 & 29.79\end{array}$ $\begin{array}{llllll}8350 \quad 41 & 20.61 & 119 & 30.45\end{array}$ $\begin{array}{llllll}P 351 & 41 & 19.16 & 119 & 30.27\end{array}$ $\begin{array}{llllll}\text { P3 } 52 & 41 & 18.96 & 119 & 28.33\end{array}$ $\begin{array}{lllll}P 353 \quad 41 & 18.48 & 119 & 26.56\end{array}$ $\begin{array}{lllll}P 354 & 41 & 16.95 & 119 & 25.54\end{array}$ $\begin{array}{lllll}\text { P355 } 41 \quad 17.47 \quad 119 & 30.30\end{array}$ $\begin{array}{lllll}\text { P3 } 56 \quad 41 & 17.90 \quad 119 \quad 32.43\end{array}$
EILV OAS GRAV FEET MGAL

4952.0979814 .00 5759.9979785 .26 5813.0979781 .45 5936.0979769 .97 6399.0979741 .16 6370.0979739 .59 5682.1979774 .64 5527.2979783 .79 5505.3979779 .13 5486.2979780 .71

5494.5979778 .17 5516.9979775 .38 5474.5979781 .68 $5567.0 \quad 979776.29$ 5614.0979770 .85 $5693.0 \quad 979762.89$ 5765.0979761 .17 $5954.0 \quad 979745.63$ $5892.0 \quad 979779.86$ 5424.0979781 .82

$5344.0 \quad 979784.73$ 5347.0979785 .64 6210.2979740 .98 5904.8979760 .84 6187.0979739 .43 6011.0979748 .06 5808.6979763 .64 $5686.0 \quad 979766.79$ 5704.9979769 .70 $5811.0 \quad 979762.15$

$5741.0 \quad 979763.68$ $5792.0 \quad 979761.83$ $5801.0 \quad 979759.37$ 5498.0979774 .62 $5449.0 \quad 979777.81$ $5842.0 \quad 979753.40$ $5844.0 \quad 979757.63$ 5851.0979756 .93 5638.0979770 .53 5786.0979762 .49

5702.0979769 .11 5801.0979761 .76 5757.0979759 .69 5819.0979759 .49 6131.0979734 .12 6194.0979736 .01 5949.0979749 .53 6233.0979732 .29 6062.0979742 .58 6545.0979708 .20
FREE TERRAIN BOUG AIR HAND COMP ANOM

$\begin{array}{rlllll}-8.4 & 3.6 f & 5.9 & -172.9 & -6.2 & 0444 \\ 14.6 & 0.0 f & 0.4 & -182.9 & -12.5 & 8124 \\ 15.7 & 0.1 f & 0.6 & -183.4 & -12.9 & 0333 \\ 17.8 & 0.1 f & 0.6 & -185.5 & -15.3 & 6634 \\ 34.1 & 0.1 f & 1.0 & -184.6 & -14.5 & 6634 \\ 31.9 & 0.4 f & 1.7 & -185.2 & -15.3 & 6634 \\ 4.9 & 0.1 f & 0.6 & -189.8 & -19.9 & N 124 \\ -0.7 & 0.1 f & 0.6 & -190.0 & -20.1 & N 124 \\ -6.6 & 0.0 f & 0.5 & -195.4 & -25.6 & N 124 \\ -6.7 & 0.0 f & 0.4 & -194.9 & -25.2 & N 124\end{array}$

$-9.30 .16$

$\begin{array}{lll}-10.3 & 0.08\end{array}$

$\begin{array}{lll}-9.7 & 0.0 f\end{array}$

$\begin{array}{lll}-6.6 & 0.0 f\end{array}$

$0.5-197.6$

$-27.9 \times 124$

$-29.7 N 124$

$-27.4 N 124$

$\begin{array}{lll}-27.5 & 6634\end{array}$

$\begin{array}{lllllll}-5.6 & 0.0 f & 0.6 & -197.9 & -28.6 & 6633\end{array}$

$\begin{array}{lllllll}-4.1 & 0.16 & 0.8 & -199.0 & -30.1 & 6634\end{array}$

$3.2 \quad 0.1 f$

$\begin{array}{lll}7.6 & 0.31\end{array}$

$19.3 \quad 0.16$

$0.7-194.2$

$1.2-195.7$

$-25.8 \quad 6634$

$-27.96634$

$-11.86634$

$\begin{array}{lllllll}-7.8 & 0.1 f & 0.6 & -193.7 & -24.4 & 6634\end{array}$

$\begin{array}{ll}-11.6 & 0.2 f\end{array}$

$\begin{array}{lll}-11.5 & 0.06\end{array}$

$0.9-194.5$

$-25.5 \quad 6644$

$\begin{array}{lllllll}18.7 & 0.1 f & 0.8 & -193.8 & -23.8 & N 224\end{array}$

$\begin{array}{lllllll}12.6 & 0.4 f & 1.3 & -189.0 & -19.2 & \text { N224 }\end{array}$

$\begin{array}{lllllll}19.6 & 0.1 f & 0.9 & -192.1 & -22.5 & 6634\end{array}$

$14.3 \quad 0.2 f \quad 0.9-191.3 \quad-21.9 \quad 6644$

$10.9 \quad 0.1 f \quad 0.7-188.0 \quad-18.5 \quad N 124$

$8.60 .0 f \quad 0.5-186.4 \quad-17.5$ is 124

$\begin{array}{llllll}11.3 & 0.1 f & 0.5 & -184.3 & -15.3 & N 123\end{array}$

$\begin{array}{llllll}14.5 & 0.0 f & 0.5 & -184.7 & -15.8 & 6633\end{array}$

9.50 .16

$0.5-187.3-18.5 \quad 6634$

$\begin{array}{lllllll}12.2 & 0.2 f & 0.7 & -186.2 & -17.5 & 6634\end{array}$

$\begin{array}{lllllll}10.4 & 0.1 f & 0.6 & -188.4 & -19.7 & 6634\end{array}$

$\begin{array}{lllllll}-2.0 & 0.2 f & 0.9 & -190.1 & -21.6 & 6634\end{array}$

$\begin{array}{lllllll}-2.5 & 0.5 f & 1.3 & -188.6 & -20.1 & 6634\end{array}$

$\begin{array}{lllllll}12.5 & 0.5 f & 1.2 & -187.1 & -19.3 & 6644\end{array}$

$\begin{array}{lllllll}18.8 & 0.0 f & 0.6 & -181.3 & -13.9 & 6644\end{array}$

$\begin{array}{lllllll}19.2 & 0.5 f & 1.3 & -180.6 & -13.5 & 6644\end{array}$

$\begin{array}{llllll}13.3 & 0.3 f & 0.9 & -179.6 & -12.8 & 6644\end{array}$

$\begin{array}{lllllll}22.9 & 0.1 f & 0.9 & -175.1 & -9.2 & 6644\end{array}$

$\begin{array}{llllll}15.6 & 0.06 & 1.4 & -178.9 & -12.7 & 6644\end{array}$

$14.7 \quad 0.16 \quad 0.6 \quad-184.0 \quad-15.5 \quad 6634$

$\begin{array}{llllll}9.8 & 0.56 & 1.0 & -187.0 & -18.6 & 6644\end{array}$

$\begin{array}{llllll}17.5 & 0.2 f & 0.9 & -181.6 & -13.4 & 0734\end{array}$

$\begin{array}{lllllll}23.6 & 0.2 f & 1.0 & -186.0 & -18.4 & 6644\end{array}$

$\begin{array}{llllll}31.7 & 0.1 f & 1.0 & -180.0 & -12.7 & 6644\end{array}$

$\begin{array}{llllllll}22.9 & 0.3 f & 1.0 & -180.4 & -13.4 & 6644\end{array}$

$\begin{array}{llllll}34.7 & 0.5 f & 2.0 & -177.4 & -11.1 & 6644\end{array}$

$\begin{array}{llllll}28.1 & 0.1 f & 1.0 & -179.2 & -11.9 & 6634\end{array}$

$\begin{array}{lllllll}38.5 & 0.1 f & 1.7 & -184.6 & -17.2 & 6634\end{array}$
DATE BASE

M-D-Y NAME

$8885 \mathrm{CH} 15$ $8985 \mathrm{CH} 15$ 81685 CH 15 $8985 \mathrm{CH} 15$ $8985 \mathrm{CH} 15$ $8985 \mathrm{CH} 15$ $8985 \mathrm{CH} 15$ $8985 \mathrm{CH} 15$ $8985 \mathrm{CH} 15$ $8985 \mathrm{CH} 15$

$8985 \mathrm{CH} 15$ 8985 CH 15 $8985 \mathrm{CH} 15$ $8985 \mathrm{CH} 15$ $8985 \mathrm{CH} 15$ $8985 \mathrm{CH} 15$ B $985 \mathrm{CH} 15$ $8985 \mathrm{CH} 15$ $8985 \mathrm{CH} 15$ $81085 \mathrm{CH} 15$

$81085 \mathrm{CH} 15$ $81085 \mathrm{CH} 15$ $81085 \mathrm{CH} 15$ $81085 \mathrm{CH} 15$ $81085 \mathrm{CH} 15$ $81085 \mathrm{CH} 15$ $81085 \mathrm{CH} 15$ $81085 \mathrm{CH} 15$ $82685 \mathrm{CH} 15$ $81285 \mathrm{CH} 15$

$81185 \mathrm{CH} 15$ $81185 \mathrm{CH} 15$ $81185 \mathrm{CH} 15$ $81185 \mathrm{CH} 15$ $81185 \mathrm{CH} 15$ $81185 \mathrm{CH} 15$ $81185 \mathrm{CH} 15$ $81185 \mathrm{CH} 15$ $81185 \mathrm{CH} 15$ $81185 \mathrm{CH} 15$

$81185 \mathrm{CH} 15$ $81285 \mathrm{CH} 15$ $81285 \mathrm{CH} 15$ $81285 \mathrm{CH} 15$ $81285 \mathrm{CH} 15$ $81285 \mathrm{CH} 15$ $81285 \mathrm{CH} 15$ $81285 \mathrm{CH} 15$ $81285 \mathrm{CH} 15$ 81285 CH 15 
rable 6-(continued)

STATION IATITUDE LONGITUDE WWE LLG MIN UDG MIN

$\begin{array}{lllll}8357 \quad 41 & 16.38 & 119 & 30.46\end{array}$ $\begin{array}{llllll}P 358 & 41 & 15.39 & 119 & 29.64\end{array}$ $\begin{array}{llllll}P 359 & 41 & 14.53 & 119 & 28.50\end{array}$ $\begin{array}{llllll}P 360 & 41 & 21.31 & 119 & 36.48\end{array}$ $\begin{array}{lllll}P 361 & 41 & 14.82 & 119 & 32.25\end{array}$ \begin{tabular}{l} 
P362 $4114.17 \quad 11931.22$ \\
\hline 36341
\end{tabular} \begin{tabular}{l}
$8363 \quad 41 \quad 13.83 \quad 119 \quad 29.95$ \\
\hline
\end{tabular} $8364 \quad 4112.39119 \quad 23.91$ $\begin{array}{lllll}P 365 & 41 & 13.03 \quad 119 & 23.42\end{array}$ $\begin{array}{lll}P 366 & 41 & 13.85 \quad 119 \quad 27.00\end{array}$

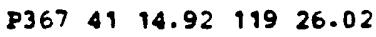
$\begin{array}{llllll}P 368 & 41 & 19.59 & 119 \quad 32.38\end{array}$ $\begin{array}{lllll}P 369 & 41 \quad 19.92 \quad 119 \quad 33.58\end{array}$ P370 $4136.30 \quad 11936.28$ $P 314138.4711938 .85$

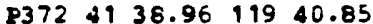
$\begin{array}{lllll}P 373 \quad 41 & 35.95 & 119 & 42.56\end{array}$ $8374 \quad 4137.00 \quad 11941.06$ $\begin{array}{lllll}P 375 & 41 & 36.72 & 119 & 43.89\end{array}$ $8376 \quad 4138.26 \quad 119 \quad 46.02$

$83774139.31 \quad 11948.64$ $\begin{array}{lllll}2378 & 41 & 38.68 & 119 & 49.77\end{array}$

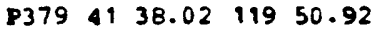

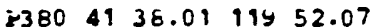

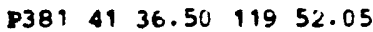

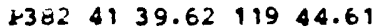
$\begin{array}{lllllll}83 & 41 & 40.20 & 119 & 42.77\end{array}$ $P==4 \quad 41 \quad 41.08 \quad 119 \quad 42.90$ $\begin{array}{llllll}P 385 & 41 \quad 40.04 \quad 119 & 47.96\end{array}$

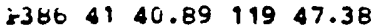

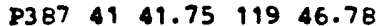
$\begin{array}{llllll}5388 & 41 & 42.53 & 119 & 46.04\end{array}$ $\begin{array}{lllllll}8389 & 41 & 43.86 & 119 & 46.82\end{array}$ $\begin{array}{llllll}\text { P390 } 41 & 43.26 & 199 & 44.98\end{array}$ $\begin{array}{llllll}2391 & 41 & 4.65 & 119 & 43.11\end{array}$ P392 $4145.49 \quad 11942.46$

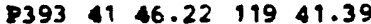

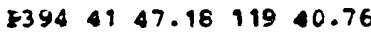
$\begin{array}{lllllll}\text { P395 } 41 & 48.20 & 119 & 40.44\end{array}$

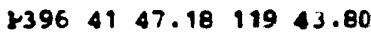

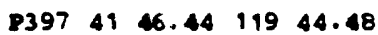

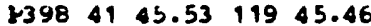
$\begin{array}{lllll}2399 & 41 \quad 45.56 \quad 119 \quad 46.61\end{array}$

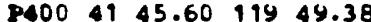
$840141 \quad 46.20 \quad 11951.40$ PQU2 4145.1111952 .04 $P 403 \quad 41 \quad 43.38 \quad 11952.05$
$P 404$

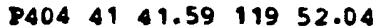

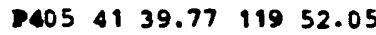
$2406 \quad 4134.98 \quad 119 \quad 49.32$
IIEV DBS GRAV TREL TERRAIN DOUG FEET MLAL

5802.0979758 .65 5422.0979782 .22 $5294.0 \quad 979785.18$ 5815.6979756 .88 5495.5979773 .57 5383.7979774 .49 5338.3979775 .36 $6224.0 \quad 979727.94$ 6035.0979740 .35 5374.0979779 .00

5260.0979790 .52 6010.0979740 .48 5884.0979750 .56 5616.0979795 .41 5625.0979793 .93 $5842.0 \quad 979780.47$ $5683.0 \quad 979790.35$ 5615.0979793 .29 5648.0979792 .53 $5576.0 \quad 979796.12$

5526.2979802 .99 5525.0979802 .81 5546.3979802 .10 $5613.0 \quad 979795.21$ $5574.0 \quad 979798.35$ $5983.0 \quad 979773.86$ 6097.0979766 .23 $6356.0 \quad 979747.45$ 5524.8979803 .98 5523.9979803 .86

5526.4979805 .52 5533.7979804 .58 5531.0979806 .50 5589.3979799 .62 5528.8979804 .66 5536.3979807 .88 5529.9979810 .77 5530.0979813 .64 5584.2979814 .48 5546.0979813 .81

$5543.0 \quad 979813.59$ 5532.0979811 .52 $5578.0 \quad 979809.18$ 5524.0979611 .41 5534.0979812 .64 5545.7979808 .72 5530.4979805 .97 $5532.0 \quad 979403.46$ $5528.0 \quad 979800.34$ 5538.7979794 .12

AIR WAND COMP NHON

\begin{tabular}{|c|c|c|c|c|c|c|c|c|}
\hline & & & & & & & & \\
\hline 0.7 & $.2 E$ & .0 & -174.7 & -7.8 & 6644 & 1285 & $\mathrm{CH}$ & \\
\hline .9 & $1 f$ & .6 & -178.4 & 11.9 & 6634 & 1285 & $\mathrm{CH}$ & \\
\hline 3.5 & $0 f$ & .6 & 185.8 & 17.2 & & 785 & $\mathrm{CH}$ & \\
\hline .8 & f & .6 & -178.4 & 11.4 & & 1385 & $\mathrm{CH}$ & \\
\hline .2 & & .6 & -183.3 & 16.5 & & 385 & $\mathrm{CH}$ & \\
\hline 1. & & 5 & 84.7 & -18.2 & 24 & 185 & $\mathrm{CH}$ & \\
\hline .3 & & 5 & 16.1 & & & & $\mathrm{CH}$ & \\
\hline .0 & & 3 & & & & & $\mathrm{CH}$ & \\
\hline .3 & & .7 & & & & & & \\
\hline 4.5 & & .0 & 15.4 & 9.1 & & & & \\
\hline 7.9 & .18 & .9 & 37.7 & -19.7 & 6634 & & $\mathrm{CH}$ & 15 \\
\hline 5.7 & & .7 & 5.8 & .6 & & & $\mathrm{CH}$ & 15 \\
\hline 10.8 & $.0 f$ & 0.4 & 81.9 & .8 & 6634 & & $\mathrm{Ch}$ & 15 \\
\hline 7.0 & $0 f$ & .4 & 85.9 & .9 & 6643 & & $\mathrm{CH}$ & 15 \\
\hline 7.9 & & .5 & -184.1 & .3 & 6654 & & $\mathrm{CH}$ & 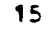 \\
\hline 12.6 & $.0 f$ & .4 & -182.3 & -12.8 & 6634 & & $\mathrm{CH}$ & 15 \\
\hline 7.6 & 16 & 0.5 & 84.9 & -15.1 & 16644 & & $\mathrm{CH}$ & 15 \\
\hline 10.3 & $.0 f$ & .4 & 83.4 & -14.0 & 6634 & & $\mathrm{CH}$ & 15 \\
\hline 6.9 & & & & & & & $\mathrm{CH}$ & 15 \\
\hline 5. & & 4 & & & & & & \\
\hline .1 & & .4 & & & & & $\mathrm{CH}$ & . \\
\hline 8.4 & & .5 & & .6 & & & $\mathrm{CH}$ & \\
\hline 7.8 & & .8 & & & & & $\mathrm{CH}$ & \\
\hline 9.5 & & .9 & & & & & :H & \\
\hline 1.8 & & .9 & & & & & H & 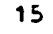 \\
\hline .0 & & & & & & & $\mathrm{H}$ & \\
\hline .5 & & & & & & & H & \\
\hline .2 & & .4 & & & & & H & \\
\hline .8 & & .6 & & & & & & \\
\hline 4.4 & & & & & & & & 1 \\
\hline 3.0 & & 2.0 & -18 & .2 & N1: & & $\mathrm{CH}$ & 15 \\
\hline 2.0 & & .5 & -18 & -18 & C734 & & $\mathrm{CH}$ & 1 \\
\hline 2.9 & & .4 & .6 & -1 & & & $\mathrm{CH}$ & 15 \\
\hline-0.6 & & .7 & -18 & -1 & & & $\mathrm{CH}$ & 1 \\
\hline 2. & & .7 & -18 & .0 & & & $\mathrm{CH}$ & 1 \\
\hline 3. & & .2 & & & & & H & 15 \\
\hline & & .4 & & & & & $\mathrm{H}$ & 1 \\
\hline • & & & & & & & id & \\
\hline 6.4 & & 0.6 & & & & & & 1 \\
\hline 7.0 & & .5 & & & & & & 15 \\
\hline 5. & & & & & & & H & 15 \\
\hline 7. & & & & & & & H & 1 \\
\hline$\bullet \bullet$ & & & & & & & H & 15 \\
\hline & & 1.2 & -18 & & & & & 1 \\
\hline . & & 1. & -18 & -1 & & & H & 15 \\
\hline & & 0.6 & -986 & -1 & N124 & & & 1 \\
\hline & & & -18 & -18 & & & & 15 \\
\hline & & & & & & & & \\
\hline & & & & & & & & \\
\hline
\end{tabular}
ISOS ACC DATE BASE

$-9.86634$ $-11.9 \cos 34$ 
Table 6-(continued) WAME DDG AIN DEG MIN

$P 407 \quad 4 \quad 34.98 \quad 119 \quad 47.00$ P4OB $4134.93 \quad 11944.57$

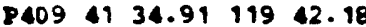
$84104135.03 \quad 11939.96$ $\begin{array}{llllll}P 411 & 41 & 35.99 & 119 & 35.79\end{array}$ $\begin{array}{llllll}P 412 & 41 & 35.53 & 119 & 28.90\end{array}$

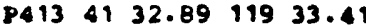
$8414 \quad 1133.75 \quad 11935.17$

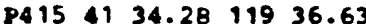
84164130.8111936 .59

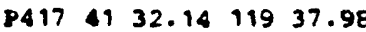
\begin{tabular}{l}
$P 418 \quad 4132.92 \quad 11938.05$ \\
\hline
\end{tabular} $P 41941 \quad 17.83 \quad 119 \quad 28.35$ $\begin{array}{lllll}P 420 & 41 & 14.09 & 119 & 23.91\end{array}$ \begin{tabular}{l}
$842141 \quad 15.6911923 .02$ \\
\hline
\end{tabular} $P 422 \quad 41 \quad 16.53 \quad 119 \quad 20.66$ $P 423 \quad 41 \quad 15.36 \quad 119 \quad 20.50$ $\begin{array}{lllll}P 424 & 41 & 13.68 & 119 & 21.81\end{array}$ $\begin{array}{lllll}P 425 & 41 & 12.31 & 119 & 20.79\end{array}$ P426 $41 \quad 11.48 \quad 119 \quad 21.92$

$827 \quad 41 \quad 19.30 \quad 119 \quad 21.36$ $\begin{array}{lllll}P 428 & 41 & 21.12 & 119 & 24.12\end{array}$ $\begin{array}{lllll}8429 & \$ 1 & 22.20 & 119 & 18.67\end{array}$ $\begin{array}{llllll}P 430 & 41 & 21.33 & 119 & 19.83\end{array}$ $P 4314123.78$
$P 419 \quad 36.76$ \begin{tabular}{l}
$P 4324126.35 \quad 119 \quad 30.83$ \\
\hline
\end{tabular} $P 4334125.59 \quad 11928.76$ $P 434 \quad 4126.99 \quad 11928.89$ $\begin{array}{lllll}P 435 & 41 & 25.82 & 119 & 23.86\end{array}$ $\begin{array}{lllll}P 436 & 41 & 24.46 \quad 119 & 22.75\end{array}$

$8437 \quad 41 \quad 23.82 \quad 119 \quad 20.17$

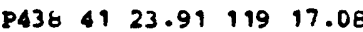

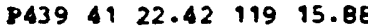
$840 \quad 4124.80 \quad 119 \quad 18.84$
8441

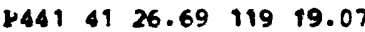
\begin{tabular}{l}
$P 44 \quad \$ 126.18 \quad 119 \quad 21.12$ \\
\hline
\end{tabular}

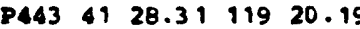
$\begin{array}{llll}P 44 & 49.71 & 119 & 20.46\end{array}$ \begin{tabular}{l}
$P 445129.01 \quad 11922.37$ \\
\hline 446
\end{tabular}

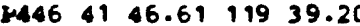

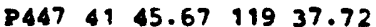
$P 48 \quad 41 \quad 47.30 \quad 119 \quad 35.39$ \begin{tabular}{l}
$P 494144.06 \quad 11937.26$ \\
\hline
\end{tabular} \begin{tabular}{l}
$2450 \quad 4144.63 \quad 11934.62$ \\
\hline 451
\end{tabular}

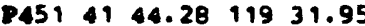

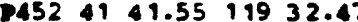
\begin{tabular}{l}
$253 \quad 4139.77 \quad 11932.08$ \\
\hline
\end{tabular}

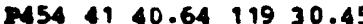
$\begin{array}{lllll}245 & 41 & 40.77 \quad 119 & 27.67\end{array}$ $P 456 \quad 41 \quad 41.86 \quad 119 \quad 27.19$
ELEV OSS GRAV MGAL

5564.7979798 .49 5586.2979795 .18 5661.9979791 .47 5754.7979784 .68 5704.2979788 .18 $6215.0 \quad 979756.40$ 6037.3979756 .49 5885.1979769 .82 5860.2979776 .72 $6478.0 \quad 979724.33$

6358.0979738 .49 6286.0979745 .09 $6979.0 \quad 979679.26$ $6419.0 \quad 979714.55$ 5928.0979749 .76 $5767.0 \quad 979764.17$ 5993.0979745 .84 $5753.0 \quad 979759.92$ $6472.0 \quad 979708.61$ 6327.0979717 .46

6004.0979747 .46 5746.0979759 .72 6609.0979701 .14 $6034.0 \quad 979742.55$ 6832.0979686 .41 6025.0979751 .13 $5655.0 \quad 979769.41$ $5722.0 \quad 979766.23$ $5361.0 \quad 979778.65$ $5751.0 \quad 979752.45$

$6167.0 \quad 979727.81$ 6260.0979723 .33 $5762.0 \quad 979763.47$ $5941.0 \quad 979744.59$ $5798.0 \quad 979754.93$ 5651.0979764 .54 $5770.0 \quad 979759.25$ 5711.0979764 .74 5663.0979769 .24 6724.0979736 .91

$6277.0 \quad 979762.29$ 6312.0979764 .38 6053.0979770 .81 6134.0979768 .57 6187.0979765 .94 6101.0979771 .63 6449.0979745 .33 6235.0979761 .62 5958.0979772 .25 6139.0979758 .00
TREE TERRAIN DOUG

AIK HND COMP NWM

$11.10 .0 f \quad 0.4-179.8$

$\begin{array}{lllllll}9.9 & 0.0 f & 0.3 & -181.8 & -12.6 & 0344\end{array}$

$\begin{array}{lllllll}13.3 & 0.05 & 0.4 & -180.8 & -11.3 & 8124\end{array}$

$15.1 \quad 0.1 f \quad 0.5-182.2 \quad-12.4$ B124

$\begin{array}{llllll}12.1 & 0.08 & 0.4 & -183.4 & -13.1 & 8122\end{array}$

$\begin{array}{llllll}29.3 & 0.1 f & 0.9 & -183.4 & -12.9 & 0634\end{array}$

$\begin{array}{llllll}16.6 & 0.1 f & 0.8 & -190.0 & -19.9 & \mathbf{b} 124\end{array}$

$14.4 \quad 0.18 \quad 0.7-187.1 \quad-17.0 \quad$ N124

$\begin{array}{lllllll}18.1 & 0.05 & 0.6 & -182.6 & -12.6 & \text { N124 }\end{array}$

$29.0 \quad 0.4 f \quad 1.6 \quad-191.8 \quad-22.3 \quad 6634$

$\begin{array}{llllll}29.9 & 0.9 f & 2.2 & -186.3 & -16.7 & 6634\end{array}$

$\begin{array}{lllllll}28.5 & 0.2 f & 1.3 & -186.1 & -16.4 & 6634\end{array}$

$\begin{array}{llllll}50.4 & 2.3 f & 7.6 & -181.5 & -14.7 & \text { V324 }\end{array}$

$\begin{array}{llllll}38.7 & 0.8 f & 3.8 & -178.0 & -12.9 & 6644\end{array}$

$25.4 \quad 2.3 f \quad 3.4-174.9 \quad-9.3 \quad 6644$

$23.4 \quad 0.4 f \quad 1.6 \quad-173.2 \quad-7.8 \quad 6644$

$28.0 \cdot 1.7 f \quad 3.9 \quad-173.9 \quad-9.0 \quad 6644$

$\begin{array}{lllllll}22.1 & 0.1 f & 1.0 & -174.7 & -10.0 & 6644\end{array}$

$\begin{array}{lllll}40.4 & 1.5 f \quad 6.5 & -175.4 & -11.6 & 0634\end{array}$

$\begin{array}{lllllll}36.8 & 2.3 f & 4.7 & -175.8 & -12.0 & 6644\end{array}$

$\begin{array}{llllll}24.8 & 0.4 f & 2.2 & -179.3 & -12.9 & 6644\end{array}$

$\begin{array}{lllllll}10.1 & 0.1 f & 0.8 & -186.6 & -19.1 & 6654\end{array}$

$\begin{array}{llllll}31.0 & 0.6 f & 4.3 & -191.6 & -25.1 & 0324\end{array}$

$\begin{array}{lllllll}19.7 & 0.5 f & 2.2 & -185.5 & -18.8 & 6644\end{array}$

$\begin{array}{lllllll}34.8 & 1.3 f & 5.2 & -194.5 & -25.9 & \text { v324 }\end{array}$

$\begin{array}{lllllll}19.9 & 0.0 f & 0.8 & -186.4 & -17.1 & 6654\end{array}$

$\begin{array}{llllll}4.5 & 0.1 f & 0.5 & -189.3 & -20.3 & 6634\end{array}$

$\begin{array}{lllllll}5.6 & 0.0 f & 0.4 & -190.6 & -21.3 & 6644\end{array}$

$\begin{array}{llllll}-14.2 & 0.8 f & 1.5 & -197.0 & -28.2 & 6644\end{array}$

$\begin{array}{lllllll}-1.7 & 1.7 f & 2.4 & -196.9 & -28.8 & 0644\end{array}$

$\begin{array}{llllll}13.7 & 0.1 f & 1.2 & -196.9 & -29.5 & 6644\end{array}$

$\begin{array}{llllll}17.8 & 0.7 f & 2.4 & -194.8 & -27.8 & 6644\end{array}$

$\begin{array}{lllllll}13.4 & 1.7 f & 3.8 & -180.8 & -14.5 & 6644\end{array}$

$\begin{array}{lllllll}7.8 & 0.2 f & 1.0 & -195.3 & -27.7 & 6644\end{array}$

$\begin{array}{lllllll}1.9 & 0.2 f & 0.8 & -196.6 & -28.3 & 0644\end{array}$

$\begin{array}{llllll}-1.6 & 0.1 f & 0.6 & -195.2 & -26.8 & 6654\end{array}$

$\begin{array}{llllll}1.1 & 0.5 f & 1.0 & -196.2 & -27.3 & 6644\end{array}$

$\begin{array}{llllll}-1.0 & 0.5 f & 1.0 & -196.3 & -27.0 & 0644\end{array}$

$\begin{array}{lllllll}0.0 & 0.65 & 1.1 & -193.5 & -24.2 & 6644\end{array}$

$\begin{array}{lllll}4.0 & 0.1 f \quad 2.6 & -187.2 & -17.7 \quad 6644\end{array}$

$\begin{array}{llllll}25.8 & 0.1 f & 0.9 & -188.9 & -18.9 & 6634\end{array}$

$\begin{array}{lllllll}28.7 & 0.1 f & 1.0 & -187.1 & -17.0 & 6644\end{array}$

$\begin{array}{llllll}15.7 & 0.0 f & 0.6 & -191.6 & -21.5 & 0644\end{array}$

$\begin{array}{lllllll}20.2 & 0.6 f & 1.4 & -189.1 & -18.7 & 6544\end{array}$

$\begin{array}{llllll}23.1 & 0.1 f & 0.9 & -188.6 & -18.0 & 0644\end{array}$

$24.8 \quad 0.1 f \quad 0.7-184.1 \quad-13.5 \quad 6644$

$33.8 \quad 1.4 f \quad 3.0-184.6 \quad-14.10644$

$28.7 \quad 0.0 f \quad 0.7-184.8 \quad-14.1 \quad 6644$

$13.10 .15 \quad 0.6-199.0 \quad-20.10644$

$\begin{array}{llllll}14.3 & 0.35 & 1.0 & -195.6 & -24.7 & 0644\end{array}$
DATE aAse MA-Y NAME

$81685 \mathrm{CH} 15$ $81685 \mathrm{CH} 15$ $81685 \mathrm{CH} 15$ 81685. CH 15 $81885 \mathrm{CH} 15$ $81685 \mathrm{CH} 15$ $81685 \mathrm{CH} 15$ $81685 \mathrm{CH} 15$ $81685 \mathrm{CH} 15$ $81685 \mathrm{CH} 15$

$81685 \mathrm{CH} 15$ $81685 \mathrm{CH} 15$ $81785 \mathrm{CH} 15$ $81785 \mathrm{CH} 15$ $81785 \mathrm{CH} 15$ $81785 \mathrm{CH} 15$ $81785 \mathrm{CH} 15$ $81785 \mathrm{CH} 15$ $81785 \mathrm{CH} 15$ $81785 \mathrm{Ch} 15$

B1785 CH 15 $81785 \mathrm{CH} 15$ $81785 \mathrm{CH} 15$ $81785 \mathrm{CH} 15$ $81785 \mathrm{CH} 15$ $81785 \mathrm{CH} 15$ $81785 \mathrm{CH} 15$ $81785 \mathrm{CH} 15$ $81785 \mathrm{CH} 15$ $81785 \mathrm{CH} 15$

$81785 \mathrm{CH} 15$ $81785 \mathrm{CH} 15$ $81785 \mathrm{CH} 15$ $81785 \mathrm{CH} 15$ $81785 \mathrm{CH} 15$ $81785 \mathrm{CH} 15$ $81785 \mathrm{CH} 15$ $81785 \mathrm{Cr} 15$ $81785 \mathrm{CH} 15$ $81885 \mathrm{CH} 15$

$81885 \mathrm{CH} 15$ $81885 \mathrm{CH} 15$ $81885 \mathrm{CH} 15$ $81885 \mathrm{Ch} 15$ 81885 CH 15 $81885 \mathrm{CH} 15$ $81885 \mathrm{CH} 15$ $81885 \mathrm{CH} 15$ $81885 \mathrm{CH} 15$ $81885 \mathrm{CH} 15$ 
Table 6--(continued)

\begin{tabular}{|c|c|c|c|c|c|c|c|c|c|c|c|c|c|c|c|}
\hline $\begin{array}{l}\text { TATION } \\
\text { WAME }\end{array}$ & & DE & $\begin{array}{l}\text { LONC } \\
\text { DEC }\end{array}$ & $\begin{array}{l}\text { GITUUE } \\
\text { MIN }\end{array}$ & $\begin{array}{l}\text { ELEV } \\
\text { reEs }\end{array}$ & $\begin{array}{l}\text { OBS GRAV } \\
\text { MGAL }\end{array}$ & FREE & $\operatorname{TER}$ & & $\begin{array}{l}\text { BOUG } \\
\text { NWGM }\end{array}$ & $\begin{array}{l}\text { Isos } \\
\text { yOy }\end{array}$ & $\begin{array}{l}\text { ACC } \\
\text { CODE }\end{array}$ & $\begin{array}{r}\text { DATE } \\
M-D-Y\end{array}$ & BAS & ISE \\
\hline$P 457$ & 41 & 42.42 & 119 & 29.03 & 974.0 & 79777.76 & & $0.1 f$ & 0.6 & & -16.1 & 6654 & & & \\
\hline$P 458$ & 41 & 28.26 & 119 & 15.24 & 432.0 & 979715.14 & 9.3 & .41 & 2.2 & -199.4 & -31.5 & C644 & 81885 & $\mathrm{CH}$ & 15 \\
\hline 8459 & 41 & 29.90 & 119 & & 369.0 & 19721.45 & 17.2 & $.4 f$ & 1.7 & -199.8 & -31.3 & 644 & 31885 & $\mathrm{CH}$ & 15 \\
\hline$P 462$ & 40 & 33.18 & 119 & 59.68 & 379.8 & 979794.17 & 12.3 & $1 f$ & 1.0 & -162.0 & -6.8 & N123 & 81985 & $\mathrm{CH}$ & 23 \\
\hline $\begin{array}{l}P 463 \\
P 464\end{array}$ & $\begin{array}{l}40 \\
40\end{array}$ & $\begin{array}{l}3.35 \\
3.09\end{array}$ & $\begin{array}{l}119 \\
119\end{array}$ & & & $\begin{array}{l}979808.16 \\
979810.80\end{array}$ & & & $\begin{array}{l}1.3 \\
1.5\end{array}$ & $\begin{array}{l}-158.5 \\
-156.6\end{array}$ & $\begin{array}{l}-3.5 \\
-1.8\end{array}$ & & $\begin{array}{l}82085 \\
\text { B } 1985\end{array}$ & GERL & \\
\hline$P 465$ & 40 & 35.45 & 119 & 46.47 & 3893.1 & 979815.10 & 40.5 & 0.08 & 0.4 & -174.1 & -19.5 & N123 & 82285 & $\mathrm{CH} 2$ & \\
\hline P466 & 40 & 36.87 & 119 & & 298.5 & 979797.94 & 21.7 & .91 & 2.4 & -167.2 & -10.4 & N124 & 82085 & GERI & L \\
\hline 8467 & 40 & & 119 & & & 979800.90 & -21.0 & . 1f & 1.3 & -166.4 & -10.0 & N124 & 2085 & GLR & \\
\hline P468 & 40 & & 119 & & & 979801.88 & -19.7 & $1 f$ & 1.0 & 5.1 & -9.1 & $N 124$ & 2085 & $\operatorname{GEK}$ & \\
\hline 469 & 40 & 33.90 & 119 & & & 6.78 & 5.9 & 11 & 0.9 & & -4.8 & & 2085 & GEKi & \\
\hline 8470 & 40 & 34.42 & 119 & 55.35 & 541.0 & 979792.93 & -0.2 & $.3 f$ & 0.8 & -155.7 & -0.6 & 6644 & 2085 & GERI & L \\
\hline 8471 & 40 & 35.63 & 119 & 53.76 & 861.0 & 6.47 & 11.6 & $.3 f$ & 0.9 & -154.7 & 0.7 & 6644 & 2085 & GERI & \\
\hline 8472 & 40 & 34.29 & 119 & 52.49 & 0.0 & 4.88 & 12.6 & $.3 f$ & 1.3 & 0.8 & -6.2 & G644 & 2085 & GERI & \\
\hline$P 473$ & 40 & 32.08 & 119 & 51.67 & 4.0 & 0.44 & -5.6 & $.2 f$ & 0.8 & 8.7 & -4.8 & G644 & 2085 & GERI & \\
\hline 474 & 40 & 29.73 & 119 & 51.42 & 2.0 & 9.96 & -21.6 & $.0 f$ & 0.5 & 9.1 & -3.9 & 654 & 82085 & GEKL & \\
\hline 475 & 40 & 28.16 & 119 & 51.63 & 5.0 & 1.79 & -24.3 & .16 & 0.7 & .8 & -8.0 & 534 & 82085 & GERI & \\
\hline 2476 & 40 & 28.74 & 119 & 52 & 3.0 & 8.23 & -5.1 & - If & 0.8 & -1 & -0.9 & G634 & 32085 & GLRI & \\
\hline $\begin{array}{l}P 486 \\
P 487\end{array}$ & $\begin{array}{l}40 \\
40\end{array}$ & & $\begin{array}{l}119 \\
119\end{array}$ & & & & & & $\begin{array}{l}2.6 \\
2.0\end{array}$ & & $\begin{array}{l}-9.8 \\
-4.8\end{array}$ & & & $\begin{array}{l}\text { GERI } \\
\text { GEKI }\end{array}$ & iL \\
\hline P488 & 40 & 43.01 & 119 & 6.05 & 1.0 & 9 & 9.1 & .11 & 1.2 & 1.8 & 13.1 & & & GER & 2 \\
\hline P489 & 40 & 43.99 & 119 & 54.42 & 581.0 & 979719.16 & 8.9 & .91 & 1.9 & -174.5 & -15.4 & 6644 & 2185 & GERI & il \\
\hline 490 & 40 & 41.94 & 119 & 54.09 & 2.0 & 979 & 9.4 & $.4 f$ & 1.8 & -166.8 & -8.8 & 6644 & 2185 & GEKi & i) \\
\hline 8491 & 40 & 41.97 & 119 & 52.20 & 7.0 & 97 & 6.3 & - 16 & 3.5 & 66.3 & -8.5 & 6644 & 2185 & GERI & is) \\
\hline$P 492$ & 40 & 42.38 & 119 & .00 & 2.0 & 97 & -1.9 & $.0 f$ & 1.8 & 3.6 & -5.5 & 6644 & 2185 & GER & \\
\hline 493 & 40 & 40.12 & 119 & 51.44 & 9.0 & 97 & 6.7 & $1.1 f$ & 2.9 & -1 & -8.2 & 6644 & 2185 & GERI & $2 \mathrm{~K}$ \\
\hline 494 & 40 & & 19 & & & 9 & 8. 1 & $5 f$ & 4.4 & & -11.6 & 644 & 185 & $\mathrm{CH}$ & $2-3+2+2$ \\
\hline & 40 & & 119 & & .0 & 9 & .8 & $1 f$ & 1.0 & & 1.5 & & & $\mathrm{CH}$ & 2. $-2+2>3$ \\
\hline 501 & 40 & 80 & 19 & & & 9 & .9 & $7 f$ & 2.0 & -1 & 6.6 & & 185 & $\mathrm{CH}$ & 2. \\
\hline & 40 & & 119 & & & 97 & & & 2.4 & & 9.7 & & & $\mathrm{Cr}$ & \\
\hline & 40 & & 19 & & 9.0 & & & & & & & & & Ct & \\
\hline & 40 & & 19 & & 5.0 & & & & & & .4 & & & Cl & 2 \\
\hline 505 & 40 & & 19 & & 7.0 & 97 & .0 & $8 f$ & .3 & & 0.5 & & 185 & $\mathrm{CH}$ & 2 \\
\hline P506 & 40 & 39.17 & 119 & 56.24 & 6.0 & 97 & 7.8 & $.7 f$ & 12.1 & 2.6 & -9.0 & 324 & 82185 & $\mathrm{CH}$ & 2 \\
\hline 8507 & 40 & 32.40 & 119 & 58.60 & 341.0 & 11.92 & 17.0 & $1.5 t$ & 4.1 & & -8.0 & C644 & 82185 & $\mathrm{CH}$ & \\
\hline$P 509$ & 40 & 37.30 & 119 & 46.85 & 172.0 & 120.19 & -30.8 & $.0 f$ & 0.7 & 6.8 & -11.5 & 654 & 82285 & $\mathrm{CH}$ & 2 \\
\hline P510 & 40 & 35.81 & 119 & 45.47 & 3893.4 & 16.75 & -39.4 & $0.0 f$ & 0.3 & .1 & -18.4 & 124 & 82285 & $\mathrm{CH}$ & 2 \\
\hline P511 & 40 & 38.20 & 119 & 45.38 & 0.0 & 9.97 & -32.5 & $0.0 f$ & 0.8 & 3.4 & -12.8 & 6644 & 82285 & $\mathrm{CH}$ & 23 \\
\hline 512 & 40 & 39.00 & 119 & 46.07 & 7.0 & .07 & 5.4 & $0.0 f$ & 1.0 & .0 & -8.0 & 6634 & 82285 & $\mathrm{Ch}$ & 23 \\
\hline rots & 40 & 30 & 119 & 28 & 6.0 & .72 & .1 & $0.1 f$ & 1.3 & .0 & -5.4 & 6634 & 2285 & $\mathrm{CH}$ & $-1+2+3-1$ \\
\hline 514 & 40 & 41.72 & 119 & 47.83 & 0.0 & & & & & & & & & $\mathrm{CH}$ & \\
\hline & 40 & 43 & 119 & & & 9 & 1.8 & $0.3 f$ & 1.3 & & 4.8 & & 83 & $\mathrm{CH}$ & \\
\hline & 40 & & 119 & & & & -12.9 & & 2.3 & & & & & С甘 & 2 \\
\hline 2517 & 40 & 45.59 & 119 & 50.63 & 4742.0 & 97 & -5.8 & $0.3 f$ & 1.3 & & -8.0 & G & 885. & $\mathrm{CH}$ & 23 \\
\hline 20 & 40 & 26.01 & 119 & & 5135.0 & & 30.5 & $1.1 f$ & 0.9 & -1 & 7.4 & 34 & 82385 & $\mathrm{CH}$ & \\
\hline 29 & 40 & 24.22 & 119 & 59.98 & 5053.0 & 979 & .1 & $0.0 f$ & 0.7 & -1 & 8.3 & & 82385 & $\mathrm{CH}$ & 23 \\
\hline & 40 & & 119 & & 5031.0 & & 30.8 & 0.11 & 0.9 & & 10.3 & 44 & 385 & $\mathrm{CH}$ & \\
\hline 523 & 40 & 22.34 & 119 & 59.68 & 5169.0 & 979750.88 & 34.0 & 0.18 & 0.9 & -1 & 9.1 & 6644 & 82385 & $\mathrm{CH}$ & 23 \\
\hline & 40 & 24 & 199 & & $\begin{array}{l}4010.0 \\
4502.0\end{array}$ & & -8.2 & $0.2 f$ & 0.9 & .4 & 6.5 & 34 & 82385 & $\mathrm{CH}$ & \\
\hline & N & 24. & 10 & & 4502.0 & 979795.81 & 13.2 & $0.4 f$ & 1.8 & -139.9 & 12.1 & 0044 & 82385 & CH & \\
\hline
\end{tabular}


rable 6--(continued)

\begin{tabular}{|c|c|c|c|c|c|c|c|c|c|c|c|c|c|c|c|}
\hline $\begin{array}{l}\text { TATION } \\
\text { Mill }\end{array}$ & $\begin{array}{l}\text { LAT } \\
\text { DLG }\end{array}$ & $\begin{array}{l}\text { IITUDE } \\
\text { HIN }\end{array}$ & $\begin{array}{l}\text { LONG } \\
\text { DEG }\end{array}$ & $\begin{array}{l}\text { GITUUE } \\
\text { MIN }\end{array}$ & $\begin{array}{l}\text { EILV } \\
\text { YEET }\end{array}$ & $\begin{array}{l}\text { OBS GRAV } \\
\text { MGAL }\end{array}$ & $\begin{array}{l}\text { FRXE } \\
\text { AIR }\end{array}$ & $\begin{array}{l}\text { TERR } \\
\text { WWND }\end{array}$ & $\begin{array}{l}\text { AIN } \\
\text { COAP }\end{array}$ & $\begin{array}{l}\text { Doug } \\
\text { nyar }\end{array}$ & $\begin{array}{l}\text { IsOS } \\
\text { ANOA }\end{array}$ & $\begin{array}{l}A C C \\
C O D E\end{array}$ & $\begin{array}{r}\text { DATE } \\
n-D-Y\end{array}$ & & ASE \\
\hline$P 527$ & 40 & 22.77 & 119 & 52.27 & 4272.0 & 979803.75 & 2.6 & $0.1 f$ & 1.1 & -143.2 & 8.3 & 6644 & 82385 & CH & \\
\hline P528 & 40 & 29.71 & 119 & 52.95 & 4192.0 & 979804.59 & -2.5 & 0.08 & 1.5 & -145.3 & 6.3 & 6644 & 62385 & $\mathrm{Ch}$ & 23 \\
\hline P529 & 40 & 20.78 & 119 & 53.88 & 4227.0 & 979801.98 & -0.4 & 0.16 & 2.1 & -143.8 & 7.7 & 6634 & 82385 & $\mathrm{CH}$ & 23 \\
\hline P530 & 40 & 19.23 & 119 & 53.75 & 4205.0 & 979800.15 & -2.0 & $0.0 f$ & 1.0 & -145.7 & 5.5 & C634 & 82385 & $\mathrm{CH}$ & 23 \\
\hline 8531 & 40 & 17.60 & 119 & 52.30 & 4248.0 & 979798.36 & 2.7 & $0.2 f$ & 0.8 & -142.8 & 8.3 & 6644 & 82385 & $\mathrm{CH}$ & 23 \\
\hline P532 & 41 & 33.89 & 119 & 40.10 & 5858.0 & 979775.97 & 17.8 & $0.1 f$ & 0.6 & -183.0 & -13.3 & 6644 & 82485 & $\mathrm{CH}$ & 15 \\
\hline P533 & 41 & 33.04 & 119 & 39.98 & 5852.0 & 979777.70 & 20.2 & $0.2 f$ & 0.8 & -180.1 & -10.4 & 6634 & 82485 & $\mathrm{CH}$ & 15 \\
\hline P534 & 41 & 33.63 & 119 & 43.21 & 5626.0 & 979793.55 & 13.9 & $0.0 \mathrm{f}$ & 0.4 & -179.0 & -9.7 & 6634 & 82485 & $\mathrm{CH}$ & 15 \\
\hline P535 & 41 & 32.37 & 119 & 41.38 & 5709.0 & 979783.05 & 13.1 & $0.0 f$ & 0.5 & -182.6 & -13.1 & 6633 & 82485 & $\mathbf{C H}$ & 15 \\
\hline 8536 & 41 & 31.76 & 119 & 42.64 & 5625.0 & 979787.90 & 11.0 & $0.0 f$ & 0.5 & -181.9 & -12.6 & 6644 & 82465 & $\mathrm{CH}$ & 15 \\
\hline 8537 & 41 & 31.55 & 119 & 40.85 & 686.0 & 4.22 & 13.3 & .06 & 0.6 & -181.4 & -11.9 & 6644 & 2485 & $\mathrm{CH}$ & 15 \\
\hline P536 & 41 & 30.03 & 119 & 40.75 & 5619.0 & 979783.31 & 8.4 & $.0 f$ & 0.8 & -184.0 & 14.5 & 644 & 485 & $\mathrm{CH}$ & 15 \\
\hline P539 & 41 & 28.29 & 119 & 40.98 & 5610.0 & 979780.53 & 7.4 & $1 f$ & 0.7 & -184.7 & 15.4 & 6534 & 2485 & $\mathrm{CH}$ & \\
\hline P540 & 41 & 27.33 & 119 & 41.12 & 5620.0 & 979778.67 & 8.7 & If & 0.7 & -184.1 & 14.8 & 6534 & 485 & $\mathrm{CH}$ & \\
\hline P541 & 41 & 24.74 & 119 & 40.18 & 5652.0 & 979769.59 & 5.7 & .05 & 0.5 & -188.0 & -19.0 & 6534 & 485 & $\mathrm{CH}$ & 15 \\
\hline P542 & 41 & 24.83 & 119 & 41.95 & 5701.5 & 979769.70 & 10.3 & $0.1 f$ & 0.5 & -185.1 & 16.9 & 344 & 585 & $\mathrm{CH}$ & 15 \\
\hline P543 & 41 & 25.31 & 119 & 42.13 & 5947.3 & 979756.62 & 19.6 & $0.2 f$ & 0.9 & -183.8 & -14.9 & 344 & 12585 & $\mathrm{CH}$ & 15 \\
\hline P544 & 41 & 25.76 & 119 & 42.29 & 966.9 & 979756.44 & 20.6 & $0.3 f$ & 1.2 & -183.2 & -14.3 & P344 & 82585 & $\mathrm{CH}$ & 15 \\
\hline$r b 45$ & 41 & 26.27 & 119 & 42.47 & 5639.8 & 979779.49 & 12.1 & $0.2 f$ & 0.6 & -181.1 & -12.1 & P344 & 82585 & $\mathrm{CH}$ & 15 \\
\hline P546 & 41 & & 119 & 42.69 & 593.3 & 2.39 & 9.8 & 0.01 & 0.4 & 32.0 & 2.9 & 344 & 585 & $\mathrm{CH}$ & 15 \\
\hline 47 & 41 & 44 & 119 & 42 & 5585.5 & & 8.8 & $.0 f$ & 0.4 & 2.8 & 3.6 & 44 & 585 & $\mathrm{CH}$ & 15 \\
\hline P548 & 41 & 28.00 & 119 & 43.10 & 5579.6 & 979 & B. 2 & $.0 f$ & 0.4 & -183.2 & -14.1 & P344 & 2565 & $\mathrm{CH}$ & 15 \\
\hline P549 & 41 & 28.58 & 119 & 43.31 & 5575.1 & 5.12 & 8.3 & $.0 f$ & 0.4 & -183.0 & -13.9 & 8344 & 82585 & $\mathrm{CH}$ & 15 \\
\hline P5 50 & 41 & 29.15 & 119 & 43.52 & 5574.5 & $979^{\circ}$ & 8.6 & $.0 f$ & 0.4 & -182.6 & -13.5 & P344 & 82585 & $C_{n}$ & 15 \\
\hline P559 & 41 & 29.72 & 119 & 43.72 & 5570.8 & 979 & 10.3 & $.0 f$ & 0.4 & -180.8 & -11.7 & 8344 & 82585 & $\mathrm{CH}$ & 15 \\
\hline P552 & 41 & 30.30 & 119 & 43.93 & $55 \% 1.1$ & $979^{\circ}$ & 16.1 & $.0 f$ & 0.4 & -181.0 & -11.8 & P344 & 585 & $\mathrm{CH}$ & 15 \\
\hline P553 & 41 & 88 & 119 & 44.12 & 5578.3 & 979 & 9.3 & .01 & 0.4 & 2.1 & -12.9 & 44 & 85 & $\mathrm{CH}$ & 15 \\
\hline & 41 & 31 & 119 & 44.86 & $570 y .0$ & & 13.1 & $.2 f$ & 0.6 & 2.6 & 1.6 & 44 & 85 & $\mathrm{CH}$ & 15 \\
\hline P555 & 41 & 31. & 119 & 43.60 & 5624.0 & & 11.6 & $.1 f$ & 0.5 & 1.2 & 2.0 & 6644 & 585 & $\mathrm{Ch}_{\mathrm{H}}$ & 15 \\
\hline 1556 & 41 & 31.46 & 119 & 44.32 & 5624.5 & & 11.1 & $0.1 f$ & 0.5 & -181.7 & -12.6 & 344 & & & 15 \\
\hline & 41 & & 119 & 44. & 00.3 & & 1.1 & $.2 f$ & .6 & & & & & & 15 \\
\hline 8558 & 41 & 32.70 & 119 & 44.72 & 5663.2 & 979788.12 & 13.4 & $0.2 f$ & 0.6 & -180.7 & -11.6 & $P 344$ & 82585 & $\mathrm{CH}$ & 15 \\
\hline P559 & 41 & 33.25 & 119 & 44.91 & 5598.3 & 979793.59 & 11.9 & $0.1 f$ & 0.4 & -180.1 & -10.9 & P344 & 82585 & $\mathrm{CH}$ & 15 \\
\hline P560 & 41 & 33.84 & 119 & 45.10 & 5562.3 & 979796.65 & 10.7 & $0.0 f$ & 0.3 & -180.1 & -11.0 & P344 & 82585 & $\mathrm{CH}$ & 15 \\
\hline P561 & 41 & 34.38 & 119 & 45.26 & 5569.5 & 979797.00 & 10.9 & $0.0 f$ & 0.3 & -180.2 & -11.1 & P344 & 82585 & $\mathrm{CH}$ & 15 \\
\hline P562 & 41 & 53.70 & 119 & 44.31 & 5568.0 & 979796.65 & 11.5 & $0.0 f$ & 0.4 & -179.5 & -10.3 & 6634 & 82585 & $\mathrm{CH}$ & 15 \\
\hline P563 & 41 & 34.87 & 119 & 45.41 & 5589.1 & 979796.29 & 19.3 & $0.0 f$ & 0.3 & -180.4 & -11.4 & P344 & 82585 & $\mathrm{CH}$ & 15 \\
\hline 2564 & $4 !$ & 35.43 & 119 & 45.58 & 5577.6 & 979796.34 & 9.5 & $0.0 f$ & 0.3 & -181.9 & -12.8 & P344 & 82585 & $\mathrm{CH}$ & 15 \\
\hline P565 & 41 & 35.97 & 119 & 45.71 & 5574.3 & 979798.02 & 10.0 & $0.0 f$ & 0.3 & -181.2 & -12.2 & P344 & 82585 & $\mathrm{CH}$ & 15 \\
\hline P566 & 41 & 36.53 & 119 & 45.90 & 5567.3 & 979798.99 & 9.5 & 0.08 & 0.3 & -181.5 & & & 0200 & $\mathrm{CH}$ & 15 \\
\hline P567 & 41 & 37.11 & 119 & 46.08 & 559.2 & & 9.5 & $.0 E$ & 0.4 & 1.2 & -12.2 & P344 & & $\mathrm{CH}$ & 15 \\
\hline 568 & 41 & 37.66 & 119 & 46.25 & 5561.8 & 97 & 7.2 & $0.0 f$ & 0.4 & -183.6 & -14.6 & 8344 & 585 & $\mathrm{CH}$ & 15 \\
\hline $\begin{array}{l}\text { P569 } \\
\text { P570 }\end{array}$ & $\begin{array}{l}41 \\
41\end{array}$ & $\begin{array}{l}38.38 \\
38.93\end{array}$ & $\begin{array}{l}119 \\
119\end{array}$ & $\begin{array}{l}46.49 \\
46.71\end{array}$ & $\begin{array}{l}5557.5 \\
5556.5\end{array}$ & $\begin{array}{l}979799.12 \\
979801.92\end{array}$ & $\begin{array}{l}5.9 \\
7.8\end{array}$ & $\begin{array}{l}0.0 f \\
0.1 f\end{array}$ & $\begin{array}{l}0.5 \\
0.6\end{array}$ & $\begin{array}{l}-184.6 \\
-182.6\end{array}$ & $\begin{array}{l}-15.6 \\
-13.6\end{array}$ & $\begin{array}{l}\text { P344 } \\
\text { P344 }\end{array}$ & $\begin{array}{l}82585 \\
82585\end{array}$ & $\begin{array}{l}\mathrm{CH} \\
\mathrm{CH}\end{array}$ & 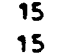 \\
\hline$P 571$ & 41 & 39.36 & 119 & 47.03 & 5549.6 & & 10.0 & $0.0 f$ & 0.5 & & -11.4 & $P 344$ & 85 & $\mathrm{CH}$ & 15 \\
\hline & 41 & & 119 & & 5526.7 & & 6.7 & $0.0 f$ & 0.5 & & -14.0 & $P 344$ & 85 & $\mathrm{CH}$ & 15 \\
\hline 2573 & 41 & 40.51 & 119 & 47.92 & 5524.1 & 979802.45 & 2.9 & $0.0 f$ & 0.4 & -186.5 & -17.8 & P344 & 82585 & $\mathrm{EH}$ & 15 \\
\hline 8574 & 41 & 41.16 & 119 & 48.40 & 5513.3 & 979799.41 & -2.1 & $0.0 f$ & 0.4 & -191.2 & -22.6 & 2344 & 82585 & $\mathrm{CH}$ & 15 \\
\hline $\begin{array}{l}P 575 \\
P 576\end{array}$ & $\begin{array}{l}41 \\
41\end{array}$ & $\begin{array}{l}41.81 \\
42.36\end{array}$ & $\begin{array}{l}119 \\
119\end{array}$ & $\begin{array}{l}48.70 \\
48.94\end{array}$ & $\begin{array}{l}5526.1 \\
5523.5\end{array}$ & $\begin{array}{l}979797.85 \\
979798.97\end{array}$ & $\begin{array}{l}-3.4 \\
-3.4\end{array}$ & $\begin{array}{l}0.0 f \\
0.0 f\end{array}$ & $\begin{array}{l}0.4 \\
0.4\end{array}$ & $\begin{array}{l}-193.0 \\
-192.8\end{array}$ & $\begin{array}{l}-24.4 \\
-24.3\end{array}$ & $\begin{array}{l}\text { P344 } \\
\text { P344 }\end{array}$ & $\begin{array}{l}82585 \\
82585\end{array}$ & $\begin{array}{l}\mathrm{CH} \\
\mathrm{CH}\end{array}$ & $\begin{array}{l}15 \\
15\end{array}$ \\
\hline
\end{tabular}


rable 6--(continued)

STATION LATITUUE LONGITUDE MHAE DLG MIN DEG MIN

$\begin{array}{lllll}P 577 \quad 41 \quad 42.91 & 119 \quad 49.16\end{array}$ $\begin{array}{llllll}P 578 & 41 & 43.44 & 119 & 49.36\end{array}$

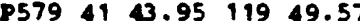

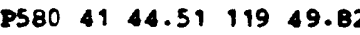
$P 5814145.1111950 .07$ P582 $4145.67 \quad 11950.28$ $P 583 \quad 4146.28 \quad 11950.38$

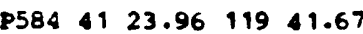

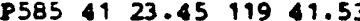

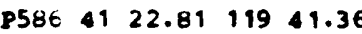

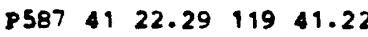
PSBE $4121.75 \quad 119 \quad 41.07$

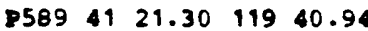

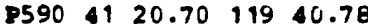
$\begin{array}{lllll}P 591 \quad 4120.18 \quad 119 & 40.63\end{array}$ PS92 4119.6111940 .47 P593 $41 \quad 19.03 \quad 11940.3$ $\begin{array}{llllll}\text { P594 } 41 & 18.43 \quad 119 & 40.15\end{array}$ $\begin{array}{llllll}P 595 \quad 41 & 17.82 \quad 119 & 40.23\end{array}$ P596 $41 \quad 17.26 \quad 119 \quad 46.48$

$P 597 \quad 41 \quad 16.73 \quad 119 \quad 37.72$ $P 598 \quad 41 \quad 17.32 \quad 11938.91$ $\begin{array}{lllll}P 599 & 41 & 16.73 & 119 & 40.72\end{array}$ $\begin{array}{llllll}P 600 & 16.20 & 119 & 40.57\end{array}$ P601 $41 \quad 15.65 \quad 11941.22$ P602 $41 \quad 15.08 \quad 11941.48$

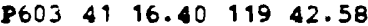
\begin{tabular}{l}
$P 04 \quad 41 \quad 15.17 \quad 119 \quad 43.02$ \\
\hline 600549
\end{tabular}

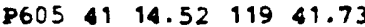
PtDó $41 \quad 13.98 \quad 11941.98$

$P 607 \quad 4 \quad 13.42 \quad 119 \quad 41.98$

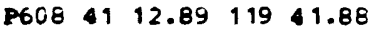
P609 $41 \quad 12.34119 \quad 41.78$ P610 $4111.76119 \$ 1.66$

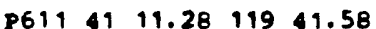

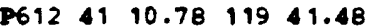
P613 $41 \quad 10.05 \quad 11941.35$

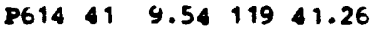
P615 $419.06 \quad 11941.18$ $P 16 \quad 41 \quad 8.4911941 .07$

$\begin{array}{lllll}P 617 & 41 & 7.90 & 119 & 40.96\end{array}$ PE18 $41 \quad 7.23 \quad 119 \quad 40.84$ $\begin{array}{lllll}P 619 & 41 \quad 6.69 \quad 119 \quad 40.74\end{array}$ $\begin{array}{lllll}P 620 & 41 & 47.13 \quad 119 & 49.38\end{array}$ P621 $4146.83 \quad 11950.49$ $\% 2241 \quad 47.35 \quad 11950.57$ P623 4148.0111949 .68

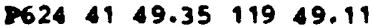
$P 25 \quad 41 \quad 49.05 \quad 119 \quad 49.81$

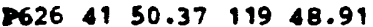

DLEV OSS GRAV
FEET NGH

5521.9979801 .13 5520.9979803 .63 5525.0979804 .78 5518.3979805 .99 5524.3979810 .22 5523.3979813 .06 5526.2979815 .38 5765.1979764 .62 5721.2979767 .68 5756.2979765 .39

5737.3979765 .26 $5805.8 \quad 979759.61$ 5840.2979757 .99 $6041.8 \quad 979744.09$ 5855.1979755 .79 6030.7979741 .73 6274.9979724 .93 $5915.8 \quad 979750.70$ 5723.4979759 .71 5659.2979757 .83

$5922.0 \quad 979748.63$ $5787.0 \quad 979752.18$ 5600.2979761 .51 5607.4979762 .63 $5633.8 \quad 979763.66$ 5714.6979757 .35 $5635.0 \quad 979763.67$ $5825.0 \quad 979753.35$ 5821.2979751 .20 5914.2979743 .04

$6015.6 \quad 979736.19$ 6075.7979732 .22 6314.1979715 .26 6386.9979706 .96 6360.7979709 .83 6223.3979716 .20 6046.9979726 .96 6153.7979710 .72 6102.1979720 .44 6346.3979702 .38

6347.6979700 .34 6257.2979704 .01 6001.4979720 .09 5578.0979812 .90 $5565.0 \quad 979812.01$ 5721.3979803 .30 5689.0979809 .13 5682.0979811 .41 $5682.0 \quad 979810.38$ 5683.0979816 .43

\begin{tabular}{|c|c|c|c|c|c|c|c|c|}
\hline & & & $\begin{array}{l}\text { BOUG } \\
\text { WOM }\end{array}$ & $\begin{array}{l}\text { Isos } \\
\text { MNay }\end{array}$ & $\begin{array}{l}\text { ACC } \\
\text { CODE }\end{array}$ & $\begin{array}{r}\text { DhTE } \\
M-D-Y\end{array}$ & & ASE \\
\hline $\begin{array}{r}-0.6 \\
0.2 \\
-0.1\end{array}$ & $\begin{array}{l}0.0 f \\
0.0 f \\
0.0 f \\
0.0 f\end{array}$ & $\begin{array}{l}0.4 \\
0.4 \\
0.4 \\
0.4\end{array}$ & & $\begin{array}{l}-23.1 \\
-21.0 \\
-21.0 \\
-21.0\end{array}$ & & & & $\begin{array}{l}15 \\
15 \\
15 \\
15\end{array}$ \\
\hline $\begin{array}{l}3.8 \\
5.8\end{array}$ & & & & & & & :H & \\
\hline 7.4 & $0.0 f$ & 0.7 & 1.9 & $3 . B$ & P344 & 82585 & CH & 15 \\
\hline 2.5 & & 0.8 & & 5.9 & P334 & & $\mathrm{CH}$ & 15 \\
\hline 2.8 & $1 f$ & .6 & .8 & & & & $\mathrm{CH}$ & 15 \\
\hline 1.2 & $5 f$ & .0 & & & & & $\mathrm{CH}$ & 15 \\
\hline 1.1 & $1 f$ & .6 & & & & & $\mathrm{CH}$ & 15 \\
\hline 6.6 & $2 f$ & 1.7 & -18 & & P3 & & $\mathrm{CH}$ & 15 \\
\hline 6.9 & $1 f$ & .6 & -1 & 4.6 & $P$ & & $\mathrm{CH}$ & 15 \\
\hline 2.9 & $3 f$ & 0 & & & & & $\mathrm{CH}$ & 15 \\
\hline 1.8 & if & .7 & & & & & $\mathrm{CH}$ & 15 \\
\hline 1.8 & 16 & .8 & & & & & $\mathrm{CH}$ & 15 \\
\hline • & & & & & & & $\mathrm{CH}$ & 15 \\
\hline 1.0 & & & & & & & $\mathrm{CH}$ & 15 \\
\hline 2.9 & & & & & $P$ & & $\mathrm{CH}$ & 15 \\
\hline 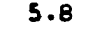 & & & & & & & $\mathrm{CH}$ & 15 \\
\hline . & & .7 & & & & & $\mathrm{CH}$ & 15 \\
\hline & & & & & & & $\mathrm{CH}$ & 15 \\
\hline 4. & $O E$ & 0.6 & & & & & $\mathrm{CH}$ & 1 \\
\hline 7. & & .6 & & & & & $\mathrm{CH}$ & 15 \\
\hline 1. & & .6 & & & & & $\mathrm{CH}$ & 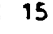 \\
\hline 3.8 & & 0.6 & & & & & $\mathrm{CH}$ & 1 \\
\hline 10.7 & & 0.6 & & & & & $\mathrm{CH}$ & 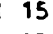 \\
\hline 0. & & 0.6 & & & & & $\mathrm{CH}$ & \\
\hline 3. & & & & & & & $\mathrm{Cr}$ & \\
\hline 9. & & & & & & & $\mathrm{CH}$ & 15 \\
\hline . & & . 9 & & & & & $\mathrm{Cr}$ & 115 \\
\hline 5.5 & $3 f$ & .1 & & -14.6 & & & $\mathrm{CH}$ & 15 \\
\hline 2 . & $3 t$ & .4 & & 6.4 & & & $\mathrm{CH}$ & 15 \\
\hline 3. & $4 f$ & .6 & & 7.5 & 344 & & $\mathrm{CH}$ & 15 \\
\hline 2. & & .4 & & & & & $\mathrm{CH}$ & 15 \\
\hline b & & & & & & & $\mathrm{CH}$ & 15 \\
\hline 22.8 & $2 f$ & .1 & & & & & $\mathrm{CH}$ & 15 \\
\hline 4. & & & & & & & $\mathrm{CH}$ & 15 \\
\hline & & & & & & & $\mathrm{CH}$ & 15 \\
\hline & & & & & & & & \\
\hline 7. & & & & & & & C & 15 \\
\hline 3. & & & & & & & $\mathrm{CH}$ & 15 \\
\hline 16. & & & & & & & CH & 15 \\
\hline 8. & & & & & & & $\mathrm{CH}$ & 15 \\
\hline & - If & .9 & & -15 & P354 & 85 & $\mathrm{CH}$ & 15 \\
\hline 12 & $1.6 f$ & & & & & 85 & $\mathrm{CH}$ & 15 \\
\hline & & & & & & 85 & $\mathrm{CH}$ & 15 \\
\hline & & .5 & & & 0634 & 82785 & $\mathrm{CH}$ & 15 \\
\hline & $\begin{array}{l}0.01 \\
0.11\end{array}$ & .7 & & $\begin{array}{r}-13.7 \\
-9.5\end{array}$ & & $\begin{array}{l}82785 \\
82785\end{array}$ & $\begin{array}{l}\mathrm{CH} \\
\mathrm{CH}\end{array}$ & \\
\hline
\end{tabular}


Table 6--(continued)

STATION IATITUDE LONGITUDE WUIE UDG MIN DEG MIN

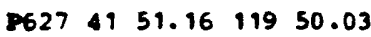
$P 28$
$4152.06 \quad 11951.15$ $8294154.68 \quad 11954.05$
8630 $8630 \triangle 154.3311955 .10$ P631 4153.7911954 .86 $P 6324153.2511954 .61$ $\begin{array}{lllll}2633 & 41 & 52.73 & 119 & 54.37\end{array}$ \begin{tabular}{l}
$P 634$ \\
\hline $1152.23 \quad 11954.17$
\end{tabular} $\begin{array}{lllll}P 635 & 4151.75 \quad 11953.79\end{array}$ $P 6364151.2911953 .43$

$P 637 \quad 4150.75 \quad 119 \quad 52.99$ $\begin{array}{llllll}8638 & 4150.28 \quad 119 & 52.65\end{array}$ \begin{tabular}{l}
$P 6394149.84 \quad 11952.31$ \\
\hline
\end{tabular} \begin{tabular}{l}
$P 40 \quad 4149.44 \quad 11951.85$ \\
\hline
\end{tabular} $\begin{array}{llll}P 64141 & 49.00 \quad 119 & 51.38\end{array}$

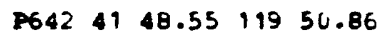
$\begin{array}{llll}P 643 \quad 4154.86 \quad 119 & 55.35\end{array}$ P644 $4155.40 \quad 11955.61$ $\begin{array}{lllll}P 645 & 41 & 55.93 \quad 119 & 55.85\end{array}$ $\begin{array}{llll}P 646 \quad 41 & 54.45 \quad 119 & 56.91\end{array}$

$\begin{array}{lllll}P 647 \quad 41 & 53.20 \quad 119 & 59.04\end{array}$ $\begin{array}{llllll}\text { PE19 } & 41 & 55.18 & 118 & 13.36\end{array}$ $\begin{array}{lllll}P 820 & 41 & 54.26 & 118 & 14.69\end{array}$ $\begin{array}{lllll}P 821 & 4153.19 & 118 & 15.71\end{array}$ $\begin{array}{lllll}\text { P822 } & 41 & 53.46 & 118 & 17.42\end{array}$ $\begin{array}{lllll}\text { P823 } & 41 & 51.80 & 118 & 16.44\end{array}$ $\begin{array}{lllll}\text { P82 } & 41 & 50.19 & 118 & 16.20\end{array}$ $\begin{array}{lllll}P 646 & 41 & 31.42 & 119 & 4.39\end{array}$ $\begin{array}{llllll}\text { PB47 } & 41 & 29.89 & 119 & 4.49\end{array}$ $\begin{array}{llllll}\text { P848 } & 41 & 28.02 & 119 & 6.82\end{array}$

$\begin{array}{lllll}P 849 & 41 & 26.79 & 119 & 7.32\end{array}$ $\begin{array}{lllll}P 850 & 41 & 25.12 & 119 & 9.17\end{array}$ $\begin{array}{lllll}P 851 & 41 & 25.30 & 119 & 3.10\end{array}$ $\begin{array}{llllll}\text { P852 } & 41 & 22.83 & 119 & 0.95\end{array}$ $\begin{array}{lllll}P 853 & 41 & 23.20 & 118 & 59.60\end{array}$ $\begin{array}{lllll}P 854 & 41 & 24.23 & 118 & 56.37\end{array}$ $\begin{array}{lllll}P 855 & 41 & 24.60 & 118 & 59.64\end{array}$ $P 856 \quad 4122.00 \quad 118 \quad 51.91$

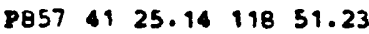
$\begin{array}{lllll}P 858 & 41 \quad 26.90 \quad 118 & 48.66\end{array}$

\section{DEEV OBS GRAV FEET MGAL}

$5684.0 \quad 979818.38$ 6443.0979770 .91 5743.0979815 .01 5718.9979817 .13 5795.3979813 .71 $5889.0 \quad 979807.28$ 5956.6979799 .96 6080.2979790 .00 6141.8979786 .64 6260.7979778 .71

6406.0979771 .10 $6509.0 \quad 979764.17$ 6605.9979753 .54 6652.9979749 .20 6280.2979773 .00 6094.6979783 .10 $5708.8 \quad 979817.77$ $5730.8 \quad 979817.34$ $5697.6 \quad 979822.07$ $5706.0 \quad 979820.72$

$5404.0 \quad 979838.92$ $5476.0 \quad 979845.91$ $4802.0 \quad 979876.98$ $4468.0 \quad 979883.86$ $4533.0 \quad 979878.88$ $4432.0 \quad 979885.32$ $4345.0 \quad 979890.95$ 5865.0979765 .01 5963.7979756 .08 $4988.0 \quad 979822.85$

$4862.0 \quad 979830.23$ 4728.0979840 .44 5830.0979769 .18 6516.0979735 .96 6984.0979701 .70 7280.0979679 .76 8012.0979626 .61 4061.5979884 .29 $\$ 082.9979882 .33$ 4094.4979885 .50

\begin{tabular}{|c|c|c|c|c|c|c|c|}
\hline $\begin{array}{l}\text { FREE. } \\
\text { AIR }\end{array}$ & HWNO & IN & $\begin{array}{l}\text { DOUG } \\
\text { MNan }\end{array}$ & $\begin{array}{l}\text { IsOS } \\
\text { ANar }\end{array}$ & $\begin{array}{l}\text { ACC } \\
\text { CODE }\end{array}$ & $\begin{array}{r}\text { DATE } \\
M-\Sigma-Y\end{array}$ & $\begin{array}{l}\text { mas } \\
\operatorname{may}\end{array}$ \\
\hline 17.9 & $0 E$ & . 9 & 76.5 & -8.7 & 644 & 82785 & $\mathrm{CH}$ \\
\hline 40.5 & .41 & .2 & 177.7 & -10.4 & 6644 & 82785 & $\mathrm{CH} 9$ \\
\hline 14.8 & .08 & .8 & 181.8 & -14.9 & 634 & 82785 & $\mathrm{CH}$ \\
\hline 15.2 & of & 6 & 7 & -14.0 & 143 & 2785 & CH 1 \\
\hline 19.8 & & 7 & & 11.9 & & 2785 & CH 1 \\
\hline 23 & & & & & & & 41 \\
\hline 2 & & 8 & & & & 785 & $\mathrm{CH}$ \\
\hline 25.2 & If & .0 & 82.7 & 5.9 & 44 & 2785 & $\mathrm{CH}$ \\
\hline 8.3 & $1 f$ & .0 & 31.6 & -14.6 & 8344 & 2785 & $\mathrm{CH}$ \\
\hline 32.3 & $.1 f$ & 1.2 & 1.6 & .14 .6 & 8344 & 12785 & $\mathrm{CH} 1$ \\
\hline & & .5 & -179.3 & -12.2 & & 2785 & $\mathrm{CH}$ \\
\hline 42.6 & . $1 f$ & .7 & -179.2 & -12.1 & 54 & 2785 & $\mathrm{CH}$ \\
\hline 1.7 & & .2 & -182.9 & -15.8 & & 2785 & $\mathrm{CH}$ \\
\hline 42.4 & & .0 & 3.1 & -15.8 & & 2785 & $\mathrm{CH}$ \\
\hline 31.8 & & .0 & 1.9 & 14.4 & & 2785 & $\mathrm{CH}$ \\
\hline 25.2 & & 4 & 2.8 & -15.1 & 344 & 2785 & $\mathrm{CH}$ \\
\hline 14.1 & & .6 & 1.5 & -14.9 & & 2785 & $\mathrm{CH}$ \\
\hline 14.9 & & 6 & 1.4 & & & 2785 & $\mathrm{Cri}$ \\
\hline 15.8 & & 6 & 9.4 & & & & $\mathrm{CH}$ \\
\hline 17.4 & & & & & & & $\mathrm{Cl}$ \\
\hline & & & & & & & C \\
\hline 19.5 & .ef & .5 & 5.8 & -0.4 & G734 & 1586 & $M C D$ \\
\hline 11.0 & . $2 f$ & 1.9 & $-174 \cdot 3$ & -8.8 & 6734 & 1586 & $M C D$ \\
\hline 3.9 & $.0 f$ & 1.7 & -185.9 & 20.6 & G754 & 1586 & MCD \\
\hline 3 & 06 & .9 & -187.2 & -22.0 & 6734 & 586 & MCD \\
\hline 33.8 & $.0 f$ & .5 & -184.7 & -19.7 & G734 & 1586 & $\mathrm{MCD}$ \\
\hline 3.9 & & .5 & 1.9 & 17.2 & & & MCD \\
\hline & & .0 & & & & & $D E$ \\
\hline 13.8 & of & . 3 & 9.8 & 3.2 & & 786 & DE \\
\hline- & & & & & & & \\
\hline & & 1.5 & & & & & $D E$ \\
\hline-10.9 & & 1.6 & -1 & & & & $D E$ \\
\hline & $7 f$ & .9 & -176.3 & .8 & G) 44 & 81786 & DENI \\
\hline 56. & $0.4 f$ & 3.0 & -164.6 & -2.0 & G744 & 81786 & DENI \\
\hline 65. & $0.5 f$ & 4.9 & -169 & -7.3 & G7 & 81786 & DENI \\
\hline & & 9.6 & -170 & -8.2 & G7. & 81786 & $D E$ \\
\hline 84. & $2.1 f$ & 13.8 & -176.3 & -13.6 & 6744 & 81786 & DENI I \\
\hline 25.0 & $0.0 f$ & 1.4 & -163.4 & -2.9 & N224 & 81786 & DENI \\
\hline & 0.05 & 2.4 & -167.8 & & N224 & 81786 & \\
\hline & $0\}$ & & 967 & & 8124 & 81786 & \\
\hline
\end{tabular}


Table 7-Gravity dete collected in Oregon.

\begin{tabular}{|c|c|c|c|c|c|c|c|c|c|c|c|c|c|c|}
\hline $\begin{array}{l}\text { EATION } \\
\text { WhHE }\end{array}$ & $\begin{array}{l}\text { LAT } \\
\text { DEC }\end{array}$ & $\begin{array}{l}\text { ITUDE } \\
\text { MIN }\end{array}$ & $\begin{array}{l}\text { LONG } \\
\text { DDC }\end{array}$ & $\begin{array}{l}\text { GITUDE } \\
\text { MIN }\end{array}$ & $\begin{array}{l}\text { ELEV } \\
\text { FEET }\end{array}$ & $\begin{array}{l}\text { OBS GRAV } \\
\text { MGAL }\end{array}$ & $\begin{array}{l}\text { FREE } \\
\text { AIR }\end{array}$ & $\begin{array}{l}\text { TERS } \\
\text { WAND }\end{array}$ & & $\begin{array}{l}\text { Doug } \\
\text { wax }\end{array}$ & $\begin{array}{l}\text { Isos } \\
\text { NWOH }\end{array}$ & $\begin{array}{l}A C C \\
\infty D L\end{array}$ & $\begin{array}{r}\text { DATE } \\
M-D-Y\end{array}$ & $\begin{array}{l}\text { BASE } \\
\text { WAME }\end{array}$ \\
\hline$P 230$ & 42 & 23 & 118 & 37.51 & 196.8 & 99936.75 & -18.6 & 0.41 & 4.9 & -158.1 & 6.2 & 8124 & 101484 & DENIO \\
\hline 2231 & 42 & 92 & 118 & 36.64 & 248.2 & 79939.81 & -13.2 & $0.2 f$ & 4.3 & & 8.9 & b124 & 101484 & DENIO \\
\hline P232 & 42 & .38 & 118 & 37.59 & 562.0 & 979867.47 & 38.2 & $.0 f$ & 7.9 & -152.0 & 11.0 & 6634 & 101484 & DENIO \\
\hline $\begin{array}{l}\text { P233 } \\
P 234\end{array}$ & $\begin{array}{l}42 \\
42\end{array}$ & $\begin{array}{l}7.96 \\
9.26\end{array}$ & $\begin{array}{l}118 \\
118\end{array}$ & & $\begin{array}{r}4189.0 \\
4191.4\end{array}$ & $\begin{array}{l}979944.62 \\
979944.42\end{array}$ & $\begin{array}{l}-21.5 \\
-23.5\end{array}$ & $\begin{array}{l}0.2 f \\
0.28\end{array}$ & $\begin{array}{l}3.1 \\
1.9\end{array}$ & $\begin{array}{l}-162.6 \\
-165.8\end{array}$ & $\begin{array}{r}0.7 \\
-2.9\end{array}$ & $\begin{array}{l}\text { C634 } \\
\text { B123 }\end{array}$ & $\begin{array}{l}101484 \\
101684\end{array}$ & $\begin{array}{l}\text { DENIO } \\
\text { DENIIO }\end{array}$ \\
\hline $\begin{array}{l}P 235 \\
P 236\end{array}$ & $\begin{array}{l}42 \\
42\end{array}$ & $\begin{array}{l}10.77 \\
10.79\end{array}$ & $\begin{array}{l}118 \\
118\end{array}$ & & $\begin{array}{l}1162.6 \\
\$ 136.0\end{array}$ & $\begin{array}{l}979947.68 \\
979943.63\end{array}$ & $\begin{array}{l}-25.2 \\
-31.7\end{array}$ & $\begin{array}{l}0.1 f \\
0.0 f\end{array}$ & $\begin{array}{l}1.3 \\
0.9\end{array}$ & $\begin{array}{l}-167.1 \\
-173.2\end{array}$ & $\begin{array}{r}-4.4 \\
-10.6\end{array}$ & $\begin{array}{l}V 124 \\
6534\end{array}$ & $\begin{array}{l}101484 \\
101484\end{array}$ & $\begin{array}{l}\text { DENIO } \\
\text { DENIO }\end{array}$ \\
\hline 8237 & 42 & 11.94 & 118 & & 161.0 & 979949.77 & -25.0 & 0.01 & 1.0 & -167.2 & -4.7 & 6633 & 101484 & DENIO \\
\hline P238 & 42 & 10.42 & 118 & 38.97 & 4500.0 & 979937.81 & -2.8 & $.6 f$ & 3.0 & -154.7 & 8.1 & 6644 & 101484 & DENIO \\
\hline 8239 & 42 & 11.40 & 118 & & 4240.0 & 150.07 & & .38 & 1.7 & & 1.9 & 634 & 484 & DENIO \\
\hline 240 & 42 & & 198 & & 5.0 & 1.08 & 1.1 & $.3 f$ & 1.7 & & 12.0 & 734 & 484 & ENIO \\
\hline 8241 & 42 & 10.42 & 118 & 41.08 & 5461.0 & & 35.9 & .78 & 2.5 & & 12.6 & 644 & 484 & DENIO \\
\hline$P 242$ & 42 & 6.74 & 118 & 35.16 & 4194.0 & 61 & -23.2 & - $1 f$ & 2.8 & -16 & -1.3 & 6634 & 1484 & DENIO \\
\hline 8243 & 42 & 65 & 118 & 34.32 & 7.0 & & -27.5 & $2 f$ & 2.0 & & -5.6 & N224 & 484 & DNilo \\
\hline 8244 & 42 & 0.38 & 118 & 48.35 & 4423.2 & & -26.4 & of & 1.1 & & -12.4 & 123 & 384 & ENIO \\
\hline$P 245$ & 42 & 1.04 & 118 & 48.26 & 4470.9 & & -23.5 & & 1.3 & & -11.0 & 124 & 84 & ENIO \\
\hline 246 & 42 & 68 & 118 & .12 & 10.0 & .53 & -21.8 & 0.01 & 1.6 & -174.4 & -9.5 & N124 & 101584 & DNIO \\
\hline$\$ 247$ & 42 & 55 & 118 & 47.72 & 4570.9 & 979904.82 & -17.3. & $0.1 f$ & 1.8 & -172.8 & -8.0 & N124 & 101584 & DENIO \\
\hline P248 & 42 & 4.05 & 118 & 45.74 & 5173.0 & 979871.88 & 4.1 & $0.3 f$ & 2.3 & -171.5 & -7.3 & 6634 & 101584 & DENIO \\
\hline$\$ 249$ & 42 & 26 & 118 & 47 & 2.2 & & & & 1.7 & & -7.8 & 124 & 84 & ENIO \\
\hline & 42 & 89 & 118 & & & & & & 2.4 & & -7.2 & & & 10 \\
\hline & 42 & 21 & 118 & & .7 & & & & 1.3 & & -9.4 & 124 & 84 & iIO \\
\hline 52 & 42 & 10 & 118 & & 1.6 & & 3.0 & If & 2.0 & & -3.5 & N124 & 584 & ENIO \\
\hline 253 & 42 & .80 & 118 & 47 & 3.0 & & 1.4 & of & 1.4 & & -6.9 & D333 & 986 & Elí \\
\hline 254 & 42 & 11.45 & 118 & & .9 & & .1 & & .4 & & -2.7 & N124 & 84 & ENIO \\
\hline 55 & 42 & 12.49 & 118 & 46 & .0 & & .6 & $2 f$ & 6.7 & & 2.0 & 6634 & 84 & ENIO \\
\hline 256 & 42 & 41 & 118 & & .2 & & & & 2.4 & & -7.1 & N124 & 584 & ENio \\
\hline & 42 & .02 & 118 & & .0 & & -11.1 & 15 & 4.1 & 1.5 & 9.4 & G634 & 1684 & ENIO \\
\hline 258 & 42 & .71 & 118 & 35 & 1.9 & .21 & -17.5 & 0.11 & 3.0 & $-160 \cdot 3$ & 3.4 & B124 & 101684 & DENIO \\
\hline 8259 & 42 & 9.49 & 118 & 34 & 4163.0 & & & 0.01 & 1.0 & -173.8 & -10.9 & G534 & 1684 & ENIC \\
\hline 60 & 42 & 47 & 18 & & 1.0 & & & & 0.6 & & 5.2 & 34 & & 110 \\
\hline P261 & 42 & 9.17 & 118 & 31.76 & $417 E .0$ & 7.08 & -31.9 & $0.0 f$ & 0.6 & 5.1 & -12.2 & G534 & 1684 & ENIO \\
\hline P262 & 42 & 9.05 & 118 & 30.30 & 4232.0 & 3.52 & .30 .2 & .01 & 0.6 & -175.3 & -12.3 & 6534 & 684 & ENIO \\
\hline 263 & 42 & .32 & 118 & 27.23 & $\$ 358.0$ & 3.24 & -14.1 & .16 & 2.2 & -161.8 & 1.2 & 8124 & 684 & DENIIO \\
\hline 264 & 42 & 10.32 & 118 & 25.89 & 5.0 & .49 & -8.2 & If & 3.3 & & 4.3 & 6634 & 684 & ENIO \\
\hline & 12 & & 118 & 24 & 1.5 & 56 & -12.8 & $1 f$ & 0.9 & & -6.8 & 8124 & 684 & DERIO \\
\hline P266 & 42 & 1.29 & 118 & 22 & .4 & .01 & -0.2 & 14 & 2.3 & .5 & 3.3 & B 123 & 684 & INIO \\
\hline 267 & 42 & 12.49 & 118 & 20.80 & 7.8 & .08 & -12.9 & .15 & 0.8 & & 10.6 & 8124 & 684 & ENIO \\
\hline 268 & 42 & 02 & 118 & 19 & & & & $1 f$ & 0.5 & & & 8124 & 884 & NIU \\
\hline P269 & 42 & & 118 & 18.46 & & & & $0.1 f$ & 0.5 & -174.8 & & B124 & & DENIO \\
\hline & 42 & & 118 & & & & & & .7 & & -2.3 & & & NIO \\
\hline 71 & 42 & 63 & 118 & & 3.5 & 43 & 2.5 & $.0 f$ & 0.3 & & 9.6 & N124 & 684 & ENIO \\
\hline & 42 & 79 & 118 & & & & 4.2 & & 0.3 & & 3.3 & B 123 & & ENIO \\
\hline & 42 & & 118 & & & & 1.4 & & 0.3 & & 10.8 & $\$ 124$ & 84 & DNIO \\
\hline P274 & 42 & 17.67 & 118 & & $\$ 70.5$ & & -1.4 & of & 0.4 & & 6.1 & N124 & 101684 & DenIo \\
\hline 8275 & 42 & 16.66 & 118 & & 4519.8 & & -8.8 & 0.01 & 0.4 & & -2.6 & $N 124$ & 101684 & DENIO \\
\hline 1276 & 42 & 15.80 & 118 & 15.30 & $\$ 579.0$ & 979926.06 & -15.2 & $0.1 f$ & 0.5 & .2 & 10.5 & $\mathbf{m} 24$ & 101684 & BNIO \\
\hline דרכי & 42 & & 118 & 15.01 & 4645.8 & 979917.15 & -96.7 & $.1 f$ & 0.5 & 5.0 & -13. & N124 & 101684 & Eniso \\
\hline & 42 & & 118 & 14.16 & 4718.1 & 979918.11 & & $0.0 f$ & 0.0 & & & N124 & 101684 & ENIO \\
\hline P279 & 42 & 18.05 & 118 & 13.29 & 4856.6 & 979910.99 & -1.5 & 0.16 & 0.7 & -167.9 & & N124 & 10168 & DENI \\
\hline
\end{tabular}


sable $7-$-(continued)

\begin{tabular}{|c|c|c|c|c|c|c|c|c|c|c|c|c|c|c|c|}
\hline \multirow{2}{*}{$\begin{array}{c}\text { STATIOI: } \\
\text { WANE }\end{array}$} & \multicolumn{2}{|c|}{ LATITUDL } & \multicolumn{2}{|c|}{ LONGITUDE } & \multirow{2}{*}{$\begin{array}{l}\text { ADV } \\
\text { FExT }\end{array}$} & \multirow{2}{*}{$\begin{array}{l}\text { Oss GRuV } \\
\text { nGul }\end{array}$} & \multirow{2}{*}{$\begin{array}{l}\text { FREE } \\
\text { AIR }\end{array}$} & \multicolumn{2}{|c|}{ TRRRAIN } & \multirow{2}{*}{$\begin{array}{l}\text { BOUG } \\
\text { NWOY }\end{array}$} & \multirow{2}{*}{$\begin{array}{l}\text { Isos } \\
\text { way }\end{array}$} & \multirow{2}{*}{$\begin{array}{l}\text { ACC } \\
\text { CODE }\end{array}$} & \multirow{2}{*}{\multicolumn{2}{|c|}{$\begin{array}{r}\text { DATE } \\
M-D-Y\end{array}$}} & \multirow{2}{*}{$\begin{array}{l}\text { BASE } \\
\text { MAME }\end{array}$} \\
\hline & DLC & ; MIN & UEG & MIN & & & & EAND & cosp & & & & & & \\
\hline $\mathbf{P 2 8 0}$ & 42 & 13.68 & 118 & $12 \cdot 43$ & 4954.6 & 979904.40 & 1.6 & 0.08 & 0.6 & -168.1 & -5.4 & N124 & 101 & 1684 & DENIO \\
\hline P281 & 42 & 13.40 & 118 & 11.51 & 4953.8 & 979903.79 & 1.4 & $0.1 f$ & 0.8 & -168.2 & -5.3 & N123 & & 1086 & $M C D E$ \\
\hline$P 648$ & 42 & 0.89 & 118 & 6.12 & 5219.0 & 979870.27 & 11.5 & $0.1 f$ & 1.0 & -166.9 & -0.7 & 6634 & 8 & 686 & MCDE \\
\hline$P 649$ & 42 & 2.52 & 118 & 7.82 & 5782.3 & 979844.76 & 36.5 & 0.18 & 1.6 & -160.6 & 5.3 & $\mathbf{N 2 2}$ & 8 & 686 & MCDE \\
\hline P650 & 42 & 3.14 & 118 & 8.55 & 6077.2 & 979827.73 & 46.3 & $0.2 f$ & $2 \cdot 3$ & -160.3 & 5.4 & N224 & 8 & 686 & MCDE \\
\hline P651 & 42 & 3.72 & 118 & 9.05 & 6353.0 & 979811.65 & 55.2 & $0.5 f$ & 3.2 & -159.7 & 5.7 & N224 & 8 & 686 & MCDE \\
\hline P652 & 42 & 2.31 & 117 & 48.49 & 4450.0 & 979914.25 & -18.9 & 0.01 & 0.7 & -171.3 & -3.7 & 6634 & 8 & 686 & MCDE \\
\hline 1053 & 42 & 3.14 & 117 & 48.81 & 4452.0 & 979909.92 & -24.3 & 0.08 & 0.9 & -176.6 & -9.2 & $c 634$ & 8 & 686 & MCLE \\
\hline P654 & 42 & 5.14 & 117 & 49.91 & 4581.0 & 979907.82 & -17.3 & $0.0 f$ & 1.1 & -173.7 & -6.8 & 6634 & 8 & 686 & $M C D E$ \\
\hline$P 655$ & 42 & 5.86 & 117 & 50.39 & 4673.0 & 979904.59 & -12.8 & $0.0 f$ & 1.1 & -172.4 & -5.7 & 6634 & 8 & 686 & $M C D E$ \\
\hline P656 & 42 & 6.56 & 117 & 51.00 & 4773.0 & 979899.76 & -9.4 & $0.0 f$ & 1.3 & -172.3 & -5.7 & 6634 & 8 & 686 & MCDE \\
\hline$P 657$ & 42 & 7.36 & 117 & 51.59 & $\$ 8.62 .0$ & 979895.19 & -6.8 & 0.16 & 9.7 & -172.3 & -6.1 & 6634 & 8 & 686 & MCDE \\
\hline P658 & 42 & 7.66 & 117 & 50.50 & 4701.0 & 979904.84 & -12.7 & $0.0 f$ & 1.1 & -173.4 & -7.1 & 6644 & 8 & 686 & $M C D E$ \\
\hline Po59 & 42 & 8.64 & 117 & $\$ 8.02$ & 4531.0 & 979908.78 & -26.2 & $0.0 f$ & 0.6 & -181.6 & -15.1 & 6634 & 8 & 686 & $M C D E$ \\
\hline P660 & 42 & 9.22 & 117 & 45.25 & 4560.4 & 979922.50 & -10.6 & $0.0 f$ & 0.9 & -166.6 & -0.1 & N224 & 8 & 686 & MCDE \\
\hline$P 661$ & 42 & 8.39 & 117 & 44.97 & 4609.7 & 979918.56 & -9.4 & $0.0 f$ & 0.8 & -166.9 & -0.1 & N324 & 8 & 686 & MCDE \\
\hline$P 662$ & 42 & 7.28 & 117 & 44.66 & 4590.8 & 979915.61 & -11.8 & $0.0 f$ & 0.7 & -169.0 & -1.9 & N324 & 8 & 686 & $M C D E$ \\
\hline$P 663$ & 42 & 2.78 & 117 & 52.42 & 5927.0 & 979823.67 & 28.6 & $0.4 f$ & 3.1 & -171.9 & -5.3 & G634 & 8 & 886 & MCDE \\
\hline P664 & 42 & 2.06 & 117 & 53.42 & 5457.0 & 979848.94 & 10.8 & $0.1 f$ & 1.3 & -175.5 & -8.7 & 6634 & 8 & 886 & $M C D E$ \\
\hline$P 665$ & 42 & 2.70 & 117 & 54.38 & 5672.0 & 979837.98 & 19.1 & $1.3 f$ & 3.0 & -172.8 & -6.3 & 6644 & 8 & 886 & MCDE \\
\hline P666 & 42 & 2.99 & 117 & 56.31 & 5444.0 & 55.39 & 14.7 & $0.0 f$ & 1.2 & -171.3 & -4.8 & 6634 & 8 & 886 & $\mathrm{MCDL}$ \\
\hline 1667 & 42 & 3.63 & 117 & 57.03 & 5515.0 & 979847.89 & 12.9 & 0.11 & 1.4 & -175.3 & -8.9 & 6634 & 8 & 886 & HCDE \\
\hline P668 & 42 & 3.57 & 117 & 58.53 & 5574.0 & 979841.93 & 12.5 & $1.7 f$ & 2.9 & -176.1 & -9.9 & G644 & 8 & 886 & MCDE \\
\hline P669 & 42 & 3.74 & 117 & 59.58 & 5851.0 & 979822.35 & 18.7 & $0.6 f$ & 2.8 & 9.6 & -13.4 & 6654 & 8 & 886 & MCDE \\
\hline$P 670$ & 42 & 4.45 & 118 & 0.19 & 5633.0 & 979835.71 & 10.6 & $0.1 f$ & 1.5 & -181.5 & $-15 \cdot 5$ & 6644 & 8 & 886 & MCLE \\
\hline P671 & 42 & 4.00 & 118 & 1.52 & 5603.0 & 979835.69 & 8.4 & $0.2 f$ & 1.6 & -182.6 & -16.5 & 6644 & 8 & 886 & MCDE \\
\hline$P 672$ & 42 & 74 & 118 & 1.94 & 5225.0 & 860.58 & -0.4 & $0.1 f$ & 0.9 & 9.1 & -12.8 & 0634 & 8 & 886 & $\mathrm{MCDE}$ \\
\hline 8073 & 42 & 3.65 & 118 & 2.86 & 5327.0 & 979854.13 & 1.4 & 0.11 & 1.1 & -180.6 & -16.4 & 6634 & 8 & 886 & MCEE \\
\hline P674 & 42 & 4.91 & 118 & 4.06 & 6031.0 & 979820.41 & 32.0 & $0.8 f$ & 3.0 & -172.3 & -6.7 & 6644 & 8 & 886 & $M C D E$ \\
\hline P675 & 42 & 1.87 & 118 & 3.47 & 5106.0 & 979870.25 & -0.6 & $0.1 f$ & 0.8 & -175.4 & -9.1 & 6644 & 8 & 886 & $M C E L$ \\
\hline P676 & 42 & 4.35 & 118 & 9.42 & 6719.8 & 979788.86 & 66.0 & $0.5 f$ & 4.7 & -160.0 & 5.1 & N224 & 8 & 886 & $M C D E$ \\
\hline 1677 & 42 & 4.99 & 118 & 11.11 & 6828.0 & 82.03 & 68.3 & $0.9 f$ & 4.9 & 1.1 & 3.7 & 56 & 8 & 886 & MCDE \\
\hline Pó78 & 42 & 4.44 & 118 & 12.45 & 7115.0 & 979758.62 & 72.7 & $1.5 f$ & 6.4 & -165.1 & -0.3 & G744 & 8 & 886 & $M C D E$ \\
\hline 12679 & 42 & 5.19 & 118 & 8.73 & 6553.6 & 979800.35 & 60.6 & $0.1 f$ & 2.8 & -161.7 & 3.4 & N223 & & 1086 & $M C D E$ \\
\hline$P 680$ & 42 & 6.03 & 118 & 7.14 & 6445.0 & 979806.27 & 55.0 & 0.16 & $2 \cdot 3$ & -164.0 & 1.2 & G754 & 8 & 986 & MCDE \\
\hline P68 1 & 42 & 6.81 & 118 & 5.99 & 6475.0 & 979809.68 & 52.1 & $0.2 f$ & 2.3 & -167.9 & -3.0 & 6634 & 8 & 986 & MCDE \\
\hline P682 & 42 & 7.75 & 118 & 4.63 & 7025.0 & 979762.44 & 63.1 & $0.2 f$ & 4.0 & -174.0 & -9.4 & 67 33 & 8 & 986 & $\mathrm{MCDE}$ \\
\hline 968 & 42 & 9.60 & 118 & 5.89 & 6682.0 & 979791.57 & 57.3 & $0.2 f$ & 2.9 & -169.2 & -5.1 & G734 & 8 & 986 & $M C D E$ \\
\hline$P 684$ & 42 & 11.29 & 118 & 5.99 & 6547.0 & 979800.18 & 50.6 & 0.11 & 2.7 & -171.5 & -7.8 & G754 & 8 & 986 & MCDE \\
\hline P685 & 42 & 9.72 & 118 & 3.80 & 6804.0 & 979776.43 & 53.8 & $0.5 f$ & 3.1 & -176.9 & -12.6 & 6744 & $\mathbf{B}$ & 986 & MCDE \\
\hline P686 & 42 & 10.79 & 118 & 4.09 & 6667.0 & 979786.54 & 49.0 & $0.5 f$ & 2.8 & -177.0 & $-13 \cdot 1$ & G734 & 8 & 986 & MCDE \\
\hline 8687 & 42 & 11.94 & 118 & 4.09 & 6670.0 & 979789.09 & 50.1 & 0.11 & 3.2 & -175.7 & -12.1 & 6734 & B & 986 & $M C D E$ \\
\hline P688 & 42 & 12.73 & 118 & 4.12 & 6548.0 & 979796.40 & 4.8 & $1.7 f$ & 5.0 & -175.0 & -11.8 & 6744 & 8 & 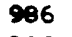 & MCDE \\
\hline 1789 & 42 & 7.40 & 118 & 2.05 & 7145.0 & 979745.81 & 58.3 & 0.31 & 4.4 & -182.5 & -17.7 & 6634 & 8 & 986 & MCDE \\
\hline 2690 & 42 & 7.02 & 118 & 1.12 & 7238.0 & 979736.78 & 58.6 & $0.7 f$ & 5.7 & -184.1 & -19.1 & 6644 & $\theta$ & 996 & MCDE \\
\hline 2691 & 42 & 7.12 & 117 & 59.25 & 7688.0 & 979707.71 & 71.6 & $0.2 f$ & 7.9 & -184.2 & -19.3 & G754 & 8 & 986 & MCDE \\
\hline 1692 & 42 & 7.05 & 117 & 57.48 & 7846.0 & 979699.36 & 78.2 & $0.9 f$ & 10.8 & -180.0 & -15.1 & 6754 & 8 & 986 & MCDE \\
\hline 2693 & 42 & 6.90 & 117 & 56.20 & 0013.0 & 979689.06 & 83.8 & $3.2 f$ & 15.8 & -175.1 & -10.1 & 6744 & 8 & 986 & MCDE \\
\hline 1694 & 42 & 8. 44 & 117 & 59.55 & 7493.0 & 979722.30 & 65.9 & $0.4 f$ & 6.1 & -185.1 & -20.4 & 6744 & $\mathbf{\theta}$ & 986 & MCDE \\
\hline 1995 & 42 & 9.26 & 117 & 59.81 & 7414.0 & 979728.65 & 63.6 & $0.6 f$ & 5.9 & -184.8 & -20.4 & 6744 & 8 & 986 & $M C D E$ \\
\hline
\end{tabular}


Iable 7--(continued)

\begin{tabular}{|c|c|c|c|c|c|c|c|c|c|c|c|c|c|c|}
\hline \multirow{2}{*}{$\begin{array}{c}\text { S2KTION } \\
\text { Wutat }\end{array}$} & \multicolumn{2}{|c|}{ LATITUDE } & \multicolumn{2}{|c|}{ LONGITUDE } & \multirow{2}{*}{$\begin{array}{l}\text { ALEV } \\
\text { TEET }\end{array}$} & \multirow{2}{*}{$\begin{array}{l}\text { Oss GRV } \\
\text { MahL }\end{array}$} & \multirow{2}{*}{$\begin{array}{l}\operatorname{Tr} x \\
\operatorname{MIR}\end{array}$} & \multicolumn{2}{|c|}{ TRRAIN } & \multirow{2}{*}{$\begin{array}{l}\text { Doug } \\
\text { Nay }\end{array}$} & \multirow{2}{*}{$\begin{array}{l}\text { Isos } \\
\text { avar }\end{array}$} & \multirow{2}{*}{$\begin{array}{l}\text { ACC } \\
\text { CODE }\end{array}$} & \multirow{2}{*}{$\begin{array}{r}\text { Dxz } \\
x-D-Y\end{array}$} & \multirow{2}{*}{$\begin{array}{l}\text { BASE } \\
\text { WME }\end{array}$} \\
\hline & DEG & $G \mathrm{MIN}$ & DEG & MIN & & & & END & cosp & & & & & \\
\hline 2696 & 42 & 12.46 & 118 & 1.01 & 7201.0 & 979748.20 & 38.4 & 0.71 & 7.0 & -181.8 & -18.4 & 6743 & 81186 & MCDE \\
\hline 8697 & 42 & 15.16 & 117 & 58.98 & 5460.0 & 979865.99 & B.5 & $0.2 f$ & $2 \cdot 2$ & -177.0 & -14.0 & 6733 & 91286 & MCDE \\
\hline 2698 & 42 & 15.40 & 117 & 57.15 & 5014.0 & 979894.04 & -5.7 & 0.08 & 1.1 & -177.1 & -14.0 & 6734 & 8996 & MCDE \\
\hline P699 & 42 & 14.40 & 177 & 54.65 & 4949.0 & 979903.44 & -0.9 & 0.08 & 0.8 & -170.4 & -6.6 & 6634 & B 986 & MCDE \\
\hline P700 & 42 & 12.85 & 117 & 46.05 & 4773.0 & 979921.53 & 2.9 & 0.01 & 0.5 & -160.7 & 4.5 & 1222 & 81686 & MCDE \\
\hline P701 & 42 & 0.30 & 118 & 7.58 & 5532.0 & 979849.90 & 21.5 & 0.11 & 1.2 & -167.5 & -1.4 & 6744 & 81086 & HCDE \\
\hline P702 & 42 & 0.07 & 118 & 5.17 & 5224.0 & 979864.75 & 7.7 & 0.11 & 0.9 & -171.0 & -4.7 & 6744 & 81086 & MCDE \\
\hline P703 & 42 & 5.93 & 118 & 8.64 & 6532.3 & 979802.47 & 59.6 & 0.21 & 2.7 & -162.0 & 3.0 & K224 & 81086 & MCDE \\
\hline P704 & 42 & 6.65 & 118 & 8.97 & 6473.1 & 979807.67 & 58.2 & 0.21 & 2.5 & -161.6 & 3.2 & 1224 & 81086 & MCDE \\
\hline P705 & 42 & 7.23 & 118 & 9.09 & 6410.1 & 979812.14 & 55.8 & 0.16 & 2.2 & -162.1 & 2.6 & N224 & 81086 & MCDE \\
\hline P706 & 42 & 7.93 & 118 & 8.64 & 6322.0 & 979817.35 & 51.7 & $0.0 f$ & 1.8 & -163.6 & 0.9 & C644 & 81086 & MCDE \\
\hline 2707 & 42 & 9.21 & 118 & 7.95 & 6150.0 & 979828.27 & 44.5 & 0.15 & 1.6 & -165.1 & -0.8 & 6654 & 81086 & $M C D E$ \\
\hline P708 & 42 & 7.98 & 118 & 9.66 & 6346.5 & 979816.45 & 53.0 & $0.0 f$ & 2.4 & -162.5 & 2.0 & B 124 & 81086 & MCDE \\
\hline P709 & 42 & 6.91 & 118 & 9.82 & 6182.5 & 979026.63 & 46.4 & 0.28 & 2.2 & -163.8 & 0.5 & 1224 & 81086 & MCDE \\
\hline P7 10 & 42 & 9.84 & 118 & 9.94 & 6076.9 & 979833.53 & 42.0 & $0.2 f$ & 2.1 & -164.7 & -0.6 & B 124 & 81086 & MCUE \\
\hline 5711 & 42 & 10.30 & 118 & 10.41 & 5889.9 & 979846.02 & 36.2 & $0.4 f$ & 1.9 & -164.2 & -0.3 & B124 & 81086 & MCLE \\
\hline 8712 & 42 & 11.01 & 118 & 10.89 & 5485.7 & 979872.62 & 23.8 & 0.18 & 1.1 & -163.6 & 0.1 & N224 & 81086 & MCUE \\
\hline 4713 & 42 & 11.86 & 118 & 10.97 & 5301.8 & 979884.53 & 17.1 & $0.1 f$ & 1.0 & -164.1 & -0.6 & 1224 & 81086 & $M C D E$ \\
\hline P714 & 42 & 12.74 & 118 & 11.09 & 5114.7 & $979894 \cdot 19$ & 7.9 & $0.2 f$ & 1.0 & -167.0 & -3.8 & N224 & 81086 & MCDE \\
\hline 2715 & $4<$ & 14.07 & 116 & 10.92 & 4826.0 & 979914.77 & -0.7 & $0.6 f$ & 1.4 & -165.3 & -2.5 & 6744 & 81086 & MCDE \\
\hline P7 16 & 42 & 12.73 & 118 & 13.81 & 4935.0 & 979904.77 & 1.6 & $0.2 f$ & 0.9 & -167.3 & -4.2 & G634 & 81086 & MCDE \\
\hline P717 & 42 & 11.82 & 118 & 13.75 & 5207.0 & 979887.29 & 11.0 & 0.18 & 1.0 & -167.0 & -3.7 & C644 & 81086 & MCDE \\
\hline P7 18 & 42 & 11.06 & 118 & 15.21 & 5416.0 & 979879.46 & 24.0 & $0.3 f$ & 1.5 & -160.7 & 2.7 & G754 & 81086 & MCUE \\
\hline 1719 & 42 & 11.14 & 118 & 18.30 & 5221.0 & 979884.95 & 11.0 & 0.11 & 1.2 & -167.3 & -4.2 & 6634 & 81086 & $M C D E$ \\
\hline 8720 & 42 & 11.64 & 118 & 20.39 & 4937.0 & 979898.44 & -2.9 & $0.0 f$ & 0.8 & -171.9 & -9.1 & G633 & 81886 & DENIO \\
\hline 2721 & 42 & 12.59 & 118 & 17.68 & 4774.0 & 979905.50 & -12.6 & $0.1 f$ & 0.8 & -176.0 & $-13 \cdot 3$ & 6634 & 81086 & $M C D E$ \\
\hline P722 & 42 & 13.87 & 118 & 18.25 & 4646.0 & 979914.65 & -17.4 & $0.0 f$ & 0.5 & -176.8 & -14.5 & 6634 & 81086 & MCDE \\
\hline P7 23 & 42 & $1+.25$ & 118 & 16.77 & 4630.0 & 979913.94 & -20.2 & $0.0 f$ & 0.5 & -179.0 & -16.8 & 6754 & 81066 & MCDE \\
\hline P724 & 42 & 28.38 & 117 & 52.98 & 4340.0 & 979986.72 & 4.1 & $0.1 f$ & 0.3 & -144.9 & 11.5 & G644 & 81086. & MCDE \\
\hline P725 & 42 & 9.55 & 118 & 0.75 & 7129.0 & 979750.90 & 58.7 & $0.3 f$ & 3.7 & -182.3 & -17.9 & G744 & 81186 & MCDE \\
\hline P726 & 42 & 10.58 & 118 & 1.62 & 7088.0 & 979756.67 & 59.0 & $0.1 f$ & 3.6 & -180.6 & -16.6 & 6744 & 81186 & MCDE \\
\hline 8727 & 42 & 11.30 & 118 & 0.51 & 7348.0 & 979735.67 & 61.4 & 1.91 & 8.7 & -182.0 & -18.3 & 6744 & 81186 & MCDE \\
\hline P728 & 42 & 13.52 & 118 & 0.99 & 6968.0 & 979765.26 & 51.9 & 0.61 & 5.9 & -181.3 & -18.3 & 6734 & 81186 & MCDE \\
\hline$F 729$ & 42 & 14.31 & 118 & 3.22 & 6763.0 & 979788.30 & 54.5 & $0.2 f$ & 3.4 & -174.2 & -11.6 & G754 & 81186 & MCDE \\
\hline P730 & 42 & 14.22 & 118 & 5.84 & 6385.0 & 979813.27 & 44.1 & $1.1 f$ & 4.7 & -170.5 & -7.9 & 6734 & 81186 & $M C D E$ \\
\hline 2731 & 42 & 15.96 & 118 & 4.67 & 6382.0 & 979819.96 & 47.9 & 0.11 & 2.6 & -168.7 & -6.8 & 6744 & 81186 & $M C D E$ \\
\hline P732 & 42 & 16.42 & 118 & 7.03 & 5683.0 & 979873.16 & 34.7 & $0.2 f$ & 1.7 & -158.9 & 2.8 & 6634 & 81186 & MCDE \\
\hline P733 & 42 & 17.22 & 118 & 7.01 & 5584.0 & 979880.83 & 31.9 & $0.2 f$ & 1.6 & -158.5 & 2.9 & C644 & 81186 & MCDE \\
\hline P734 & 42 & $18 \cdot 36$ & 118 & 8.49 & 5082.0 & 979917.43 & 19.6 & $0.1 f$ & 0.9 & -154.3 & 6.6 & 6634 & 81186 & MCDE \\
\hline 2735 & 42 & 19.17 & 118 & 10.11 & 4814.0 & 979935.88 & 18.6 & $0.1 f$ & 0.6 & -153.3 & 7.1 & 6634 & 81186 & $\mathrm{MCDL}$ \\
\hline P736 & 42 & 18.00 & 118 & 10.75 & 4758.0 & 979935.87 & 0. 1 & 0.15 & 0.6 & -154.9 & 6.1 & G754 & 81186 & MCDE \\
\hline P737 & 42 & 17.33 & 118 & 11.57 & 1643.0 & 979939.42 & 1.9 & 0.31 & 0.9 & -156.9 & 4.4 & G744 & B1186 & MCDE \\
\hline $\begin{array}{l}P 738 \\
2739\end{array}$ & $\begin{array}{l}42 \\
42\end{array}$ & $\begin{array}{l}18.38 \\
19.55\end{array}$ & $\begin{array}{l}118 \\
118\end{array}$ & $\begin{array}{l}12.35 \\
12.91\end{array}$ & $\begin{array}{r}4785.0 \\
4459.0\end{array}$ & $\begin{array}{l}979932.24 \\
979960.07\end{array}$ & $\begin{array}{l}6.5 \\
1.9\end{array}$ & $\begin{array}{l}0.41 \\
0.11\end{array}$ & $\begin{array}{l}0.9 \\
0.4\end{array}$ & $\begin{array}{l}-157.2 \\
-151.1\end{array}$ & $\begin{array}{l}3.5 \\
9.1\end{array}$ & $\begin{array}{l}6644 \\
6634\end{array}$ & $\begin{array}{l}81186 \\
81186\end{array}$ & $\begin{array}{l}\text { MCDE } \\
\text { MCDE }\end{array}$ \\
\hline P740 & 42 & 22.09 & 118 & 10.35 & 4466.0 & 979963.23 & 1.9 & 0.08 & 0.3 & -151.5 & 7.7 & 6634 & 81186 & MCDE \\
\hline 2741 & 42 & 21.35 & 118 & 9.36 & 4576.0 & 979956.26 & 6.4 & $0.0 f$ & 0.5 & -150.6 & 0.9 & 6634 & 81186 & MCDE \\
\hline$P 742$ & 42 & 15.77 & 118 & 1.52 & 7353.0 & 979749.18 & 68.7 & $0.8 f$ & 8.8 & -174.8 & -12.9 & $\sqrt{324}$ & 01286 & MCDE \\
\hline 2743 & 42 & 16.57 & 118 & 2.60 & 7006.0 & 979775.49 & 61.2 & $0.3 f$ & 5.7 & -173.6 & -12.1 & 6634 & 81286 & MCDE \\
\hline$P 744$ & 42 & 17.53 & 117 & 59.89 & 6936.0 & 979775.19 & 52.8 & 2.96 & 11.9 & $-173 \cdot 4$ & -12.1 & 674 & 81286 & MCDE \\
\hline$P 745$ & 42 & 16.82 & 117 & 57.28 & 4974.0 & 979900.76 & -4.4 & 0.01 & 1.1 & -174.6 & -12.1 & 6734 & 81286 & MCDE \\
\hline
\end{tabular}


gable 7--(continued)

\begin{tabular}{|c|c|c|c|c|c|c|c|c|c|c|c|c|c|c|}
\hline & & & LONG & GITUDE & & OBS GRAV & FREE & TERP & IIN & DOUG & Isos & ncc & DATE & BASE \\
\hline waMk & DEG & MIN & USE & MIN & FEET & MGAL & AIR & HAND & comp & NaY & Mar & CODE & $M-D-X$ & NAML \\
\hline P746 & 42 & 17.60 & 117 & 58.09 & 5042.0 & 979895.23 & -5.2 & 0.11 & 1.6 & -177.0 & -15.0 & 6734 & 81286 & MCOE \\
\hline 2747 & 42 & 18.83 & 117 & 58.18 & 5110.0 & 979896.80 & 0.9 & $0.1 f$ & 1.3 & -173.5 & -12.1 & 6734 & 81286 & MCDE \\
\hline$P 748$ & 42 & $20 \cdot 10$ & 117 & 58.80 & 5281.0 & 979891.61 & 9.9 & $0.5 f$ & 1.5 & -170.2 & -9.7 & $\cos 4$ & 81286 & $M C D E$ \\
\hline 8749 & 42 & 19.28 & 117 & 59.22 & 5349.3 & 979882.79 & 0.7 & 0.26 & 1.7 & -173.5 & -12.6 & 4224 & 81286 & MCDE \\
\hline$P 750$ & 42 & 21.24 & 118 & 0.39 & 5081.1 & 979913.82 & 11.6 & $0.5 f$ & 1.5 & -161.7 & -1.8 & 8224 & 81286 & MCDE \\
\hline P751 & 42 & 22.55 & 118 & 1.46 & 4941.1 & 979931.77 & 14.4 & $0.2 f$ & 0.8 & -154.7 & 4.4 & 2022 & 81286 & $M C D L$ \\
\hline P752 & 42 & 21.81 & 118 & 2.34 & 4923.0 & 979930.89 & 12.9 & 0.08 & 0.8 & -155.6 & 3.9 & 6634 & 81286 & MCDE \\
\hline 8753 & 42 & 23.13 & 118 & 3.13 & 4662.0 & 979954.10 & 9.6 & 0.11 & 0.6 & -150.2 & 8.6 & 6634 & 81286 & $M C D E$ \\
\hline P754 & 42 & 24.30 & 118 & 3.48 & 4495.0 & 979969.33 & 7.4 & $0.0 f$ & 0.3 & -146.9 & 11.3 & 6634 & 81286 & MCDE \\
\hline P755 & 42 & $25.5 C$ & 118 & 4.05 & 4388.0 & 979901.04 & 7.3 & 0.01 & 0.2 & -143.5 & 14.1 & 6634 & 81286 & MCDE \\
\hline P756 & 42 & 24.54 & 118 & 6.05 & 4368.0 & 979976.80 & 2.6 & $0.0 f$ & 0.3 & -147.5 & 10.6 & 6534 & 81286 & MCDE \\
\hline 1757 & 42 & 23.32 & 118 & 8.30 & 4462.6 & 979970.59 & 7.1 & $0.0 f$ & 0.3 & -146.1 & 12.5 & $\mathrm{N224}$ & 81286 & MCDE \\
\hline P758 & 42 & 21.82 & 138 & 6.99 & 4790.0 & 979946.72 & 16.3 & $0.1 f$ & 0.7 & -147.8 & 11.5 & 6634 & 81286 & MCDE \\
\hline P759 & 42 & 25.89 & 118 & 2.33 & 4686.0 & 979961.55 & 15.2 & $0.0 f$ & 0.4 & -145.6 & 11.7 & C634 & 81286 & $M C D E$ \\
\hline 8760 & 42 & 12.89 & 117 & 48.22 & $468 B .0$ & 979925.68 & -1.0 & 0.08 & 0.5 & -161.7 & 3.2 & 6634 & 81286 & $M C D E$ \\
\hline P761 & 42 & 13.01 & 117 & 50.82 & $\$ 725.0$ & 979913.24 & $-10 \cdot 1$ & $0.0 \mathrm{f}$ & 0.6 & -172.1 & -7.4 & 6634 & 81286 & MCUI \\
\hline P762 & 42 & 13.54 & 117 & 52.54 & 4823.0 & 979909.82 & -5.1 & $0.0 f$ & 0.6 & -170.4 & -6.1 & 6534 & 81286 & $M C D E$ \\
\hline 8763 & 42 & 14.12 & 117 & 56.52 & 5053.0 & 979886.93 & -5.3 & $0.0 f$ & 1.1 & -177.9 & -14.2 & 6634 & 81286 & MCDE \\
\hline P764 & 42 & 13.89 & 117 & 57.76 & 5148.0 & 979880.10 & $-4 \cdot 8$ & $0.0 f$ & 1.6 & -180.2 & -16.5 & 6634 & 81286 & $M C D E$ \\
\hline 2765 & 42 & 13.22 & 117 & 38.38 & 6246.1 & 979829.57 & 48.9 & $0.3 f$ & 1.9 & -163.8 & 1.8 & B223 & 81486 & $M C D E$ \\
\hline 8766 & 42 & 17.37 & 117 & 34.42 & 5537.5 & 979877.84 & 24.3 & 0.08 & 0.5 & -165.5 & -1.0 & $N 224$ & B1386 & $M C D E$ \\
\hline 2767 & 42 & 15.73 & 117 & 30.96 & 5607.4 & 979877.92 & 33.4 & $0.1 f$ & 0.5 & -158.8 & 6.9 & B124 & 81386 & $\mathrm{BCDE}$ \\
\hline P768 & 42 & 14.37 & 117 & 30.01 & 5666.4 & 979871.95 & 35.0 & $0.0 f$ & 0.6 & -159.1 & 7.3 & B124 & B1386 & MCDE \\
\hline 5769 & 42 & 11.87 & 117 & 28.91 & 6043.3 & 979845.82 & 48.1 & $0.0 f$ & 0.8 & -158.7 & 8.9 & 5124 & 81386 & MCDE \\
\hline P770 & 42 & 11.11 & 117 & 28.09 & 6088.9 & 979842.19 & 49.9 & $0.0 f$ & 0.9 & -158.4 & 9.5 & 8124 & 81386 & MCDE \\
\hline 8771 & 42 & 10.43 & 117 & 27.55 & 6136.9 & 979837.89 & 51.1 & $0.0 f$ & 0.9 & -158.8 & 9.4 & B124 & 81386 & $\triangle C D E$ \\
\hline P772 & 42 & 9.75 & 117 & 26.76 & 6210.2 & 979831.77 & 52.9 & $0.0 f$ & 1.0 & -159.4 & 9.1 & B124 & 81386 & MCDE \\
\hline P773 & 42 & 9.62 & 117 & 25.38 & 6232.0 & 979828.96 & 52.3 & 0.11 & 1.1 & -160.6 & 8.2 & B123 & 81486 & MCDE \\
\hline P774 & 42 & 9.33 & 117 & $24 \cdot 10$ & 6141.0 & 979834.93 & 50.2 & $0.1 f$ & 1.0 & -159.8 & 9.2 & B 124 & 81386 & $M C D E$ \\
\hline P775 & 42 & 9.17 & 117 & 22.98 & 6051.7 & 979839.25 & 46.3 & $0.0 f$ & 0.8 & -160.7 & B. 5 & B124 & 81386 & MCDE \\
\hline P776 & 42 & 8.82 & 117 & 21.75 & 5932.4 & 979845.22 & 41.6 & 0.08 & 0.7 & -161.5 & 8.0 & 8224 & 81386 & MCDE \\
\hline 8777 & 42 & 8.80 & 117 & 20.51 & 5850.3 & 979849.20 & 37.9 & $0.0 f$ & 0.6 & -162.5 & 7.2 & B124 & 81386 & MCDE \\
\hline P778 & 42 & 8.35 & 117 & 19.28 & 5759.3 & 979852.64 & 33.5 & $0.1 f$ & 0.6 & -163.8 & 6.2 & 8124 & 81386 & $M C D E$ \\
\hline P779 & 42 & 6.19 & 117 & 18.91 & 5932.0 & 979837.31 & 37.6 & $0.0 f$ & 0.7 & -165.5 & 5.2 & 6754 & 81386 & $M C D E$ \\
\hline P780 & 42 & 5.31 & 117 & 18.68 & 5942.0 & 979833.96 & 36.5 & $0.0 f$ & 0.6 & -167.0 & 3.9 & 6634 & 81386 & MCDE \\
\hline P781 & 42 & 4.24 & 117 & 18.15 & 5996.0 & 979826.30 & 35.6 & $0.0 f$ & 0.6 & -169.8 & 1.4 & 6634 & 81386 & MCDE \\
\hline 2782 & 42 & 4.13 & 117 & 19.98 & 5935.0 & 979830.64 & 34.3 & $0.0 f$ & 0.6 & -169.0 & 2.2 & 6534 & 81386 & MCDL \\
\hline P783 & 42 & 3.54 & 117 & 21.06 & 5956.0 & 979826.59 & 33.2 & $0.0 f$ & 0.6 & -170.9 & 0.4 & 6634 & 81386 & ACDE \\
\hline 8784 & 42 & 3.35 & 117 & 22.17 & 5927.0 & 979828.09 & 32.2 & 0.11 & 0.6 & -170.8 & 0.4 & 6534 & 81386 & $M C D E$ \\
\hline 8785 & 42 & 2.40 & 117 & 23.65 & 5947.0 & 979823.68 & 31.1 & 0.01 & 0.6 & -172.6 & -1.2 & 6544 & 81386 & $M C D E$ \\
\hline P786 & 42 & 1.66 & 117 & 23.96 & 5963.0 & 979821.46 & 31.5 & 0.11 & 0.7 & -172.7 & -1.2 & 6634 & 81386 & $M C D E$ \\
\hline 2787 & 42 & 2.32 & 117 & 24.66 & 5976.0 & 979823.11 & 33.4 & 0. if & 0.7 & -171.3 & -0.1 & 0634 & 81386 & MCDE \\
\hline 2788 & 42 & 3.81 & 117 & 26.43 & 6144.0 & 979819.14 & 43.0 & 0.08 & 0.8 & $-167 \cdot 3$ & $3 \cdot 2$ & 6634 & 81386 & $\mathrm{MCDE}$ \\
\hline 2789 & 42 & 3.25 & 117 & 27.74 & 6219.0 & 979813.86 & 45.6 & 0.01 & 0.9 & -167.1 & 3.3 & 6644 & 81386 & $\mathrm{ACDL}$ \\
\hline 1790 & 42 & 3.43 & 117 & 29.49 & 6294.0 & $979810 \cdot 10$ & 48.6 & 0.01 & 1.1 & -166.5 & $3 \cdot 6$ & 6634 & 81306 & MCDE \\
\hline PS1 & 42 & 2.89 & 117 & 31.45 & 6353.0 & $97980 \mathrm{~b} .25$ & 50.1 & $0.0 f$ & 1.4 & -166.7 & 3.2 & Q734 & 81386 & MCDE \\
\hline$P 792$ & 42 & 1.39 & 117 & $33 \cdot 30$ & 6229.0 & 979812.35 & 47.8 & 0.05 & 2.2 & -163.9 & 5.8 & 6534 & 81386 & $M C D E$ \\
\hline 9793 & 42 & 0.23 & 117 & 36.48 & 4914.0 & 979894.25 & 7.8 & $0.1 f$ & 1.0 & -160.1 & 9.7 & 6634 & 81386 & nCUE \\
\hline 794 & 42 & 0.29 & 117 & 38.07 & 4742.0 & 979896.35 & -6.3 & 0.01 & 0.6 & -168.8 & 0.7 & 6634 & 81386 & MCDE \\
\hline 9795 & 42 & 13.79 & 117 & 44.52 & 4949.2 & 979919.59 & 16.1 & 0.26 & 0.8 & -153.3 & 18.7 & 1224 & 81486 & MCDE \\
\hline
\end{tabular}


Table 7--(continued)

\begin{tabular}{|c|c|c|c|c|c|c|c|c|c|c|c|c|c|c|}
\hline $\begin{array}{l}\text { TATION } \\
\text { NAMLE }\end{array}$ & $\begin{array}{l}2 A T \\
\text { DEG }\end{array}$ & SITUDE & $\begin{array}{l}\text { LONG } \\
\text { ULG }\end{array}$ & $\begin{array}{l}\text { GITUDE } \\
\text { MIN }\end{array}$ & $\begin{array}{l}\text { EREV } \\
\text { TLET }\end{array}$ & $\begin{array}{l}\text { Cos GRAv } \\
\text { MGLL }\end{array}$ & $\begin{array}{l}\text { FREE } \\
\text { AIR }\end{array}$ & $\begin{array}{l}\text { TERP } \\
\text { LAND }\end{array}$ & $\begin{array}{l}\text { AIN } \\
\text { Cunip }\end{array}$ & $\begin{array}{l}\text { DOUG } \\
\text { NWOM }\end{array}$ & $\begin{array}{l}\text { IsOS } \\
\text { MNOH }\end{array}$ & $\begin{array}{l}A C C \\
\text { CODE }\end{array}$ & $\begin{array}{r}\text { DATE } \\
M-D-Y\end{array}$ & $\begin{array}{l}\text { BASE } \\
\text { MNLL }\end{array}$ \\
\hline 8796 & 42 & 14.24 & 117 & 42.84 & 270.1 & 979892.22 & 18.3 & $0.2 f$ & 1.0 & -161.9 & 2.9 & 11224 & 81486 & MCUE \\
\hline P797 & 42 & 13.81 & 117 & 41.50 & 5842.9 & 979857.33 & 37.8 & $0.8 f$ & 2.3 & -160.6 & 4.4 & N224 & 81486 & $\mathrm{MCOL}$ \\
\hline P798 & 42 & 13.79 & 117 & 40.08 & 6283.9 & 979828.15 & 50.1 & 0.71 & 2.9 & -162.8 & 2.2 & N224 & 81486 & MCDE \\
\hline 8799 & 42 & 11.98 & 117 & 37.35 & 5932.0 & 979844.79 & 36.4 & $0.1 f$ & 0.9 & -166.4 & -0.1 & C644 & 81486 & MCDE \\
\hline P800 & 42 & 11.15 & 117 & 36.35 & 6265.0 & 979826.31 & 50.5 & $0.1 f$ & 1.6 & -163.1 & 3.5 & 6633 & 1486 & MCDE \\
\hline P80 1 & 42 & 10.25 & 117 & 34.93 & 6072.0 & 979837.61 & 45.0 & 0.02 & 1.0 & -162.6 & 4.6 & C634 & 31486 & $M C D E$ \\
\hline$P 802$ & 42 & 10.41 & 117 & 38.14 & 6414.0 & 979817.07 & 56.4 & 0.11 & 2.7 & -161.2 & 5.3 & 6644 & 1486 & MCUE \\
\hline P803 & 42 & 7.68 & 117 & 36.08 & 6464.0 & 979808.02 & 6.1 & $0.1 f$ & 2.2 & -163.7 & 4.0 & 6634 & 1486 & $M C D E$ \\
\hline 8804 & 42 & 8.73 & 117 & 36.74 & 6484.0 & 979809.21 & 57.6 & $0.0 f$ & 2.3 & -162.7 & 4.6 & 654 & 1486 & MCDE \\
\hline 5805 & 42 & 12.37 & 117 & 35.95 & 5884.0 & 979851.67 & 38.2 & $0.0 f$ & 0.8 & 3.2 & 3.2 & 6634 & 1486 & ICDE \\
\hline P806 & 42 & 13.85 & 117 & 36.82 & 5892.4 & 979850.28 & 35.4 & .16 & 0.9 & -166.2 & -0.6 & B124 & 1486 & MCUE \\
\hline 8807 & 42 & 13.52 & 117 & 29.55 & 5872.8 & 979858.21 & 42.0 & $.0 f$ & 0.7 & -159.1 & 7.7 & B124 & 1486 & $M C D E$ \\
\hline P808 & 42 & 13.66 & 117 & 27.94 & 5766.0 & 979865.99 & 39.5 & . If & 0.6 & -158.0 & 8.9 & 6634 & 1486 & MCDE \\
\hline 809 & 42 & 12.74 & 117 & 29.57 & 5989.0 & 979850.38 & 46.2 & $.1 f$ & 0.9 & -158.7 & 8.4 & B124 & 1486 & MCDE \\
\hline 310 & 42 & 12.25 & 117 & 25.59 & 5884.0 & 979856.21 & 42.9 & $0.0 f$ & 0.7 & -158.5 & 9.3 & C634 & 1486 & $M C D E$ \\
\hline 5819 & 42 & 11.55 & 117 & 23.89 & 5723.0 & 979864.04 & 36.7 & 0.11 & 0.6 & -159.4 & 8.9 & C634 & 1486 & $M C D E$ \\
\hline P812 & 42 & 7.34 & 117 & 18.05 & 5701.5 & 979853.80 & 30.7 & $0.1 f$ & 0.5 & -164.7 & 5.7 & N324 & 1486 & $M C D E$ \\
\hline 5613 & 42 & 7.06 & 117 & 15.94 & 5760.8 & 979849.18 & 2.1 & $.0 f$ & 0.4 & -165.4 & 5.1 & $N 224$ & 1486 & $M C D E$ \\
\hline PB14 & 42 & 6.81 & 117 & 13.66 & 677.1 & 979852.76 & 8.2 & .01 & 0.4 & -166.5 & 4.3 & N224 & 1486 & $\triangle C D E$ \\
\hline 1815 & 42 & 5.55 & 117 & 13.50 & 5648.8 & 0.70 & 5.4 & $.0 f$ & 0.4 & 8.4 & 2.8 & $N 224$ & 1486 & $\operatorname{COL}$ \\
\hline 816 & 42 & 3.72 & 117 & 68 & 1.8 & & .2 & Df & & & 1.7 & & & CDE \\
\hline 8817 & 42 & 2.60 & 117 & 15.81 & 5825.1 & .61 & .3 & of & .5 & -172.4 & -0.4 & N224 & 186 & $C D L$ \\
\hline P818 & 42 & 0.14 & 117 & 17.45 & 6030.4 & .36 & 1.0 & & 0.6 & -176.5 & -4.0 & N224 & 186 & CDE \\
\hline 825 & 42 & 1.04 & 117 & 43.86 & 5.0 & 97 & 5 & & .3 & 8.8 & 10.4 & 6634 & 86 & $\mathrm{CDE}$ \\
\hline 826 & 42 & 1.88 & 117 & 45.52 & 5.0 & .51 & 5 & & .3 & 0.5 & -12.5 & 6634 & 86 & ICDE \\
\hline$P 527$ & 42 & 2.99 & 117 & 43.47 & 6.0 & & -1 & & .4 & 9.4 & -1.3 & 6634 & 586 & $C D_{t}$ \\
\hline 828 & 42 & 3.87 & 117 & 45. & & & & & & .9 & -5.2 & 334 & 386 & ICJE \\
\hline 629 & 42 & 6.33 & 117 & 46.64 & 3.0 & .27 & & & .5 & & -12.0 & 6644 & 686 & $B C D L$ \\
\hline$P 830$ & 42 & 4.46 & 117 & 43.88 & 4643.0 & 1.45 & -6 . & $0 f$ & 0.4 & 6.1 & 1.7 & G634 & 31686 & $A C D E$ \\
\hline r8s 1 & 42 & 10.35 & 117 & & 4609.2 & 2.29 & & $0.0 f$ & 0.9 & & 0.5 & $N 224$ & 1686 & $\operatorname{CDE}$ \\
\hline PB32 & 42 & .20 & 17 & & 7.0 & & .7 & if & 1.2 & .2 & 1.7 & & & ICUE \\
\hline 8833 & 42 & 10.32 & 117 & 53.97 & 5273.0 & 0.23 & 2.4 & $0.2 \mathrm{f}$ & 2.5 & -176.4 & -11.2 & G634 & 11686 & $A C D E$ \\
\hline$P 834$ & 42 & 11.77 & 117 & 53.60 & 4964.0 & 979890.98 & -8.0 & 0.08 & 1.2 & -177.6 & -12.7 & 6634 & $316 B 6$ & $M C D E$ \\
\hline$P 835$ & 42 & 11.95 & 117 & 55.51 & 5206.0 & 1.29 & -2.3 & $0.1 f$ & 2.2 & -179.0 & -14.4 & 6634 & 31686 & MCDE \\
\hline 8836 & 42 & 12.41 & 17 & 56 & 5445.0 & 97 & 1.7 & $5 f$ & 2.9 & -179.5 & -15.3 & 6644 & 1686 & DENIO \\
\hline 8837 & 42 & 8.77 & 16 & 28.92 & 4346.8 & 3.49 & 24.6 & $0.0 f$ & 0.6 & -173.3 & -10.2 & $N 224$ & 1686 & DENIO \\
\hline P838 & 42 & 7.89 & 18 & 30 & 4212.0 & .23 & 30.6 & 0.08 & 0.6 & -175.0 & -11.7 & 6633 & 1886 & DENIO \\
\hline 339 & 42 & & 18 & & & & & of & 0.7 & .4 & 13.9 & & 1686 & DENIO \\
\hline$P 840$ & 42 & 4.95 & 18 & 30.32 & 4229.0 & 27 & .6 & 0.08 & 0.8 & 5.4 & -21.5 & 6634 & 1686 & DENIO \\
\hline 2841 & 42 & 3.13 & 118 & 29.89 & 4280.0 & 979909.47 & -40.9 & $0.0 f$ & 0.9 & -187.2 & -23.1 & 6634 & 81686 & DENIO \\
\hline P842 & 42 & 2.53 & 118 & 30.59 & 4218.0 & 9.65 & 45.6 & $0.0 \mathrm{f}$ & 1.0 & -189.8 & -25.6 & $\$ 424$ & & \\
\hline 843 & 42 & 1.38 & 118 & 32 & 4.0 & & & .02 & 0.9 & -1 & -26.3 & & 86 & DENIO \\
\hline 444 & 42 & 78 & 118 & 33. & 3.0 & & & $.0 f$ & 0.9 & 0.0 & -25.7 & & 186 & DENIO \\
\hline 845 & 42 & 18 & 118 & 36.46 & $\$ 179.0$ & 3.33 & -32.1 & 0.08 & 1.4 & -174.5 & -10.1 & C644 & 386 & DENIU \\
\hline 359 & 42 & 11.36 & 118 & 23 & $\$ 622.0$ & & -7.4 & $0.5 f$ & 1.7 & -164.7 & -2.0 & & 186 & DENIO \\
\hline 160 & 42 & 49 & 118 & & $y .0$ & & & 0.11 & 6.3 & -161.4 & 2.0 & 144 & 186 & DE:IIO \\
\hline P861 & 42 & 6.64 & 118 & 18.12 & 7192.0 & 979754.12 & 72.2 & 2.11 & 9.9 & -164.7 & -1.0 & 6754 & 81886 & DENIO \\
\hline 362 & 42 & 4.79 & 118 & 16.06 & 7742.0 & 979715.77 & 88.3 & $0.3 t$ & 7.8 & -169.5 & -5.4 & G754 & 81886 & UENIO \\
\hline & 42 & 4.20 & 118 & & & & & se & 8. & & & & $B 1886$ & DENIO \\
\hline 864 & 42 & .92 & 118 & 14.99 & 0041.0 & 979689.82 & 93.2 & $0.6 f$ & 8.6 & -173.9 & & 6754 & B1886 & DEAIIO \\
\hline
\end{tabular}


sable 7--(continued)

STATIUN LATITUDE LONGITUDE While NLG RII DEG MIN

$\begin{array}{lllll}\text { P865 } & 42 & 2.65 & 118 & 13.68 \\ \text { P866 } & 42 & 2.65 & 118 & 16.60 \\ \text { P667 } & 42 & 4.56 & 118 & 17.75 \\ \text { P868 } & 42 & 0.30 & 118 & 17.87 \\ \text { P869 } & 42 & 1.32 & 118 & 18.83 \\ \text { P670 } & 42 & 1.31 & 118 & 19.59 \\ \text { P871 } & 42 & 4.83 & 118 & 20.84 \\ \text { P872 } & 42 & 6.00 & 118 & 23.13 \\ \text { P673 } & 42 & 6.45 & 118 & 26.88 \\ \text { P874 } & 42 & 13.51 & 118 & 38.72\end{array}$

$\begin{array}{llllll}P 875 & 42 & 15.89 & 118 & 40.48\end{array}$ $\begin{array}{llllll}P 876 & 42 & 18.38 & 118 & 43.31\end{array}$ $\begin{array}{lllll}8877 \quad 42 & 19.05 & 118 & 44.97\end{array}$ $\begin{array}{lllll}P 878 & 42 & 20.10 & 118 & 47.09\end{array}$ $\begin{array}{lllll}P 879 & 42 & 20.49 & 118 & 48.27\end{array}$ $\begin{array}{llllll}P 880 & 42 & 19.68 & 118 & 50.35\end{array}$ $\begin{array}{lllll}\text { P881 } 42 & 18.23 & 118 & 50.74\end{array}$ $\begin{array}{lllll}P 882 & \triangle 2 & 16.91 & 118 & 50.92\end{array}$ $\begin{array}{lllll}P 883 \quad 42 & 15.56 & 118 & 52.33\end{array}$ $\begin{array}{lllll}P 884 & 42 \quad 0.51 & 118 & 52.59\end{array}$

$\begin{array}{llllll}P 885 & 42 & 1.25 & 118 & 53.57\end{array}$ $\begin{array}{lllll}1486 & 42 \quad 3.10 & 118 & 56.74\end{array}$ $P 887 \quad 42 \quad 4.09 \quad 118 \quad 57.76$ $\begin{array}{lllll}P 688 & 42 & 5.16 \quad 118 & 59.06\end{array}$ $\begin{array}{llllll}8889 & 42 & 5.82 & 119 & 0.70\end{array}$ $\begin{array}{lllll}42 & 5.66 & 115 & 2.00\end{array}$ $\begin{array}{lllll}\text { P89 } & 42 & 6.21 & 119 & 5.10\end{array}$ $\begin{array}{lllll}8892 & 42 & 7.65 & 119 & 4.10\end{array}$ $\begin{array}{lllll}\text { P893 } 42 & 8.30 & 119 & 3.47\end{array}$

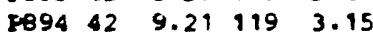

$\begin{array}{lllll}P 895 & 42 & 9.95 & 119 & 3.49\end{array}$ $\begin{array}{llllll}\text { PU9C } 42 & 10.96 & 119 & 4.01\end{array}$ $\begin{array}{lllll}P 897 \quad 42 & 11.44 & 119 & 3.19\end{array}$ $\begin{array}{llllll}\text { P498 } 42 & 11.94 & 119 & 2.25\end{array}$ $\begin{array}{lllll}P 899 & 42 & 12.31 & 119 & 1.02\end{array}$

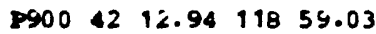
$\begin{array}{llllll}P 901 & 42 & 13.06 \quad 118 & 57.69\end{array}$

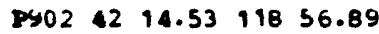
$\begin{array}{lllll}8903 \quad 42 & 15.33 \quad 118 & 56.33\end{array}$ $\begin{array}{lllll}1404 & 42 \quad 15.95 & 118 & 59.13\end{array}$

$P 9542 \quad 16.91 \quad 11856.39$ $\begin{array}{lllll}906 & 42 & 17.78 & 118 & 55.92\end{array}$

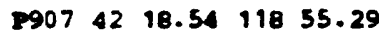
\begin{tabular}{llll}
\hline 408 & 19.29 & 118 & 54.53
\end{tabular}

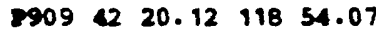

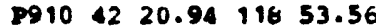
$\begin{array}{llll}2911 & 421.86 \quad 118 \quad 53.86\end{array}$ $\begin{array}{llllll}P 912 & 42 & 22.31 & 118 & 56.67\end{array}$ $\begin{array}{llllll}0913 \quad 22 & 22.61 \quad 118 & 52.73\end{array}$ $\begin{array}{ll}914 & 21.35 \quad 11850.72\end{array}$ mev cos Grav FLET mGAL

$7853.0 \quad 979703.93$ 7878.0979699 .01 $7112.0 \quad 979753.74$ $7177.0 \quad 979735.50$ 7621.0979706 .95 7412.0979729 .52 $7126.0 \quad 979750.50$ 5988.0979837 .64 $4812.0 \quad 979892.78$ 4191.0979950 .33

4243.5979959 .83 $5124.0 \quad 979919.60$ 5609.4979884 .76 $5045.0 \quad 979920.14$ $4975.0 \quad 979923.01$ $5897.0 \quad 979860.73$ $6147.0 \quad 979841.27$ $6125.0 \quad 979839.45$ $5570.0 \quad 979870.86$ $4515.0 \quad 979896.64$

$4563.0 \quad 979893.71$ $4784.0 \quad 979886.45$ $5010.0 \quad 979872.44$ $5474.0 \quad 979846.66$ $5605.0 \quad 979843.31$ 5571.0979848 .73 5530.1979844 .16 5468.1979851 .01 5450.7979853 .67 5410.2979860 .02

$5342.0 \quad 979866.24$ $5323.0 \quad 979868.32$ 5198.6979878 .60 4998.6979891 .50 5063.4979890 .78 4823.6979907 .28 4720.4979914 .20 4706.0979917 .83 4688.9979919 .87 4832.0979913 .38

4675.3979923 .50 $4589.0 \quad 979929.25$ 4569.9979932 .66 4539.6979932 .73 4601.8979931 .28 4606.6979932 .97 $4548.0 \quad 979933.56$ 4534.0979935 .77 4580.4979936 .92 $4846.0 \quad 979927.73$
FREE TERKAIN BOUG AIR WAND COMP ANGM

$\begin{array}{rlllrl}90.1 & 1.0 f & 8.4 & -170.8 & -6.2 & 6744 \\ 87.5 & 0.7 f & 8.3 & -174.4 & -10.0 & 6744 \\ 67.4 & 1.2 f & 6.1 & -170.6 & -6.4 & 6744 \\ 61.7 & 0.61 & 5.5 & -179.2 & -14.5 & 6754 \\ 73.3 & 0.91 & 9.2 & -178.9 & -14.6 & 6744 \\ 73.2 & 0.91 & 7.7 & -173.4 & -9.2 & 6744 \\ 65.1 & 0.6 f & 6.7 & -172.8 & -9.0 & 6744 \\ 43.5 & 0.4 f & 2.3 & -159.9 & 3.8 & 6744 \\ -12.5 & 0.2 f & 1.4 & -176.7 & -13.1 & 6844 \\ -24.0 & 0.0 f & 0.9 & -167.3 & -5.1 & 6744\end{array}$

$\begin{array}{llll}-13.1 & 0.1 f \quad 1.0 & -158.1\end{array}$

$\begin{array}{lllll}25.7 & 0.3 f & 1.6 & -146.9\end{array}$

$\begin{array}{lllll}35.5 & 0.4 f & 1.9 & -155.4\end{array}$

$\begin{array}{lllll}16.2 & 0.1 f & 1.4 & -155.9\end{array}$

$11.9 \quad 0.0$ f $1.3-157.9$

$\begin{array}{llll}37.5 & 0.3 f & 2.0 & -163.1\end{array}$

$\begin{array}{llll}43.7 & 0.3 f & 2.1 & -165.3\end{array}$

$\begin{array}{lllll}41.8 & 0.66 & 2.3 & -166.3\end{array}$

$\begin{array}{lllll}23.1 & 0.2 f & 1.0 & -167.4\end{array}$

$\begin{array}{llll}-27.7 & 0.15 & 1.3-181.7\end{array}$

$\begin{array}{ll}-27.2 & 0.11\end{array}$

$\begin{array}{lll}-16.5 & 0.5 f\end{array}$

$\begin{array}{lll}-10.7 & 0.2 f\end{array}$

$5.50 .3 f$

$\begin{array}{lll}13.5 & 0.3 f\end{array}$

$\begin{array}{lll}15.9 & 0.08\end{array}$

$6.70 .0 f$

$\begin{array}{ll}5.6 & 0.0 f\end{array}$

$\begin{array}{lll}5.6 & 0.0 f\end{array}$

$6.8 \quad 0.4 f$

$\begin{array}{lll}5.5 & 0.2 f\end{array}$

$4.3 \quad 0.41$

$2.10 .3 f$

$\begin{array}{ll}-4.5 & 0.2 f\end{array}$

0.30 .18

$\begin{array}{lll}-6.7 & 0.11\end{array}$

$\begin{array}{ll}-10.9 & 0.5 f\end{array}$

$\begin{array}{lll}-9.6 & 0.16\end{array}$

$-10.4 \quad 0.1 f$

$\begin{array}{ll}-4.3 & 0.2 f\end{array}$

$-90.40 .06$

$\begin{array}{lll}-14.0 & 0.08\end{array}$

$\begin{array}{lll}-13.6 & 0.18\end{array}$

$-17.50 .2 f$

$-14.30 .68$

$-13.40 .68$

$\begin{array}{lll}-19.7 & 0.08\end{array}$

$\begin{array}{lll}-19.5 & 0.0 f\end{array}$

$-14.40 .18$

$\begin{array}{lll}3.2 & 0.2 \mathrm{f}\end{array}$
3.8 B123

12.66734

$5.9 \times 434$

5.70444

3.80454

$-1.56744$

$-3.46754$

-4.1 G744

$-4.66754$

$-16.10634$

$1.1-183.1$ $1.9-179.1$ $1.9-181.2$ $1.6-181.1$ $1.6-177.6$ $1.0-174.5$ $1.0-182.4$ $0.6-181.8$ $0.6-181.1$ $1.2-178.0$

$0.9-177.2$ $1.1-177.6$ 1. $1-175.6$ $1.1-175.3$ $0.7-173.1$ $1.3-171.3$ $2.0-171.2$ $1.8-169.7$

$1.4-170.3$ $0.5-170.0$

$0.8-170.4$ $1.0-170.9$ $2.8-170.8$ $2.6-170.0$ $2.2-169.7$ $0.8-175.4$ $0.3-175.2$ $1.4-170.6$ $1.8-161.7$ $1.6-169.2$
$-17.46634$ -13.2 G744 -15.3 G744 -15.3 G744 $-11.86644$ $-8.46634$ $-16.0 \times 224$ $-15.9 \times 224$ -15.4 N224 -12.5 N224

$-11.96634$ -12.4 N324 $-10.5 \times 228$ $-10.4 \mathrm{B22}$ -8.5 N224 $-6.9 \times 224$ $-7.2 \times 324$ $-5.9 \times 224$ -6.8 B 124 $-6.46744$

$-7.3 \times 1224$ $-7.9 \times 224$

$-6.4 N 224$ $-8.4 N 224$ -7.8 B124 $-7.610324$ $-13.50624$ $-13.36634$ $-8.9 \times 224$ 0.110324
DATE BASE $H-D-Y$ MAML

81886 DENIO 91886 DENIO B1886 DENIO B1886 DENIO - 1886 DENIO B18B6 DENIU B18B6 DENIO 81886 DENIO 81886 DENIO 82186 LLivio

B2486 DENIO 82186 LENIO 82186 DENIO 82186 DENIU 82186 DENIC 82186 DENIC 82186 DENIO 82186 DENIO 82186 DENIO 82286 DEHIU

82286 DENIO 82286 DENIO 82286 DEIIO 82286 DENIO 82286 DENIO 82286 DENIO 82286 DEIIO 82286 DENIIO 82286 DENIO 82286 DENIO

82286 DENIU 82286 DENIC 82286 DENIO 822B6 DENIO 82286 DENIO 82286 DENIO 82286 UENIO 02286 DENIO 82286 DENIO 82286 DENIO

82286 DDNIO 82286 DENIIO 82286 DENIO 82286 DENIU 02286 DENIO 82286 UENIO B2286 DENIO 02286 DENIIO $\$ 2286$ DENIO B2286 DENIO 
rable 7--(continued)

GTATION LATITUDE LONGITUDE NAML DEG MIN DUE MIN

$\begin{array}{lllll}\text { P9 } 15 & 12 & 17.26 & 118 & 41.98\end{array}$ $\begin{array}{llllll}P 916 & 42 & 3.36 & 119 & 29.10\end{array}$

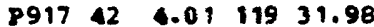
$8918 \varangle 2 \quad 5.5411931 .64$ $\begin{array}{lllll}P 919 & 42 \quad 6.69 \quad 119 & 31.63\end{array}$ $\begin{array}{lllll}P 920 & 42 & 10.86 & 119 & 31.96\end{array}$ $\begin{array}{lllll}P 921 \quad 42 \quad 13.34 \quad 119 & 29.86\end{array}$ $\begin{array}{llllll}P 922 & 42 & 12.96 & 119 & 31.39\end{array}$ $P 923 \quad 42 \quad 13.82 \quad 119 \quad 31.16$ P924 $42 \quad 14.47 \quad 11932.38$

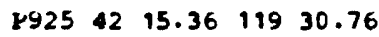

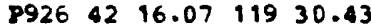
$\begin{array}{lllll}\text { P927 } 42 \quad 17.00 \quad 119 & 30.31\end{array}$ $\begin{array}{lllll}P 928 & 42 \quad 18.73 \quad 119 & 30.55\end{array}$ $8929 \quad 42 \quad 17.33 \quad 11931.40$ $\begin{array}{lllll}\text { P930 } 42 \quad 17.19 & 119 & 32.39\end{array}$ $\begin{array}{llllll}\text { P931 } 42 \quad 17.71 & 119 & 33.25\end{array}$

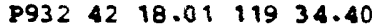
$\begin{array}{lllll}P 933 & 42 & 18.05 & 119 & 35.38\end{array}$ $\begin{array}{lll}P 934 & 42 \quad 17.85 \quad 119 \quad 36.37\end{array}$

$\begin{array}{lllll}P 935 & 42 & 16.80 & 119 & 37.07\end{array}$ $\begin{array}{lllll}8936 & 42 \quad 16.94 & 119 & 38.79\end{array}$ $\begin{array}{llllll}P 937 & 42 \quad 15.70 \quad 119 & 36.42\end{array}$ $\begin{array}{lllll}P 938 & 42 & 18.54 & 118 & 47.33\end{array}$ $\begin{array}{llllll}P 939 & 42 & 16.96 & 118 & 47.87\end{array}$ $\begin{array}{lllll}P 940 & 42 & 16.49 & 118 & 51.91\end{array}$ $\begin{array}{llllll}P 941 & 42 \quad 13.84 \quad 118 & 53.40\end{array}$

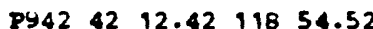

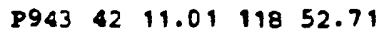
$8944 \quad 42 \quad 16.47 \quad 11850.24$

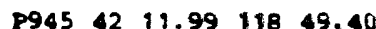
$\begin{array}{llllll}P 946 \quad 42 \quad 9.79 & 118 & 54.53\end{array}$ $\begin{array}{llllll}8947 & 42 \quad 8.71 & 118 & 55.79\end{array}$ $\begin{array}{lllll}\text { PY } 48 & 42 \quad 7.76 \quad 118 & 57.39\end{array}$ $\begin{array}{lllll}8949 \quad 42 \quad 6.52 \quad 118 & 58.51\end{array}$ $\begin{array}{lllll}P 950 & 42 & 10.79 & 118 & 51.31\end{array}$ $\begin{array}{lllll}\text { P951 } & 42 & 12.33 & 119 & 59.67\end{array}$ $8952 \quad 42 \quad 12.86 \quad 119 \quad 58.25$ $\begin{array}{lllll}P 953 \quad 42 & 13.56 & 119 & 57.24\end{array}$ $P 954 \quad 42 \quad 14.30 \quad 119 \quad 56.24$

$P 95542 \quad 14.89 \quad 11954.80$ $\begin{array}{lllll}P 956 & 42 & 16.25 & 119 & 54.21\end{array}$ $\begin{array}{llllll}P 957 & 42 & 16.22 \quad 119 & 56.06\end{array}$ $\begin{array}{lllll}P 958 & 42 & 18.85 & 119 & 59.91\end{array}$ $\begin{array}{llllll}P 959 & 42 \quad 17.69 \quad 120 & 0.45\end{array}$ $\begin{array}{lllll}2960 & 42 & 6.48 & 119 & 44.80\end{array}$ $\begin{array}{lllll}296142 & 5.83 \quad 11940.01\end{array}$ $\begin{array}{lllll}2962 \quad 12 & 2.00 & 119 & 18.63\end{array}$ $\begin{array}{lllll}9963 \quad 12 & 2.80 & 119 & 16.38\end{array}$ $964 \quad 42 \quad 4.49 \quad 119 \quad 13.61$
ELV OBS GRAV
FEET

$4520.0 \quad 979955.59$ 5218.0979852 .60 $5220.0 \quad 979857.77$ 5204.0979863 .04 5194.4979867 .82 5188.0979878 .28 5203.3979881 .06 5196.7979881 .10 5201.6979883 .45 $5325.0 \quad 979882.09$

5218.2979886 .16 5207.2979886 .90 5212.7979887 .59 $5281.0 \quad 979887.31$ 5517.1979870 .86 $5596.6 \quad 979864.99$ 5677.9979862 .71 5771.9979860 .17 5770.0979862 .13 5919.9979851 .32

5728.9979860 .59 5751.6979858 .56 $5774.0 \quad 979854.97$ $5744.0 \quad 979873.01$ 5470.0979881 .51 $5907.0 \quad 979850.89$ $5464.0 \quad 979870.48$ $5346.0 \quad 979875.50$ $5174.0 \quad 979879.23$ $5022.0 \quad 979887.73$

$4995.0 \quad 979896.47$ $5382.0 \quad 979860.58$ 5510.0979851 .46 $5927.0 \quad 979826.22$ $6477.0 \quad 979791.36$ 5103.0979884 .10 $5630.0 \quad 979849.09$ $5875.0 \quad 979835.99$ 6063.0979828 .32 $6265.0 \quad 979817.44$

$6625.0 \quad 979794.13$ $6670.0 \quad 979792.30$ 6076.0979836 .57 $5634.0 \quad 979867.78$ 5670.0979864 .08 $4753.0 \quad 979898.95$ 6130.7979804 .89 5867.0979813 .88 5858.0979813 .68 6096.0979804 .10
TREE TLRRAIN BOUG AIR HAND COMP WNOH

\begin{tabular}{|c|c|c|c|c|c|c|c|}
\hline 6.6 & & .2 & -147.8 & 14.0 & 6744 & 82286 & DENIO \\
\hline 9.9 & $.3 f$ & .8 & -187.6 & -19.2 & 6634 & 82386 & DENIO \\
\hline . & $.0 f$ & 0.4 & -184.6 & -16.6 & 6634 & 82386 & DDNIO \\
\hline 1.0 & $.0 t$ & .3 & -182.6 & -14.9 & 6634 & 2386 & DENIO \\
\hline 1.9 & $.0 f$ & .3 & -180.2 & -12.8 & B124 & 82386 & DENIO \\
\hline 1.7 & & & -97 & & & & DENIO \\
\hline 2.2 & 0.0 & 0.3 & -17 & & & 2386 & DENIO \\
\hline 2.2 & & 0.4 & -17 & & & 1386 & DENIO \\
\hline 3.7 & & .6 & & & & & DENIO \\
\hline 12.9 & & 0.7 & & & & & \\
\hline 5.6 & & .4 & 3.4 & -8.3 & E124 & 1386 & DENIO \\
\hline 4.3 & $.5 f$ & 0.9 & -173 & -9.1 & N224 & 2386 & DENIO \\
\hline 4.1 & $.0 E$ & .4 & 74.7 & -10.2 & N224 & 2386 & DENIO \\
\hline 7.6 & & .5 & -173.5 & -9.5 & 6634 & 2386 & DEIIIC \\
\hline 15,5 & If & 0.6 & 73.6 & -9.3 & B 124 & 2386 & DEIIIO \\
\hline .3 & $.3 f$ & 0.8 & 74.3 & -9.9 & E124 & 2386 & DENIO \\
\hline 1.9 & . $1 f$ & 0.6 & -172.7 & -8.5 & No 24 & 82386 & DENIO \\
\hline .7 & & 0.5 & & -6.1 & B124 & 2386 & DENIO \\
\hline .4 & & 0.5 & & -4.4 & N224 & 386 & DENIO \\
\hline .0 & f & .8 & & & & & ENIC \\
\hline .9 & & .5 & & -6.2 & & & DENIO \\
\hline 5.8 & 0 . & 0.5 & -17 & -7.2 & B124 & 386 & DE:ZIO \\
\hline 26.2 & $0.1 f$ & 0.6 & -17 & -7.1 & 6634 & 82386 & DENIO \\
\hline 37.1 & $0.3 f$ & 1.6 & -15 & 3.0 & C644 & 82486 & DENIO \\
\hline 22.3 & $0.1 f$ & 1.2 & -16 & -2.4 & 6644 & 2486 & Elito \\
\hline 33.4 & $0.2 f$ & 1.5 & -168.1 & -5.6 & G644 & 82486 & DENIO \\
\hline 15.3 & $0.3 \mathrm{f}$ & 0.8 & -171.6 & -8.4 & G654 & 82486 & DENIO \\
\hline 11.4 & $0.3 f$ & 0.7 & -171.6 & -7.8 & G644 & 82486 & DENIO \\
\hline 1.1 & $0.2 f$ & 0.7 & -176.1 & -12.1 & 0633 & 82486 & DENIO \\
\hline-3.9 & 0. & 0.6 & & & G644 & 2486 & DENIC \\
\hline 0.0 & & 0.9 & & .4 & & & NIC \\
\hline 3.8 & 0 & 0.5 & -18 & -16.4 & G644 & & DENIC \\
\hline 8.4 & & 0.8 & -18 & -15.6 & 6634 & 186 & DDNIC \\
\hline 3.7 & & 1.5 & & -13.6 & 6634 & 186 & DENIC \\
\hline 12.4 & & 2.7 & & -12. & 6634 & & ENIO \\
\hline 0.4 & & 0.7 & & & & & NIC \\
\hline 1.8 & & 0.9 & & & & & NIC \\
\hline 1.0 & & 1.0 & & & & & NIC \\
\hline .9 & & .4 & & & & & \\
\hline & & & & -12 & G754 & & \\
\hline 6.5 & & 4.2 & & & & & DENIU \\
\hline 66.9 & & 6.3 & -17 & -12 & G754 & 82586 & DENIO \\
\hline 2.4 & & 1.8 & -17 & -11. & G754 & 82586 & DENIO \\
\hline 1.1 & & 0. & -17 & -9 & 6654 & 82586 & DENIO \\
\hline ?. & 0.08 & 0. & -171.8 & -8.6 & 0644 & 82586 & DDNIC \\
\hline & 0.41 & 2. & -17 & -6.4 & $\mathbf{m 3 4}$ & 82586 & DENIC \\
\hline 4. & $0.2 f$ & & $-184 \cdot 8$ & -18.2 & B124 & 02586 & DLNIC \\
\hline 4. & $0.0 f$ & & -18 & -18.2 & 2644 & 82686 & DENIC \\
\hline & & & -18 & & 0634 & 82686 & DNIC \\
\hline & & & & & 40 & & \\
\hline
\end{tabular}

ISOS ACC DATE BASE WOH CODE $n-D-Y$ NAME

$14.0 \mathrm{G4} 82286 \mathrm{DENIO}$ $-14.96634 \quad 82386$ DENIO -10.9 B124 -10.4 B123 82386 DENIO -9.1 N324 82386 DDNIO -8.35124 B2386 DENIO 6 DENIO -9.3 B124 B2386 DEIIO -9.9 E124 B2386 DENIO B2386 DENIO

82386 DENIO 82486 DENIO 82486 DENIO B2486 DENIO ( 
Table 7-(continued)

STATION LATITUDE LONGITUDE WAME DLG MIN DLG MIN

$\begin{array}{lllll}P 965 \quad 42 & 5.46 & 119 & 11.32\end{array}$ $\begin{array}{lllll}P 966 & 42 & 5.76 & 119 & 9.61\end{array}$ $\begin{array}{lllll}P 967 & 42 & 4.79 & 119 & 8.42\end{array}$ $\begin{array}{lllll}P 968 & 42 & 4.27 & 119 & 4.74\end{array}$ $\begin{array}{lllll}P 969 & 42 & 4.47 \quad 119 & 7.11\end{array}$ $\begin{array}{lllll}P 970 & 42 & 2.49 & 119 & 7.84\end{array}$ $\begin{array}{llllll}P 971 & 42 & 7.36 & 119 & 7.76\end{array}$ $\begin{array}{lllll}P 972 & 42 & 8.65 & 119 & 9.11\end{array}$ $\begin{array}{lllll}P 973 \quad 42 & 9.23 \quad 119 & 10.03\end{array}$ $\begin{array}{lllll}P 974 & 42 & 9.93 & 119 & 10.99\end{array}$

$\begin{array}{lllll}P 975 & 42 & 10.83 \quad 119 & 12.09\end{array}$ $\begin{array}{lllll}P 976 \quad 42 & 12.70 & 119 & 10.76\end{array}$ $\begin{array}{llllll}P 977 & 42 & 15.11 & 119 & 11.33\end{array}$ $\begin{array}{lllll}P 978 & 42 & 10.78 & 119 & 11.82\end{array}$ $\begin{array}{llllll}P 979 & 42 & 19.36 & 119 & 12.47\end{array}$ $\begin{array}{llllll}P 980 & 42 & 21.01 & 119 & 13.07\end{array}$ $\begin{array}{lllll}P 981 & 42 & 22.67 & 119 & 14.41\end{array}$ $\begin{array}{lllll}P 982 & 42 & 25.20 & 119 & 14.84\end{array}$ $\begin{array}{lllll}P 983 \quad 42 & 27.22 & 119 & 15.41\end{array}$

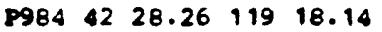

$\begin{array}{lllll}P 985 & 42 & 10.75 & 119 & 14.69\end{array}$ $\begin{array}{llllll}\text { P986 } & 42 & 9.98 & 119 & 15.98\end{array}$ $\begin{array}{lllll}P 987 & 42 & 7.69 & 119 & 14.15\end{array}$ $\begin{array}{llllll}P 988 & 42 & 11.06 & 119 & 16.93\end{array}$ $P 989 \varangle 2 \quad 12.67 \quad 119 \quad 18.42$ $\begin{array}{llllll}P 990 \quad 42 & 14.46 & 119 & 18.52\end{array}$ $\begin{array}{lllll}P 991 & \triangle 2 & 16.05 & 119 & 18.26\end{array}$ $\begin{array}{lllll}P 992 & 42 & 18.21 & 119 & 15.90\end{array}$ $\begin{array}{lllll}P 993 & 42 & 17.82 & 119 & 17.75\end{array}$ $\begin{array}{lllll}8994 & 42 & 9.23 & 119 & 28.30\end{array}$

$\begin{array}{lllll}P 995 & 42 & 10.60 & 119 & 26.78\end{array}$ $\begin{array}{llll}P 996 \quad 42 & 11.56 \quad 119 & 26.53\end{array}$ $\begin{array}{lllll}P 997 \quad 42 & 13.02 \quad 119 & 26.16\end{array}$ $\begin{array}{lllll}P 998 & 42 & 13.72 & 119 & 25.97\end{array}$ $P 999 \quad 42 \quad 16.65 \quad 119 \quad 25.42$

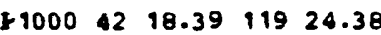
$\begin{array}{llll}P 1001 \quad 19.62 \quad 119 & 24.55\end{array}$ $P 1002 \quad 42 \quad 20.45 \quad 11924.58$ $\begin{array}{lllll}P 1003 \quad 42 & 6.72 \quad 119 & 27.79\end{array}$ PH $143 \quad 25.98 \quad 117 \quad 16.64$

PH $2 \quad 43 \quad 25.38 \quad 117 \quad 18.32$ $\begin{array}{llllll}3 & 43 & 26.76 & 117 & 17.95\end{array}$ PH $43 \quad 28.68 \quad 117 \quad 16.68$ PH $5 \quad 43 \quad 21.16 \quad 117 \quad 16.63$ PH $\begin{array}{llllll}6 & 43 & 20.17 & 117 & 17.68\end{array}$ PH $7 \quad 43 \quad 19.74 \quad 117 \quad 15.72$ $\begin{array}{lllllll}\text { PH } & 43 & 16.61 & 117 & 18.21\end{array}$ P4 $9 \quad 43 \quad 15.43 \quad 117 \quad 16.70$ $\begin{array}{llllll}2410 & 43 & 13.02 & 117 & 15.06\end{array}$ $\begin{array}{llllll}\text { Put1 } & 13 & 16.48 & 117 & 12.57\end{array}$
ELEV Cos Grav PET
PREE TERRAIN DOUG I8OS MCC
AIR DATE BASE

6185.0979798 .71 $6364.0 \quad 979785.78$ $6294.0 \quad 979789.60$ 6493.0979782 .93 6185.0979798 .48 5615.0979830 .05 5955.5979816 .50 $5640.0 \quad 979835.89$ 5553.2979843 .19 5498.7979849 .28

$5452.0 \quad 979854.55$ $5230.0 \quad 979873.40$ $5154.0 \quad 979886.39$ 5145.0979893 .37 $5422.0 \quad 979881.68$ $5386.0 \quad 979888.42$ 5371.0979893 .86 $5085.0 \quad 979918.09$ 4941.0979931 .16 4841.5979936 .37

5618.3979846 .03 $5565.0 \quad 979850.31$ 5918.0979822 .92 5683.7979845 .75 5804.3979842 .21 5612.6979856 .98 $5462.0 \quad 979873.03$ $5424.0 \quad 979878.28$ 5691.0979862 .08 $5230.0 \quad 979869.56$

$5330.0 \quad 979868.00$ 5316.0979873 .82 $5302.0 \quad 979878.13$ 5259.0979882 .53 5356.9979878 .86 5535.0979872 .35 5584.5979870 .85 5488.4979879 .62 5233.0979860 .51 3789.0980111 .68

3598.0980124 .80 4211.0980085 .97 4286.0980088 .32 3651.0980105 .89 3710.0980104 .23 4629.0980039 .37 418.0980060 .92 5289.0980011 .57 6010.0979965 .57 5152.0980023 .90

$23.9 \quad 0.18 \quad 0.9-187.6 \quad-20.6 \quad 654 \quad 2686$ $27.4 \quad 0.16 \quad 1.3-189.9 \quad-23.16754 \quad 82686$ DENIO $26.0 \quad 0.1 f \quad 1.2-188.9 \quad-22.1$ G $\quad$ G4 82686 DENIO

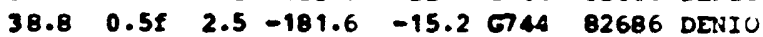
$25.2 \quad 0.1 f \quad 1.1-186.2 \quad-19.4664482686$ DENIO

$\begin{array}{llllllll}6.1 & 0.06 & 0.7 & -186.1 & -18.6 & 654 & 82686 & \text { DENIO }\end{array}$

$\begin{array}{llllllll}17.3 & 0.2 f & 1.4 & -186.0 & -19.7 & \text { N224 } 82686 \text { DENIU }\end{array}$

$\begin{array}{llllllll}5.1 & 0.1 f & 1.0 & -187.7 & -21.5 & \text { N224 } & 82686 & \text { DENIO }\end{array}$

3.40. if $0.9-186.6 \quad-20.5 \times 224 \quad 82686$ DENIO

$\begin{array}{lllllll}3.3 & 0.1 f & 0.7 & -185.0 & -18.9 & \text { N224 } & 82686 \text { DENIO }\end{array}$

$2.8 \quad 0.16 \quad 0.6-184.0 \quad-18.1 \quad 6633 \quad 82686$ DENIO

$\begin{array}{llllllll}-2.0 & 0.06 & 0.3 & -181.5 & -16.1 & 0744 & 82686 & \text { DENIC }\end{array}$

$\begin{array}{llllllllll}0.2 & 0.06 & 0.2 & -176.8 & -12.1 & 6654 & B 2686 & \text { DENIO }\end{array}$

$\begin{array}{llllllll}3.8 & 0.0 f & 0.3 & -172.8 & -8.7 & 6654 & 82686 & D E 7 I I O\end{array}$

$\begin{array}{lllllllll}14.3 & 0.1 f & 0.5 & -171.5 & -8.4 & 6644 & 82686 & \text { DENIO }\end{array}$

$\begin{array}{llllllll}15.2 & 0.1 f & 0.5 & -169.4 & -6.8 & 6644 & 82686 & D E N I O\end{array}$

16.7. $0.1 f \quad 0.8-167.2 \quad-5.0 \quad 6534 \quad 82686$ DEINIO

$\begin{array}{llllllll}10.3 & 0.08 & 0.5 & -164.1 & -2.8 & 6544 & 82686 & \text { DENIO }\end{array}$

$\begin{array}{llllllllll}6.8 & 0.06 & 0.3 & -162.9 & -2.2 & G 734 & 82686 & \text { DENIO }\end{array}$

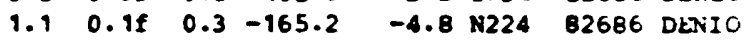

$10.0 \quad 0.1 f \quad 0.5-182.5 \quad-16.5$ N224 82686 DENIT

$\begin{array}{lllllllll}10.5 & 0.1 f & 0.6 & -180.2 & -13.8 & 6634 & B 2686 & D E N I O\end{array}$

$19.70 .16 \quad 0.8-182.9 \quad-16.9$ C644 82686 DEIIO

$\begin{array}{llllllll}15.4 & 0.1 f & 0.5 & -179.4 & -13.3 & \text { N224 } & 82786 & \text { DENIO }\end{array}$

$20.8 \quad 0.1 f \quad 0.6-178.0 \quad-12.4$ B $124 \quad$ B2786 DENIO

$\begin{array}{lllllllll}14.9 & 0.1 f & 0.6 & -177.4 & -12.3 & \text { N224 } & 82786 & \text { DENIO }\end{array}$

$14.4 \quad 0.1 f \quad 0.5-172.8 \quad-8.3 \quad 2733 \quad 82786$ DENIO

$\begin{array}{llllllll}12.8 & 0.1 f & 0.9 & -172.8 & -9.0 & 6734 & 82786 & \text { DENIU }\end{array}$

$22.3 \quad 0.1 f \quad 0.9-172.4 \quad-8.5$ GTS4 82786 DENID

$\begin{array}{llllllll}-0.6 & 0.15 & 0.4 & -180.1 & -13.3 & 6744 & 82786 & \text { DENIU }\end{array}$

$\begin{array}{lllllllll}5.1 & 0.05 & 0.3 & -177.8 & -11.4 & 0644 & 82786 & \text { DENIO }\end{array}$

$8.2 \quad 0.06 \quad 0.3 \quad-174.3 \quad-8.1 \quad 6634 \quad 82786$ DENIO

$\begin{array}{llllllllll}9.0 & 0.06 & 0.3 & -173.0 & -7.2 & 6644 & 82786 & \text { DENIO }\end{array}$

$8.3 \quad 0.0 f \quad 0.3 \quad-172.2 \quad-6.7 \quad 6634 \quad 82786$ DENIO

$9.40 .05 \quad 0.4-174.4 \quad-9.8$ N224 82786 DENIO

$17.1 \quad 0.1 f \quad 0.6-172.6 \quad-8.6 \quad 6634 \quad 82786$ DENIO

$18.4 \quad 0.15 \quad 0.6-172.9 \quad-9.4$ M224 82786 DEIIO

$\begin{array}{lllllllll}16.9 & 0.06 & 0.5 & -171.3 & -8.0 & 6124 & 82786 & \text { DENIO }\end{array}$

$\begin{array}{llllllll}-5.7 & 0.11 & 0.9 & -184.7 & -17.2 & 6644 & 82786 & \text { DENIO }\end{array}$

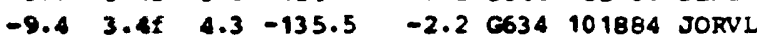

$\begin{array}{rrrrrrrr}-13.3 & 2.2 f & 3.7 & -133.5 & 0.4 & 6634 & 101884 & \text { JORVL } \\ 3.4 & 4.0 f & 7.8 & -133.7 & -0.8 & 6634 & 101884 & \text { JORVL } \\ 9.9 & 1.8 f & 3.7 & -133.9 & -2.5 & 6634 & 101884 & \text { JORVL } \\ -20.9 & 2.5 f & 3.7 & -142.9 & -6.2 & 6644 & 101884 & \text { JORVL } \\ -15.5 & 2.5 f & 4.3 & -139.0 & -1.6 & 6634 & 101884 & \text { JORVL } \\ 6.7 & 2.5 f & 5.3 & -147.3 & -9.9 & 6634 & 101884 & \text { JORVL } \\ 13.1 & 1.2 f & 3.2 & -135.7 & 3.8 & 6644 & 101884 & \text { JORVL } \\ 47.4 & 3.1 f & 6.8 & -127.6 & 12.4 & 6634 & 101884 & \text { JORVL } \\ 72.8 & 1.2 f & 5.1 & -128.6 & 12.8 & 644 & 101884 & \text { JORVL } \\ 45.3 & 1.5 f & 3.8 & -128.1 & 11.3 & 6634 & 101884 & \text { JORVL }\end{array}$


Table 7--(continued)

\begin{tabular}{|c|c|c|c|c|c|c|}
\hline & & & Li & D. & LEV & GRAV \\
\hline NMME & DEG & MIN & LEG & MIN & FEET & MGNL \\
\hline 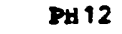 & 43 & 14.52 & 117 & 12.0 & 728.0 & 979982.96 \\
\hline PH13 & 43 & 14.56 & 117 & 9.72 & 5094.0 & 980032.21 \\
\hline P414 & 43 & $14 \cdot 82$ & 117 & 14.46 & 6414.0 & 979932.89 \\
\hline PH15 & 43 & 13.44 & 117 & 17.86 & 5732.0 & 979983.84 \\
\hline PH16 & 43 & 12.05 & 117 & 18.13 & 5396.0 & 980005.56 \\
\hline PH 17 & 43 & 13. & 117 & 21. & 4870.0 & 58002 \\
\hline Pa18 & 43 & 13.88 & 117 & 18.76 & 5100.0 & 98002 \\
\hline 97 & 8) & 13.94 & 117 & 19.37 & 879 & 41.14 \\
\hline & 43 & 15.87 & 117 & 19.85 & 3802.0 & 980104.26 \\
\hline PH21 & 43 & 16.50 & 117 & 16.48 & 4920.0 & 980030.07 \\
\hline
\end{tabular}

trat TERruin DOUG AIK UNND COAP NOM

$61.40 .153 .4-132.0$ $\begin{array}{llll}51.0 & 1.6 f & 3.2 & -120.9\end{array}$ $\begin{array}{lllll}75.3 & 0.3 f & 7.9 & -137.0\end{array}$ $\begin{array}{lllll}64.3 & 0.5 f & 4.7 & -128.0\end{array}$ $\begin{array}{lllll}56.5 & 0.8 f & 3.0 & -126.0\end{array}$ $\begin{array}{lllll}25.1 & 0.7 f & 2.3 & -140.1\end{array}$ $\begin{array}{lllll}46.0 & 0.9 f & 3.6 & -125.7\end{array}$ $\begin{array}{llll}32.6 & 0.7 f & 2.7 & -129.5\end{array}$ $\begin{array}{lllllll}-0.3 & 1.3 f & 3.1 & -128.1\end{array}$ $29.62 .45 \quad 5.5-134.1$
ISOS ACC DATE BASE MHCY CODE M-D-Y MME

0.60644101884 JORVL 20.0 C644 101884 JOKVL 3.2 G754 101884 JORVL 13.16634101884 JORVL $16.0 \quad 674101884$ JORVI 1.06634101884 JORVL 15.2 C754 101884 JORVI 11.46744101884 JOKVL $12.0 \quad 6734101884$ JORVL 5.3 G644 101884 JORVL 
Table 8--Gravity data collected in California.

\begin{tabular}{|c|c|c|c|c|c|c|c|c|c|c|c|c|c|c|}
\hline \multirow{2}{*}{$\begin{array}{l}\text { 8TATIOA. } \\
\text { HAME }\end{array}$} & \multicolumn{2}{|c|}{ LATITUUE } & \multicolumn{2}{|c|}{ LONG I TUDE } & \multirow{2}{*}{$\begin{array}{l}\text { DEV } \\
\text { TEET }\end{array}$} & \multirow{2}{*}{$\begin{array}{l}\text { OBs GRAV } \\
\text { MGNL }\end{array}$} & \multirow{2}{*}{$\begin{array}{l}\text { FREE } \\
\text { AIR }\end{array}$} & \multicolumn{2}{|c|}{ TERRAIN } & \multirow{2}{*}{$\begin{array}{l}\text { Doug } \\
\text { was }\end{array}$} & \multirow{2}{*}{$\begin{array}{l}\text { Isos } \\
\text { war }\end{array}$} & \multirow{2}{*}{$\begin{array}{l}\text { ACC } \\
\text { CODE }\end{array}$} & \multirow{2}{*}{\multicolumn{2}{|c|}{$\begin{array}{ll}\text { DATE } & \text { BASE } \\
\text { H-D-Y }\end{array}$}} \\
\hline & DEC & GIN & LLG & $\boldsymbol{M} \mathbf{N}$ & & & & HAND & $\cos 1$ & & & & & \\
\hline 460 & 40 & 26.51 & 120 & 16.19 & 1472.6 & 9796.51 & 8.7 & 0.31 & 0.7 & -144.5 & 8.4 & B123 & 81985 & \\
\hline 461 & 40 & 29.54 & 120 & 4.46 & 712.6 & 979772.32 & 2.5 & $.0 f$ & 0.6 & -159.0 & -5.0 & $N 123$ & 22385 & CH 23 \\
\hline P477 & 40 & 30.16 & 120 & 30.37 & 849.0 & 979705.56 & 41.7 & $.0 f$ & 6.5 & -152.8 & 0.2 & C644 & 22185 & GERLK \\
\hline P478 & 40 & 30.82 & 120 & 31.26 & 5843.0 & 979710.61 & 45.2 & $.0 f$ & 4.3 & -151.3 & 1.9 & 6644 & 82185 & GERLX \\
\hline 8479 & 40 & 30.93 & 120 & 29.66 & 5449.0 & 979738.97 & 36.3 & 1.18 & 3.1 & -147.8 & 5.6 & C644 & 82185 & GERLK \\
\hline 80 & 40 & 32.29 & 120 & 27.88 & 4551.0 & 979796.82 & 7.8 & . $1 f$ & 1.0 & -147.8 & 6.5 & 6654 & 82185 & GERLX \\
\hline 481 & 40 & 30.53 & 120 & 27.77 & 4535.0 & 979797.82 & .9 & $.0 f$ & 0.6 & -145.5 & 8.2 & G754 & 82185 & GERLK \\
\hline P462 & 40 & 40.27 & 120 & .60 & 5211.0 & 979742.65 & .7 & $5 f$ & 1.2 & -174.2 & -16.0 & 6644 & 82185 & GERLK \\
\hline$P 483$ & 40 & 42.31 & 120 & 39 & 9.0 & 97972 & 4.1 & $2 f$ & 1.0 & 7.0 & 17.9 & 654 & 82185 & GERLK \\
\hline P484 & 40 & 40.73 & 120 & 0.59 & 55.0 & & .7 & . $1 f$ & 4.4 & & -14.4 & 324 & 82185 & GEKLK \\
\hline 8485 & 40 & 39.35 & 120 & 27 & 283.0 & 979741.05 & 0.3 & .91 & 2.1 & -169.2 & -11.6 & 864 & 82185 & GERLK \\
\hline 2495 & 40 & 24.03 & 120 & 11.35 & 5590.0 & 979712.23 & 33.1 & $1.6 f$ & 4.9 & -154.1 & -2.1 & 6644 & 82185 & $\mathrm{CH} 2$ \\
\hline 2496 & 40 & 21.03 & 120 & 8.24 & 6480.0 & 979658.97 & 68.0 & $2.8 f$ & 9.1 & -145.5 & 5.7 & 6644 & 82185 & $\mathrm{CH}$ \\
\hline P497 & 40 & 19.86 & 120 & 6.19 & 6427.0 & 979651.01 & 56.8 & 3.96 & 11.4 & -152.6 & -1.5 & C644 & 82185 & $\mathrm{CH}$ \\
\hline P498 & 40 & 22.54 & 120 & 2.90 & 6923.0 & 979623.05 & 71.4 & 3.08 & 12.1 & -154.1 & $-2 \cdot 8$ & 6644 & 82185 & $\mathrm{CH}$ \\
\hline$P 499$ & 40 & 22.37 & 120 & 1.20 & 6009.0 & 979692.39 & 55.1 & $1.4 f$ & 4.9 & -146.4 & 5.1 & 6644 & 82185 & $\mathrm{CH}$ \\
\hline P508 & 40 & 26.93 & 120 & 9.11 & 5481.0 & 979727.38 & 33.7 & $0.5 f$ & 2.8 & -151.9 & 1.0 & C644 & 82185 & $\mathrm{CH}$ \\
\hline P5 18 & 40 & 27.75 & 120 & 4.68 & 4760.0 & 979776.99 & 14.3 & $0.2 f$ & 0.9 & -148.6 & 4.9 & Q744 & 82385 & $\mathrm{CH}$ \\
\hline P519 & 40 & 26.38 & 120 & 1.19 & 5219.0 & 979748.65 & 31.2 & $0.1 f$ & 0.6 & -147.6 & 5.1 & C643 & 82385 & $\mathrm{CH}$ \\
\hline 24 & 40 & 26.88 & 120 & .78 & 5050.0 & 979758.77 & 24.7 & 0.18 & 0.7 & -148.3 & 4.7 & C744 & 82385 & $\mathrm{CH}$ \\
\hline
\end{tabular}

